\title{
Dust in Supernovae and Supernova Remnants I : Formation Scenarios
}

\section{Sarangi, A.}

2018-04

Sarangi , A , Matsuura, M \& Micelotta , E R 2018 , ' Dust in Supernovae and Supernova

Remnants I : Formation Scenarios ' , Space Science Reviews , vol. 214 , no. 3 , 63 . https://doi.org/10.1007/s11214-

http://hdl.handle.net/10138/234663

https://doi.org/10.1007/s11214-018-0492-7

unspecified

publishedVersion

Downloaded from Helda, University of Helsinki institutional repository.

This is an electronic reprint of the original article.

This reprint may differ from the original in pagination and typographic detail.

Please cite the original version. 


\title{
Dust in Supernovae and Supernova Remnants I: Formation Scenarios
}

\author{
A. Sarangi ${ }^{1,2} \cdot$ M. Matsuura ${ }^{3}$ - E.R. Micelotta ${ }^{4}$
}

Received: 17 June 2017 / Accepted: 17 February 2018 / Published online: 27 March 2018

(C) The Author(s) 2018. This article is published with open access at Springerlink.com

\begin{abstract}
Supernovae are considered as prime sources of dust in space. Observations of local supernovae over the past couple of decades have detected the presence of dust in supernova ejecta. The reddening of the high redshift quasars also indicate the presence of large masses of dust in early galaxies. Considering the top heavy IMF in the early galaxies, supernovae are assumed to be the major contributor to these large amounts of dust. However, the composition and morphology of dust grains formed in a supernova ejecta is yet to be understood with clarity. Moreover, the dust masses inferred from observations in mid-infrared and submillimeter wavelength regimes differ by two orders of magnitude or more. Therefore, the mechanism responsible for the synthesis of molecules and dust in such environments plays a crucial role in studying the evolution of cosmic dust in galaxies. This review summarises our current knowledge of dust formation in supernova ejecta and tries to quantify the role of supernovae as dust producers in a galaxy.
\end{abstract}

Keywords Dust · Supernovae $\cdot$ Chemical kinetics · Infrared $\cdot$ Submillimeter $\cdot$ Presolar grains $\cdot$ SN1987A

Supernovae

Edited by Andrei Bykov, Roger Chevalier, John Raymond, Friedrich-Karl Thielemann, Maurizio Falanga and Rudolf von Steiger

A. Sarangi

arkaprabha.sarangi@nasa.gov

$\bowtie$ M. Matsuura

matsuuram@cardiff.ac.uk

E.R. Micelotta

elisabetta.micelotta@helsinki.fi

1 NASA Goddard Space Flight Center, Greenbelt, MD, USA

2 CRESST II, The Catholic University of America, Washington DC, USA

3 School of Physics and Astronomy, Cardiff University, Cardiff, UK

4 Department of Physics, University of Helsinki, Helsinki, Finland 


\section{Introduction}

The solid grains in space which may vary in size from the dimension of a few molecules to the order of a few microns, are broadly classified as cosmic dust. Cosmic dust can be of diverse chemical types, which includes carbon (amorphous and graphite), silicates (olivines, pyroxenes, both amorphous and crystalline), metal oxides (alumina, fayalite, magnesia, spinel), silica, pure metals (iron, magnesium, silicon), metal sulphides, carbides and some other species yet to be determined with certainty (Molster et al. 2010; Cherchneff 2013). Dust accounts for about 1-2\% of the total baryonic mass in the universe. On the other hand, it is responsible for up to $50 \%$ of the total radiation from some galaxies, which rightfully justifies its importance. These dust grains are efficient in absorbing and scattering UV, optical and near-infrared (IR) radiations, and re-emitting the absorbed energy in the mid-IR and submillimeter (submm) wavelengths (Martin 1978; Li 2005). Owing to their continuum opacities, the dust grains absorb and emit over a large swathe of the electromagnetic spectrum (Draine 2003). Therefore, dust in space profoundly influences the spectra and colours of stars and galaxies. Moreover, cosmic dust acts as the basic building block for the planets in protoplanetary disks around main-sequence (MS) stellar systems (Brownlee 1985; Dwek 2006). The branches of science related to the study of cosmic dust has flourished over last four decades (Grün et al. 2001). It has brought together scientists (astronomers, meteoriticists, physicists, chemists, mineralogists and many more) from various disciplines and expertise to focus on the development of the field.

The earliest discoveries of molecule and dust clouds, leading to interstellar reddening were inferred in the first part of the 20th century (Barnard 1919; Trumpler 1930). However, only after the advent of IR astronomy in the 1960s, we have made a fast progress towards the current state of the art. The infrared region of the electromagnetic spectrum ranges from $700 \mathrm{~nm}$ (the red edge of the visible spectrum) to approximately $1 \mathrm{~mm}$ in wavelengths and is further subdivided into near-IR, mid-IR and far-IR regions. The biggest challenge to the development of IR astronomy, is that only a small part of the IR spectrum can actually be observed from ground-based facilities, owing to the absorption by earth's atmosphere, mainly by water vapour. In order to overcome the impediment of atmospheric shielding, the following schemes have been adopted: (a) to set up telescopes on the top of high altitude mountain peaks, (b) to raise the telescope above the atmosphere by helium balloons, (c) to attach the telescopes in jet transport aeroplanes, and (d) to launch IR satellite telescopes (since 1983) (Walker 2000). Later on, the ground-based telescope facilities with IR capabilities have also been developed. Some of the most important telescopes, operating in the near-IR, mid-IR, far-IR and submm, launched in the 21st century are the Hubble Space Telescope (optical and near-IR), Spitzer space telescope (3-180 $\mu \mathrm{m})$, AKARI (2-200 $\mu \mathrm{m})$, Herschel space observatory (55-672 $\mu \mathrm{m})$ (until 2013) and WISE (3-25 $\mu \mathrm{m})$. The Atacama Large Millimeter/submillimeter Array (ALMA), situated high on the Chajnantor plateau in Chile, is a high resolution millimeter-submillimeter telescope, which can study radiations from the coldest objects in the universe. The James Webb Space Telescope, under preparation for launch in 2018, is designed to boost IR astronomy with its unprecedented resolution and sensitivity in $0.6-27 \mu \mathrm{m}$ range. The energy radiated in the IR is largely thermal in nature. Therefore, IR photometry and spectroscopy have become the crucial tools to study the dust continuum from various sources, vibrational transitions in molecules, signatures from exoplanets and the high redshift universe.

Using the newly developed techniques, confirmed evidence for the presence of cosmic dust has been established through various studies over the past couple of decades. Having said that, it is equally necessary at this point to investigate the potential sources of origin 
Fig. 1 Hertzsprung-Russell diagram showing luminosity-temperature relation of stars at their evolutionary phases as function of main sequence initial mass. The potential stellar sources of cosmic dust are marked with circles. The core-collapse supernova $(\mathrm{SN})$, the final fate of the RSG (red super giant) phase as shown in the diagram, is a major source of dust formation

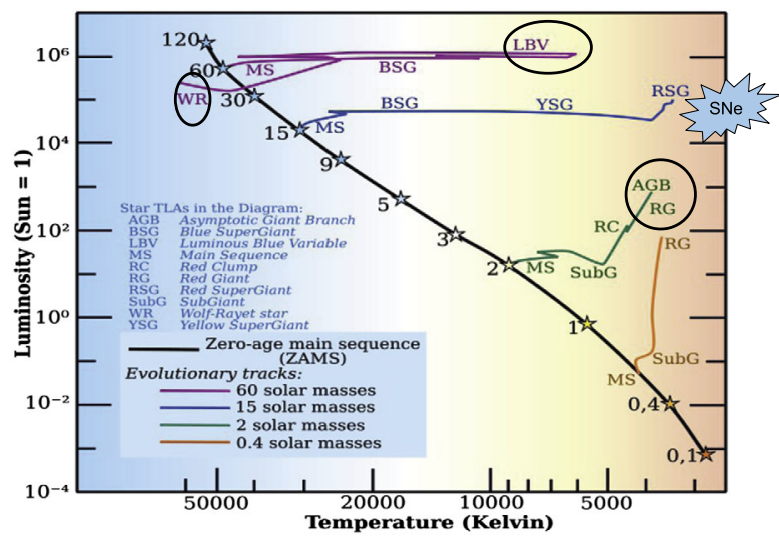

for dust present in space. Cosmic dust is made up of large clusters of chemical compounds. Hence its synthesis requires chemical processes, which are sensitive to the local gas densities and temperatures. The chemical processes that are prevalent at moderately high temperatures $\sim 1000-2000 \mathrm{~K}$ and high gas densities govern the dust formation scenario. Such physical conditions are mainly encountered in evolved stellar outflows or winds and ejecta of novae and supernovae (Cherchneff 2010, 2013; Kozasa et al. 2009). However, the required temperature and densities in these evolving circumstellar environments persist only for a short timescale making the entire mechanism more complex.

The evolved stellar environments responsible for dust formation in galaxies are presented in Fig. 1 with their respective position on the Hertzsprung-Russell diagram. The quiescent mass outflows of the low mass $\left(<8 \mathrm{M}_{\odot}\right)$ stars in their Asymptotic Giant Branch (AGB) phase are thought to be major dust producers in galaxies (Whittet 2003; Cherchneff 2012; Gobrecht et al. 2016). Core-collapse SNe, the ultimate fate of massive (8-30 $\mathrm{M}_{\odot} \mathrm{MS}$ mass) stars, marked in the figure (not in the scale of the H-R diagram), are a prime source for dust formation (Wooden et al. 1993; Sugerman et al. 2006; Dwek 2006; Kozasa et al. 2009; Kotak et al. 2009; Cherchneff 2013; Todini and Ferrara 2001; Szalai et al. 2011). Additionally, periodic mass ejections in massive Luminous Blue Variable (LBV) stars (Hillier et al. 2001; Ferland et al. 2005) and mass-loss events in the Wolf-Rayet stars (Harries et al. 2004) are also associated to dust formation in the galaxies. However, AGB stars and CCSNe are the known to be the main stellar sources. In case of supernova ejecta, where the stellar core has already collapsed, the physical conditions evolve very fast. Therefore, the entire process of dust synthesis occurs in less than a decade. Synthesis of dust in a suitable environment proceeds in two steps: the nucleation and the condensation (Cherchneff and Dwek 2009; Sarangi and Cherchneff 2013). Out of all the potential sources, this chapter aims to discuss and quantify the contribution of supernovae as dust producer in the galaxies.

Dust in dense clouds with a visual extinction exceeding $\sim 3$ mag is characterized by solid cores surrounded by ice mantles. The presence of ice mantles in the dense interstellar clouds compared to the small dust present in the diffuse ISM confirms the growth of grains in the clouds. The grains grow in mass over the lifetime of the molecular clouds. For significant growth of dust grains in the ISM, the necessary conditions are: (a) the presence of preexisting grain cores on which accretion takes place, (b) the accretion timescales must be shorter than the lifetime of the clouds (Dwek and Cherchneff 2011). Interstellar dust grains are subject to various turbulent forces over the span of its lifetime, which either reduce the sizes of the grains or completely destroy them (Dwek 1998; Jones 2004). According to theoretical models, for dust grains to survive in the ISM, a continuous source of newly formed 
Fig. 2 The current classification of supernovae done on the basis of the spectral types and light curves. On the basis of explosion mechanism, supernova explosion categorised as thermonuclear or core-collapse (Turatto 2003)

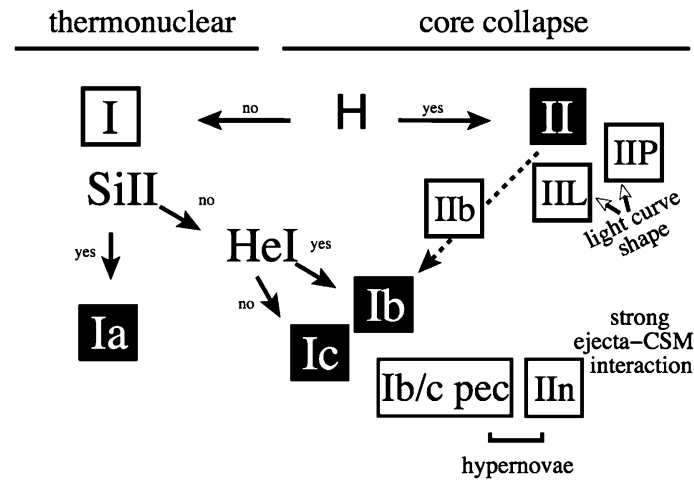

grains must be present in the galaxy which can compensate for the loss due to destruction processes in the clouds (Dwek 2006). Interstellar silicates are predominantly amorphous, whereas stardust silicate samples from meteorites are about $20 \%$ crystalline (Li and Draine 2001; Kemper et al. 2005; Li et al. 2007) in nature. This is consistent with the notion that dust in the ISM might have originated at a different source. Therefore, synthesis of new seeds from the gas phase is highly unlikely in the ISM due to low densities and temperatures.

Even though there remains no ambivalence on supernovae being a prime dust factory in the universe, the mechanism of dust formation still remains unclear. Further, depending on the subclasses of $\mathrm{SNe}$, they are subject to diverse physical conditions directly impacting the chemical processes. SNe are classified broadly on the basis of their optical spectra and some subclasses are defined by the nature of their light curves. The taxonomy is progressively developing since 1941 (Minkowski 1941) and is extensively reviewed by Filippenko (1997) $\&$ Wheeler and Benetti (2000). A schematic presentation of the SNe types is shown in Fig. 2. Here we discuss a few important subclasses of $\mathrm{SNe}$, in light of their role in dust formation.

Pair-instability SNe (PISNe): Based on the explosion mechanism, a large section of massive stars $\left(>100 \mathrm{M}_{\odot}\right)$ are categorised as pair-instability supernova, a case common among Population III and some Population II stars. There is no observational constraint on such objects and neither there is any confirmed evidence for dust formation in the ejecta. However, to account for the dust content in some high-z galaxies, PISNe are considered as prime dust forming agents in those environments. Theoretical studies dealing with nucleosynthesis and hydrodynamics in the pre- and post-explosion massive stars provide the backbone to understand the physical conditions relevant to these systems (Umeda and Nomoto 2002; Heger and Woosley 2002; Fryer et al. 2001). A dust mass of $0.1 \mathrm{M}_{\odot}$ per supernova event has been estimated by Cherchneff and Dwek (2009) adopting a chemical kinetic approach.

Type Ia: Supernovae type Ia is characterised by the absence of hydrogen lines in the spectra. The mechanism of explosion is thermonuclear, which occurs in low mass stars $\left(<8 \mathrm{M}_{\odot}\right.$ ) in binary systems (Branch et al. 1995). Several studies have advocated in support of type Ia's as a probable dust producer (Clayton et al. 1997; Travaglio et al. 1999). Gomez et al. (2012) have investigated two Type Ia remnants, Tycho and Kepler, using Herschel PACS and SPIRE photometry at 70-500 $\mu \mathrm{m}$. Their findings suggest a presence of a warm dust component in the spectra at $\sim 82 \mathrm{~K}$, resulting from a dust mass of $\sim 3.0 \times 10^{-3} \mathrm{M}_{\odot}$. The IR emission features are found to be spatially consistent with the X-ray emitting region and the circumstellar region swept up by the SN blast-wave. There has been no observation till date of new dust formation in the ejecta of Type Ia's (Gomez et al. 2012). On the other hand, theoretical study by Nozawa et al. (2011) has predicted a possibility of new dust formation 
in the ejecta, with a bottom heavy size distribution. However, that being the case, the newly formed dust grains are almost likely to get completely destroyed by the reverse shock in course of their injection into the ISM (Nozawa et al. 2011).

Type Ib/c: These SNe types are characterised by the absence of $\mathrm{H}$ and Si II lines and the presence of He I in case of Type Ib, where as in Type Ic He I lines are also absent (Barbon et al. 1999; Matheson et al. 2000b). These objects are identified as core collapse SNe of massive stars $\left(>25 \mathrm{M}_{\odot}\right.$ ) which have been stripped of their hydrogen envelope. They show evidence of shock interactions with dense circumstellar environments (Chevalier 1982) at early times after explosion. Presence of dust continuum in the spectra of Type Ib SN 1990I was first reported by Elmhamdi et al. (2004). Further, peculiar Type Ib SN2006jc has shown dust signatures as early as day 50 post explosion (Nozawa et al. 2008; Smith et al. 2008; Di Carlo et al. 2008), which originate from the dense shell formed in the post-shock CSM. Mid-IR continuum recorded by Spitzer from Type Ic SN2005at and SN2007gr confirm the presence of dust in the environment (Kankare et al. 2014). The source of the emission can be either an echo or the expanding ejecta, it is yet to be understood with clarity.

Type IIb: Type IIb's are core collapse supernovae which have early time signatures similar to Type II's (presence of prominent $\mathrm{H}$ lines) and a late time spectra similar to Type Ib's (Matheson et al. 2000a). The decline of the lightcurves in Type IIb SNe are much faster compared to other Type II, which implies a smaller mass of the ejecta. Due to the absence of the outer H-envelope after explosion, the ejecta expands faster and the typical gas densities are lower (Nozawa et al. 2010) when compared to a Type IIP. Therefore, Type IIb supernovae do not form dust as efficiently as Type IIP's (Biscaro and Cherchneff 2014, 2016). IR and submm observations of galactic SN remnant Cassiopeia A of Type IIb have revealed the presence of dust ranging between $10^{-4} \mathrm{M}_{\odot}$ (Douvion et al. 2001; Dunne et al. 2003) to $0.08 \mathrm{M}_{\odot}$ (Barlow et al. 2010; Sibthorpe et al. 2010).

Type IIP: The SNe Type II-P (Plateau) is the most abundant type of core-collapse supernovae in the local universe (Barbon et al. 1979). It differs from Type I's by the presence of prominent $\mathrm{H}$ lines in the spectra. The light curve is characterised by a flat plateau type nature during the first few months after explosion. Massive stars in the $8-25 \mathrm{M}_{\odot}$ range can result to Type II-P supernovae at the end of their lives. The presence of a large $\mathrm{H}$-envelope as the outer shell of the ejecta makes the outflowing ejecta massive and slow moving. Moreover, the densities of the expanding He core remain $\sim 10^{2} / 10^{3}$ higher than Type IIb's. Based on the degree of stripping of the envelope, SNe can be arranged in sequence as Type IIP-IILIIb-Ib-Ic (Nomoto et al. 1995) in the ascending order. Also, this is the order of importance in terms of dust synthesis. Therefore, the ejecta of Type IIP SNe is the most efficient and copious source of dust among all SNe types (Szalai and Vinkó 2013; Sarangi and Cherchneff 2015; Cherchneff 2013; Kotak et al. 2009) and our discussion in this chapter will be largely based on Type IIP's.

Type IIn: SNe Type IIn, introduced by Schlegel (1990), are characterised by the presence of narrow $\left(\sim 100 \mathrm{~km} \mathrm{~s}^{-1}\right)$, intermediate $\left(1-4 \times 10^{3} \mathrm{~km} \mathrm{~s}^{-1}\right)$ and broad $\left(10-15 \times 10^{3} \mathrm{~km} \mathrm{~s}^{-1}\right)$ velocity width components. The narrow component originates from the slow moving CSM before being traversed by the supernova shock. The source of intermediate width component is the post-shock region of the CSM, where as the broad component arises from the fast expanding SN ejecta (Smith et al. 2008). Observations indicate early interactions between the ejecta and the circumstellar medium (Smith et al. 2012), which lead to a velocity shear followed by shocks. Many recent studies have reported the presence of warm dust in such environments (Fassia et al. 2001; Mauerhan et al. 2013; Andrews et al. 2011; Smith et al. 2012; Gall et al. 2014). 


\section{Observations of Dust Formation in Core-Collapse Supernovae}

Using modern high-resolution telescopes, a lot of important information can be derived from a single observation and a general trend for dust formation in supernovae can be studied. The formation of warm dust in the ejecta is characterised by the following: (a) an excess in the mid-IR region of the spectral energy distribution due to thermal emission (Roche et al. 1991; Bouchet and Danziger 1993), (b) a sharp decline in the optical light curve (Danziger et al. 1991), (c) a progressive and systematic blue-shift of emission line profiles when the receding part of the ejecta is increasingly blocked by newly formed dust (Lucy et al. 1989), (d) diminishing line emission of elements and molecules compared to the adjacent continuum, owing to depletion in dust (Bouchet and Danziger 1993; Kotak et al. 2009). The first two cases might also arise either due to an IR echo from pre-existing dust in the circumstellar material (Smith et al. 2012) or from the escape of radioactive luminosity due to decrease in optical depth in the ejecta. But the occurrence of three or all of the four phenomena concurrently, clearly indicates the formation of new dust in the ejecta.

There are several local SNe that observers have closely monitored over the past few decades. We shall now discuss the findings from some important $\mathrm{SNe}$ with regard to their history of dust formation and its evolution in the ejecta.

\subsection{Dust Formation in the Ejecta of SN 1987A}

The explosion of a core-collapse SN was detected in the Large Magellanic Cloud, only $50 \mathrm{kpc}$ away on the 23rd of February, 1987 (Kunkel et al. 1987). Since its detection, studies of SN 1987A have led to many discoveries of SNe and SNRs.

\subsubsection{Historical Observations of Dust in SN 1987A}

One of the major discoveries associated with the SN 1987A is the detection of newly formed dust (Danziger et al. 1989). From about 615 days, thermal emission of dust has been detected at near and mid-infrared (IR) with an increase in contrast and a shift towards longer wavelength in time (Fig. 3). The reported dust mass was $10^{-4} \mathrm{M}_{\odot}$ at day 775 with the temperature of 300-400 K. However, the possibility of larger dust mass was suggested because dust could be optically thick (Wooden et al. 1993). At almost the same time ( $\sim 530$ days), the optical light curve declined at a much steeper rate than the predicted light curve accounting for the decay of ${ }^{56} \mathrm{Co}$ and ${ }^{57} \mathrm{Co}$ (Fig. 4: Whitelock et al. 1989). It has been interpreted that, the dust grains that are formed, are causing the extinction in optical wavelengths (Danziger et al. 1989). Furthermore, asymmetric line-profile has also been detected simultaneously. Lucy et al. (1989) modelled the blue-shifted line profile absorbed by the dust that has condensed in the ejecta.

It had been suggested that dust could be present in clumps instead of being smoothly distributed across the ejecta (Lucy et al. 1989; Wooden et al. 1993). If a large mass had been evenly distributed in the ejecta, UV and optical energy would have been totally absorbed resulting in a black out (Hoyle and Wickramasinghe 1970; Dwek 1988). In order to avoid such black out, dust must be present in clumps so that UV and optical light can escape between the clumps. If dust is located in clumps, and if individual clumps are assumed to be optically thick, it is difficult to estimate the actual dust mass. It possibly suggests that the dust masses as large as $0.5 \mathrm{M}_{\odot}$ (Lucy et al. 1989) or $1 \mathrm{M}_{\odot}$ (Dwek 1988). Whether it is feasible to accommodate such a large amount of dust in early days still remains a matter of 

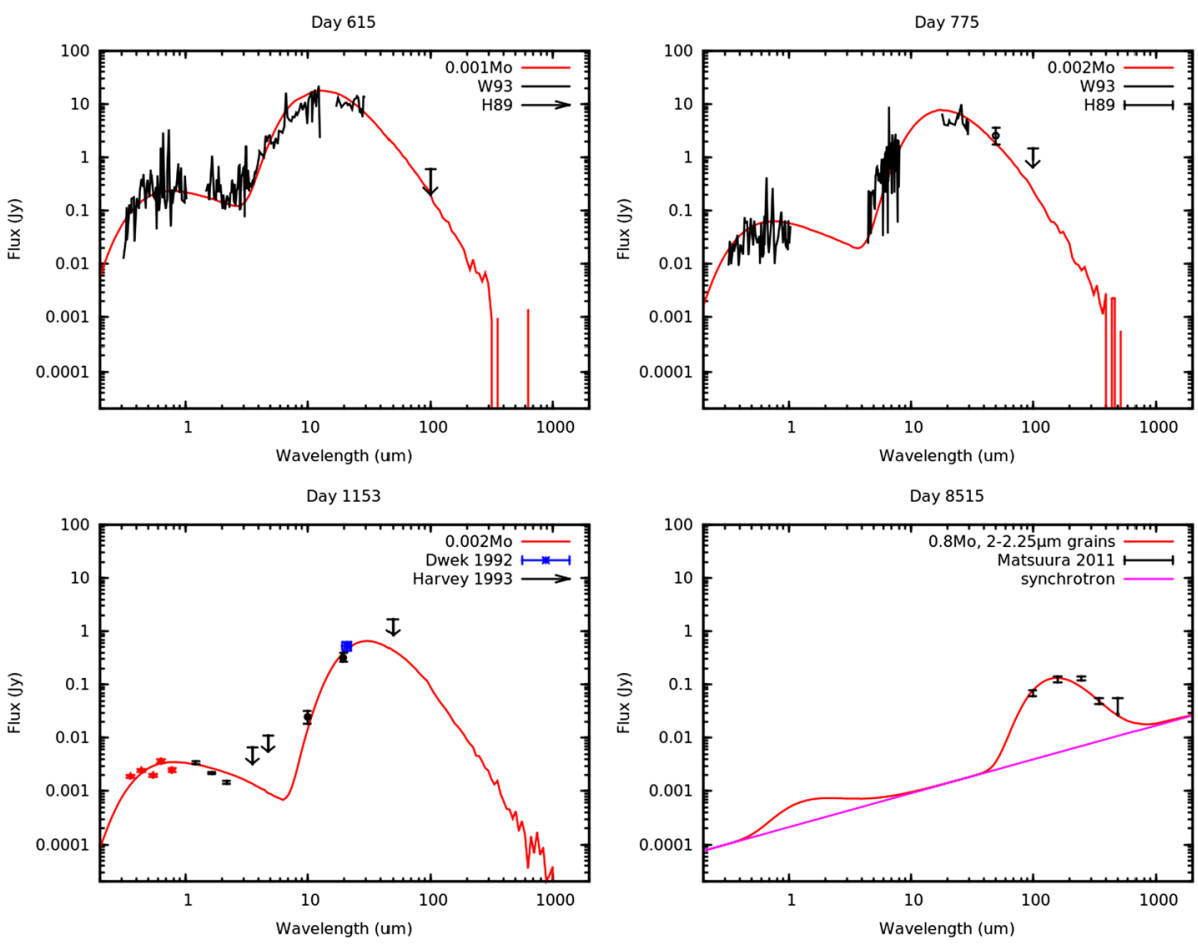

Fig. 3 Time evolution of thermal dust emission of SN 1987A. A broad thermal emission appears at mid-infrared, indicating formation of dust. The peak is shifting towards the longer wavelength in time. The figure is reproduced from Wesson et al. (2014), based on observational data from Wooden et al. (1993), Harvey et al. (1989), Bouchet and Danziger (1993), Dwek et al. (1992), Matsuura et al. (2011)

Fig. 4 The light curve of SN 1987A. The measured luminosity (squares) more sharply declined at about day 530 , than the predicted light-curve from ${ }^{56} \mathrm{Co}$ and ${ }^{57} \mathrm{Co}$ decay. This further decline was attributed to the newly formed dust in the ejecta (Whitelock et al. 1989)

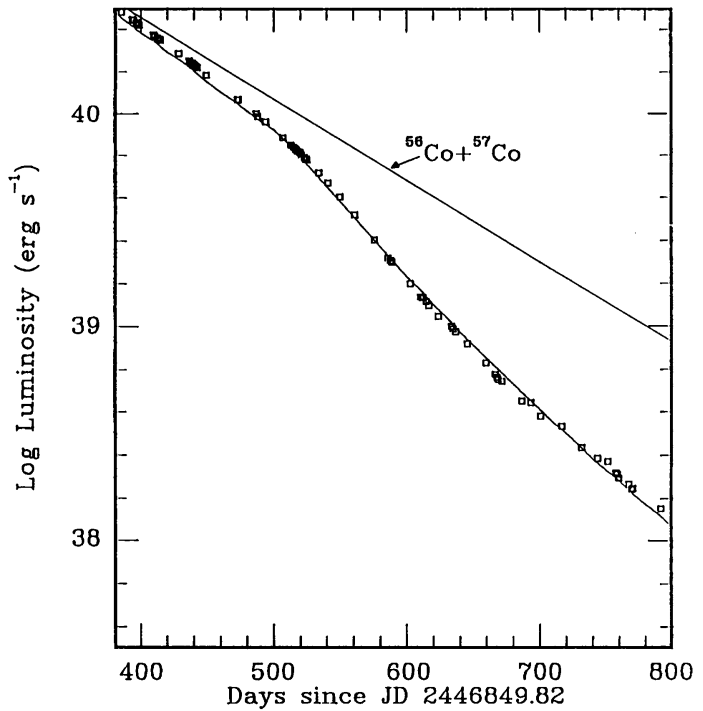


debate (Ercolano et al. 2007; Wesson et al. 2014; Bevan and Barlow 2016; Dwek and Arendt 2015).

Since its detection, thermal emission of dust had been monitored at mid-infrared up to day 1,316 (Suntzeff and Bouchet 1990; Bouchet et al. 1991; Dwek et al. 1992). The following study was at day 6000 with nearly a 13 year gap in between the observations (Bouchet et al. 2004). By day $\sim 1150$, the temperature had dropped to $150 \mathrm{~K}$ with an inferred dust mass of $5 \times 10^{-4} \mathrm{M}_{\odot}$ (Dwek et al. 1992).

Ten years later, mid-infrared observations found dust thermal emission from the circumstellar ring (Bouchet et al. 2006). The ring composed of the material that were expelled from the progenitor star when it was in the red supergiant phase about 20,000 years ago (McCray 1993). The fast $\left(\sim 2000 \mathrm{~km} \mathrm{~s}^{-1}\right)$ blast wave from the explosion has collided into the slowly expanding $\left(\sim 100 \mathrm{~km}^{-1}\right)$ circumstellar ring. The collisions and subsequent shocks yield X-radiation heating dust grains with $300-400 \mathrm{~K}$ radiation at mid-infrared. The infrared luminosity of the ring dust continued to increase concurrently with a constant increase of the X-ray brightness, suggesting destruction of dust grains in the ring (Dwek et al. 2008). However, since day 8,500, infrared brightness started declining, while X-ray luminosity still increased. That is almost the time when the knots around the known ring structure started to brighten up, so that $\mathrm{SN}$ waves appeared to have overtaken the main part of the existing ring (Fransson et al. 2015).

\subsubsection{Detection of a Large Mass of Dust in the Ejecta}

In 2010, the HERSCHEl SPACE ObSERVATORY discovered far-infrared emission towards SN 1987A (Fig. 5: Matsuura et al. 2011). The inferred dust mass was 0.4-0.7 $\mathrm{M}_{\odot}$ with the temperature of $\sim 20-30 \mathrm{~K}$. The only place that can form such a large mass of dust would be the ejecta, where a large mass of refractory elements are present. The confirmation of the presence of dust in the ejecta was made by ALMA, soon after the HERSCHEL observations. ALMA high angular resolution image (angular resolution of $0.3^{\prime \prime}$ ) enabled to resolve SN 1987A system at submillimeter wavelengths, detecting thermal emission from cold dust towards the ejecta (Indebetouw et al. 2014), with a mass of $\sim 0.5 \mathrm{M}_{\odot}$ (Matsuura et al. 2015). Detailed analysis of ALMA data by Zanardo et al. (2014) suggested that there could be an extra component beyond dust at millimetre wavelength, which can be associated with the pulsar winds.

Importantly, the ejecta's expansion velocity of SN 1987A might appear to be too fast for dust formation. Typically dust grains were predicted to be destroyed by SN winds with velocities exceeding $100 \mathrm{~km} \mathrm{~s}^{-1}$ (Jones et al. 1994). On the other hand, the velocities of SN 1987A ejecta are typically $2000 \mathrm{~km} \mathrm{~s}^{-1}$ (full width of half maximum (FWHM); McCray 1993) much larger than the dust destruction velocities. Dust grains can form and survive in such a high velocity environment, because in the rest frame of dust grains, the velocity is much smaller. The expansion velocities reach $2000 \mathrm{~km} \mathrm{~s}^{-1}$ a few months after the explosion, and following that, the velocities were measured to be constant (McCray 1993). Dust grains have formed only after these expansion velocities settled. Therefore currently in SN1987A, the dust grains are expanding at much faster speed without being impacted by shocks.

\subsubsection{Look Back at the Historical Measurements of Dust in SN 1987A}

The detection of a large mass $\left(\sim 0.5 \mathrm{M}_{\odot}\right)$ of ejecta dust at $\sim$ day 8500 reignites the debate if such a large mass of dust is present from early days, starting from day 650 . The inferred 
Fig. 5 Image of SN 1987A and surrounding ISM (Matsuura et al. 2011). Spitzer $8 \mu \mathrm{m}$ in blue,

Spitzer $24 \mu \mathrm{m}$ in red and Herschel $250 \mu \mathrm{m}$ in green. LMC field stars are seen in blue, while SN 1987A, which contain significant mass of cold dust, is seen as a green point source in this image

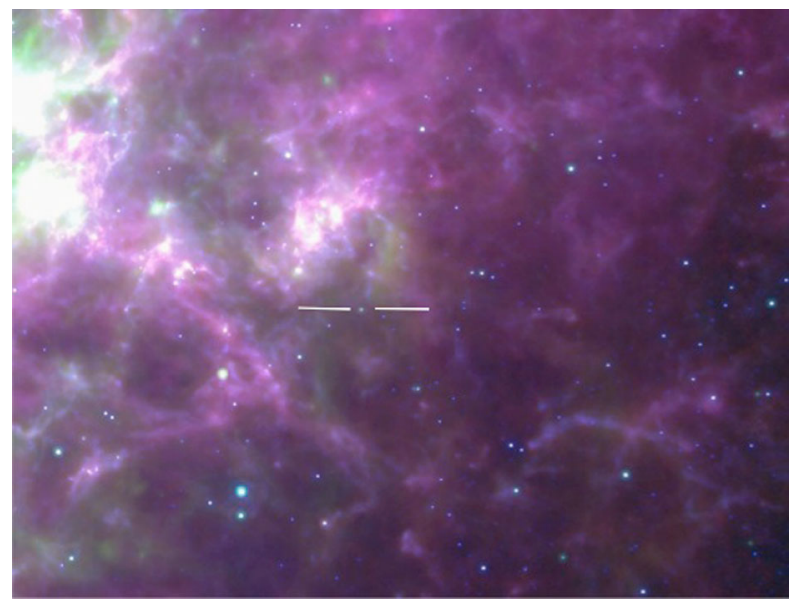

dust mass in early days was $10^{-4} \mathrm{M}_{\odot}$ at day 775 , while measurements at late day $(\sim$ day 10,000) suggests $\sim 0.5 \mathrm{M}_{\odot}$. There are two possibilities that can resolve the disparity. The first possibility is that inferred dust masses in early days were underestimated because the fitting assumed optically thin dust, but the emission was actually optically thick and a large mass of dust was present from the early days itself (Dwek and Arendt 2015). Alternative possibility is that the dust mass has increased over time by growing dust grains (Wesson et al. 2014; Bevan and Barlow 2016). Using currently available observational data, it is not possible to disentangle these two cases.

\subsubsection{Molecules in SN 1987A}

$\mathrm{SN} 1987 \mathrm{~A}$ is also the first $\mathrm{SN}$ where molecule formation was reported. The emission of CO $v=2$ bands at $2.3 \mu \mathrm{m}$ started appearing possibly 112-136 days after the explosion, with much more recognisable features from day 192 (Spyromilio et al. 1988; Catchpole et al. 1988). CO $v=2$ bands at $2.3 \mu \mathrm{m}$ and $v=1$ bands at $4.6 \mu \mathrm{m}$ continued to be detected until about day 600 and then disappeared from infrared spectra (Wooden et al. 1993).

More than 20 years after, $\mathrm{CO}$ was detected at millimetre wavelength after high sensitivity millimetre telescope, the ALMA, started operation in 2011 (Kamenetzky et al. 2013; Matsuura et al. 2017). The analysis of these rotational bands suggests an excitation temperature of $20-50 \mathrm{~K}$, which is much lower than the estimated temperature of $1200-4000 \mathrm{~K}$ in early days (up to day 600; Liu et al. 1992; Liu and Dalgarno 1995). It has been perceived that initially the gas temperature is high enough to excite vibrational-rotational bands. When the gas temperature drops, $\mathrm{CO}$ population is mainly in rotational transitions.

Not only CO but also detections of a few other molecules have been reported in SN 1987A. SiO fundamental bands at 4 and $8 \mu \mathrm{m}$ appeared at about day 200 (Rank et al. 1988; Aitken et al. 1988; Roche et al. 1991; Meikle et al. 1989) and SiO rotational lines at millimetre wavelength were detected at day 9,173 onwards (Kamenetzky et al. 2013; Matsuura et al. 2017). Potential detections of $\mathrm{CO}^{+}, \mathrm{CS}$ and $\mathrm{H}_{3}^{+}$were also reported (Meikle et al. 1989; Miller et al. 1992) though not yet well established (Cherchneff and Dwek 2009). In later epoch, vibrationally excited $\mathrm{H}_{2}$ at $2 \mu \mathrm{m}$ was found since day 6,832 (Fransson et al. 2016), and $\mathrm{HCO}^{+}$and $\mathrm{SO}$ at millimetre wavelength at day 10,037 (Matsuura et al. 2017).

The ALMA offers angular resolution of 50 miliarcsec images of $\mathrm{CO}$ and $\mathrm{SiO}$ in the ejecta (Abellán et al. 2017) as shown in Fig. 6. It is found that the $\mathrm{CO}$ and $\mathrm{SiO}$ clumps in the 
Fig. 6 ALMA CO $J=2-1$ (red) and $\mathrm{SiO} J=5-4$ (green) images overlaid on the HUBBLE SPACE TELESCOPE $\mathrm{H} \alpha$ image of the circumstellar ring of SN1987A. The figure is published by Abellán et al. (2017) and the HST image taken from Larsson et al. (2016)

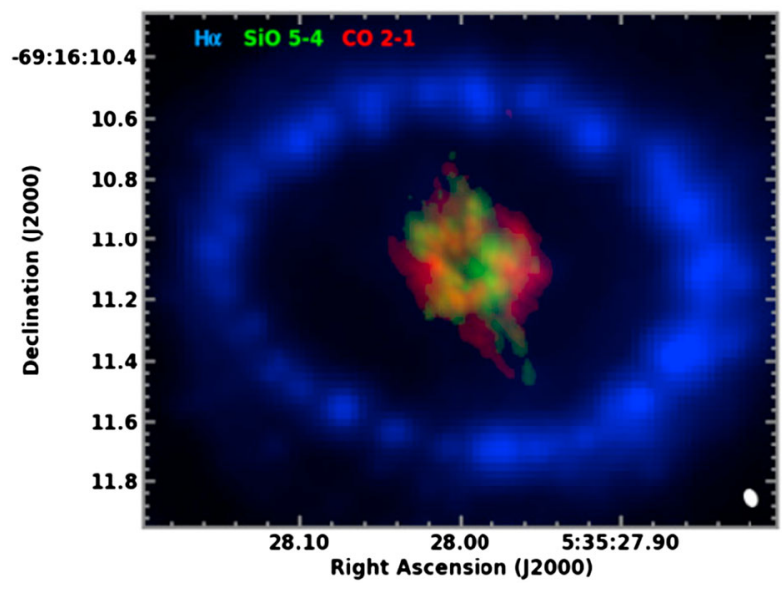

ejecta forms torus shape. These molecules fill in clumps. Three-dimensional models of SN explosions predict that Rayleigh-Taylor instabilities, triggered by the explosion, break the ejecta material into clumps (Wongwathanarat et al. 2015). After over 20 years of evolution in the post-explosion era, high resolution of the ALMA enabled us to resolve these clumps.

\subsection{Cassiopeia A-Forward Shocks, Reverse Shocks and Unshocked Dust}

Cassiopeia (Cas) A is the remnant of Type IIb SN, with an approximate age of 335 years (Fesen et al. 2006; Krause et al. 2008). IRAS (Infrared Astronomical Satellite), an all sky survey mission launched in 1983, detected dust in the SNR, Cas A (Dwek et al. 1987). Together with SN 1987A, detection of dust in this young SNR was one of the milestone discoveries which confirmed that dust can be formed in core collapse SNe.

\subsubsection{SN Dust or ISM Dust?}

The total dust mass in Cas A is largely debated. This is because there is heavy extinction of ISM dust towards Cas A, and evaluation of foreground and background ISM dust could affect Cas A dust mass estimates. The reported dust mass from IRAS was $7 \times 10^{-3} \mathrm{M}_{\odot}$ (Dwek et al. 1987). Using SCUBA/JCMT, a dust mass of 0.2-4 $\mathrm{M}_{\odot}$ was reported in submillimeter wavelength (Dunne et al. 2003). Fitting of the $8-850 \mu \mathrm{m}$ observations including SPITZER MIPS, indicates a presence of $3 \times 10^{-3} \mathrm{M}_{\odot}$ of dust (Hines et al. 2004). AKARI and BLAST $65-500 \mu \mathrm{m}$ observations deduced a 0.003 and $10 \mathrm{M}_{\odot}$ of hot $(99 \mathrm{~K})$ and cold $(17 \mathrm{~K})$ dust (Sibthorpe et al. 2010). The analysis of HERSCHEL observations resulted in $0.075 \mathrm{M}_{\odot}$ of dust with a temperature of $\sim 35 \mathrm{~K}$ (Barlow et al. 2010). Developing SN template analysis on SPITZER and HerSCHEL found $0.04 \mathrm{M}_{\odot}$ of warm SN dust and $<0.1 \mathrm{M}_{\odot}$ of cold dust, whose origin was unclear. It can either be SN ejecta or the ISM (Arendt et al. 2014). After subtracting ISM dust templates from the SED of SPITZER and HERSCHEL, 0.4-0.7 $\mathrm{M}_{\odot}$ of cold SN dust was still present in Cas A in the unshocked region (De Looze et al. 2017).

\subsubsection{Dust Compositions}

Another important finding in Cas A was the spatially varying dust compositions distributed within the SNR and the type of dust compositions correlated to specific atomic lines (Lagage 


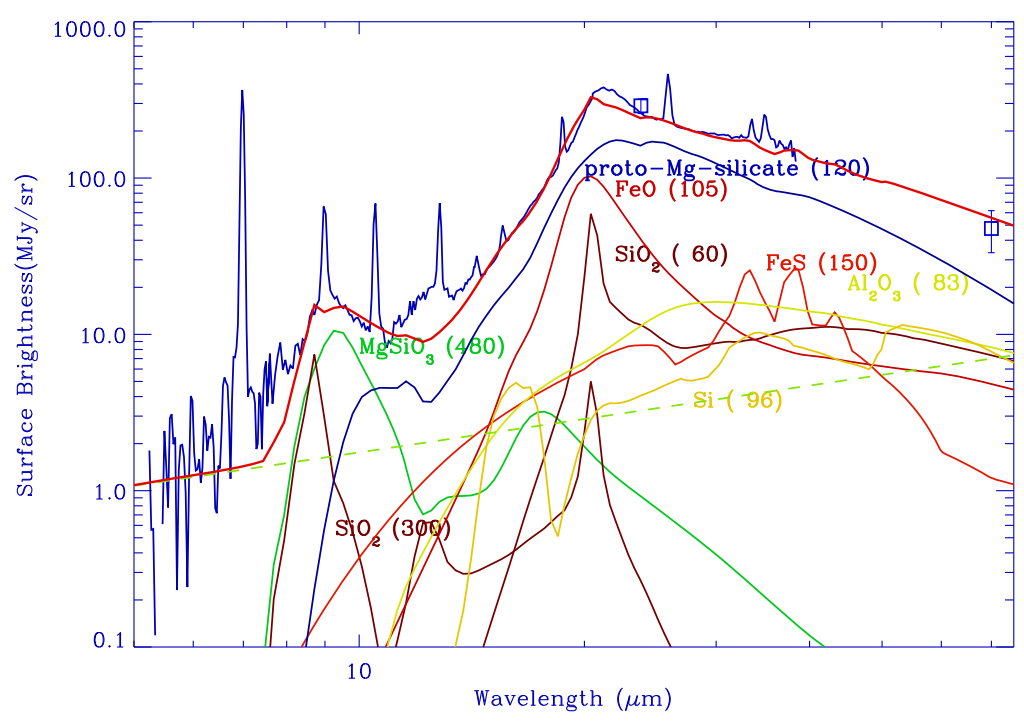

Fig. 7 One of the SPITZER spectra obtained in Cas A. Strong $21 \mu \mathrm{m}$ feature was detected, which was attributed to $\mathrm{Mg}$ protosilicate, amorphous $\mathrm{SiO}_{2}$ and $\mathrm{FeO}$ (Rho et al. 2008)

et al. 1996; Rho et al. 2008; Arendt et al. 2014). The spatially resolved spectra of Cas A showed that the spectra with the ' $21 \mu \mathrm{m}$ feature' are accompanied with a strong [Ar II] line, while the spectra with featureless dust emission is correlated to the [Si II] line (Fig. 7; Arendt et al. 2014; Rho et al. 2008). The ' $21 \mu \mathrm{m}$ feature' could be attributed to a combination of $\mathrm{Mg}$ protosilicate (Arendt et al. 1999), amorphous $\mathrm{SiO}_{2}$ and $\mathrm{FeO}$ (Rho et al. 2008). This is an unique case where a specific dust feature has been identified in SNRs, with recent finding in another Galactic SNR, G54.1+0.3 (Temim et al. 2017).

\subsubsection{CO Molecules in Cas A-Survived Reverse Shocks or Reformed in Shocked Region}

$\mathrm{CO}$ molecules have also been detected in Cas A. Filter measurements at 2 and $4 \mu \mathrm{m}$ suggested the presence of CO emission at the reverse shocked region of Cas A (Rho et al. 2009) and was later spectroscopically confirmed by Rho et al. (2012). One possibility is that the $\mathrm{CO}$ formed in early days might have survived reverse shocks. Alternative possibility is that $\mathrm{CO}$ is re-formed. Wallström et al. (2013) detected high $J$ lines in Cas A. The wide range of temperatures (400-200 K) and high column density $\left(5 \times 10^{17} \mathrm{~cm}^{-2}\right)$ suggests that these CO molecules are from the dense regions of the post-shocked gas, where $\mathrm{CO}$ molecules were reformed.

Chemical models suggest that molecules can re-form in the post-shock region, but not dust (Biscaro and Cherchneff 2014).

\subsection{Crab Nebula}

\subsubsection{Dust in the Crab Nebula}

The SN explosion of the Crab Nebula was recorded in 1054. Unlike Cas A, whose bright optical and X-ray nebula is collisionally heated by reverse shocks (Gotthelf et al. 2001), the 
major heating source of the Crab Nebula is synchrotron radiation (Hester 2008). Dust found in the Crab Nebula was also heated by the synchrotron radiation (Owen and Barlow 2015).

The Crab Nebula is composed of many filament like structures. The wind from the pulsar compresses the expanding ejecta to form these dense filaments. Not only gas but also dust is associated with these filaments (Fig. 8: Fesen and Blair 1990; Gomez et al. 2012; Grenman et al. 2017).

There has been a range of dust masses, that is inferred from observation studies of the Crab Nebula. Marsden et al. (1984) reported thermal emission of dust from IRAS flux measurements at 25, 60 and $100 \mu \mathrm{m}$. The dust mass was inferred to be between $5 \times 10^{-3}$ $0.03 \mathrm{M}_{\odot}$. Green et al. (2004) analysed the Infrared SpaCe ObSERVATORY (ISO)'s 60, 100 and $170 \mu \mathrm{m}$ images, together with the $850 \mu \mathrm{m}$ synchrotron image from James Clerk Maxwell Telescope (JCMT) and estimated a dust mass of $3 \times 10^{-3}-0.07 \mathrm{M}_{\odot}$ with temperatures of 45-50 K. Temim et al. (2006) used SPITZER 24 and $70 \mu \mathrm{m}$ flux, together with ISO's photometric data, resulting in the estimated dust mass of $4 \times 10^{-3}-1.5 \times 10^{-2} \mathrm{M}_{\odot}$ with a temperature of $74 \mathrm{~K}$. Adding the photometric measurements at longer wavelength of 70 $500 \mu \mathrm{m}$ from Herschel SPACE ObSERVATORY, Gomez et al. (2012) found that the dust emission from the Crab Nebula is much broader than a single temperature black body/grey body, which previous authors have used. Using a two component black body, Gomez et al. (2012) found that while their hot component has similar dust temperature and mass as those derived by Temim et al. (2006). Gomez et al. (2012) found that the additional cold component, emitting at longer wavelengths, is at a mass of $0.11-0.24 \mathrm{M}_{\odot}$ with temperatures of 28-34 K. However, the question is if mid-infrared emission from warm dust can hide emission from cold dust, which dominates the total dust mass.

In order to fit the broad emission, Temim and Dwek (2013) invoked temperature gradient of dust grains because synchrotron radiations can make small grains hotter than larger grains due to difference in heat capacities and absorption coefficients. The resultant dust mass is comparable to the estimates from two black bodies (Gomez et al. 2012), as far as the same dust emissivities are chosen. Temim and Dwek (2013)'s model assumed that all dust grains are located at a fixed distance. Owen and Barlow (2015) advanced the distance issue, by applying full radiative transfer modelling to shell structure. Their fitted results on both ionised lines and dust emissions show that the dust mass ranges between $0.17-1.5 \mathrm{M}_{\odot}$ depending on the morphologies of the dust species and their emissivities.

\subsubsection{Molecules in the Filaments of the Crab Nebula}

Vibrationally excited molecular hydrogen lines have been detected in the Crab Nebula (Graham et al. 1990), and the $\mathrm{H}_{2}$ emission originates in the filaments (Loh et al. 2011). Loh et al. (2012) estimated the excitation temperature of $\mathrm{H}_{2}$ to be $2000-3000 \mathrm{~K}$, which is most likely collisionally excited.

The first detection of noble molecular gas in space was made in the Crab Nebula, by the detection of 618 and $1235 \mathrm{GHz}$ lines of ${ }^{36} \mathrm{ArH}^{+}$found in the HERSCHEL Space Observatory's spectra (Barlow et al. 2013). This is an unusual case because majority of molecules had been found first in interstellar medium (ISM) or circumstellar medium of evolved stars and later identified in SNRs. On the other hand, this molecule was reported in a SNR first and then identified later in the ISM (Schilke et al. 2014). Argon is a noble gas, i.e. nonreactive, but its ionised state $\mathrm{Ar}^{+}$is extremely reactive and its reaction with $\mathrm{H}_{2}$ leads to the formation of $\mathrm{ArH}^{+}$. 


\subsection{Galactic and Magellanic SNRs}

Infrared surveys enabled to detect and analyse Galactic and Magellanic clouds SNRs with possibilities to analyse the dust within. Launched in 1983, the IRAS is the first whole sky survey at 12, 25, 60 and $100 \mu \mathrm{m}$ and detections of 51 and 11 SNRs were reported from the survey (Arendt 1989; Saken et al. 1992). These achieved systematic studies of infrared emission from SNRs.

Launched in 2003, the SPITZER SPACE TElesCOPE used Galactic plane surveys $\left(|b|<1^{\circ}\right.$ and $10^{\circ}<|l|<65^{\circ}$; e.g. Benjamin et al. 2003) with two different instruments, IRAC and MIPS. These survey data were used to detect infrared emission from SNRs. Compared to the pixel scale of 2' of the IRAS map used by Arendt (1989), 2.5 arcsec resolution at 3.5-8.0 $\mu \mathrm{m}, 6$ arcsec at $24 \mu \mathrm{m}$ and 20 arcsec at $70 \mu \mathrm{m}$ are great improvements, which helped in resolving ISM cirrus confusion towards the SNRs. Reach et al. (2006) found 17 detections at 3.5-8.0 $\mu \mathrm{m}$ and colour analysis suggested that four of the SNRs appeared to have infrared emission associated with polycyclic aromatic hydrocarbons (PAHs). The emission of the remaining thirteen $\mathrm{SNe}$ were found to be due to molecular lines $\left(\mathrm{H}_{2}\right.$ and $\mathrm{CO}$ ) or atomic lines ([Fe II], [Ar II] and $\mathrm{Br} \alpha$ ) caused by shocks. Pinheiro Gonçalves et al. (2011) searched for counterparts of Green's SNR catalogue (Green 2004) and reported the detection of 39 SNRs at 24 and $70 \mu \mathrm{m}$. The mid-infrared emissions of these SNRs have various origins that include dust and lines.

SPITZER's sensitivities and angular resolutions allowed us to study SNRs beyond the Galaxy such as those in the Magellanic Clouds. That includes dedicated studies of individual SNRs, 1E 0102.2-7219 (Stanimirović et al. 2005; Sandstrom et al. 2009) and the studies (Seok et al. 2013; Lakicevic et al. 2015) using the Magellanic Clouds survey (Meixner et al. 2006). Seok et al. (2008) also studied Magellanic Clouds SNRs using the AKARI infrared whole sky survey. Correlation between infrared emission and X-ray emission suggests that 24 and $70 \mu \mathrm{m}$ flux of the SNRs are collisionally heated (Seok et al. 2015) with the possibility of dust destruction by sputtering (Williams et al. 2006).

\section{Extra-Galactic SNe}

The detections of ejecta dust in SNe beyond the magellanic clouds have been reported by finding asymmetric structures in the optical emission lines (e.g. Elmhamdi et al. 2003b). After the explosion, the ejecta is characterised by broad emission lines and at some stage the blue wing of the broad ejecta lines might be affected by extinction. This extinction indicates the formation of dust in the ejecta. Additionally, sharp decrease in the optical light curve can be found (Elmhamdi et al. 2004). These two methods have been effective to detect ejecta dust in extragalactic SNe.

Presence of high sensitivity instruments in Spitzer Space Telescope and the development of mid-infrared instruments on eight meter telescopes enabled to detect mid-infrared thermal emission of dust from extragalactic SNe (e.g. Sugerman et al. 2006). Detections of midinfrared dust emission help to quantify the dust masses and temperatures. Typical ejecta dust masses reported in SNe younger than day 10,000 are between $10^{-6}-10^{-3} \mathrm{M}_{\odot}$ (e.g. Sugerman et al. 2006; Meikle et al. 2007; Kotak et al. 2006; Fabbri et al. 2011; Szalai et al. 2016) and it has been suggested that the dust masses increase in time (Gall et al. 2011, 2014). Because the measurements of late phase dust masses are limited to a few SNe (e.g. SN 1987A and 1995N; Matsuura et al. 2015; van Dyk 2013) with a large gap in epochs, it 
Fig. 8 The Hubble Space TELESCOPE optical image (blue-white) with Herschel Space Observatory image (red) of the Crab Nebula. Copy right: Oli Usher (UCL); Herschel Space Observatory, Hubble Space Telescope: ESA, NASA. Published in Owen and Barlow (2015). Publication of HST image?

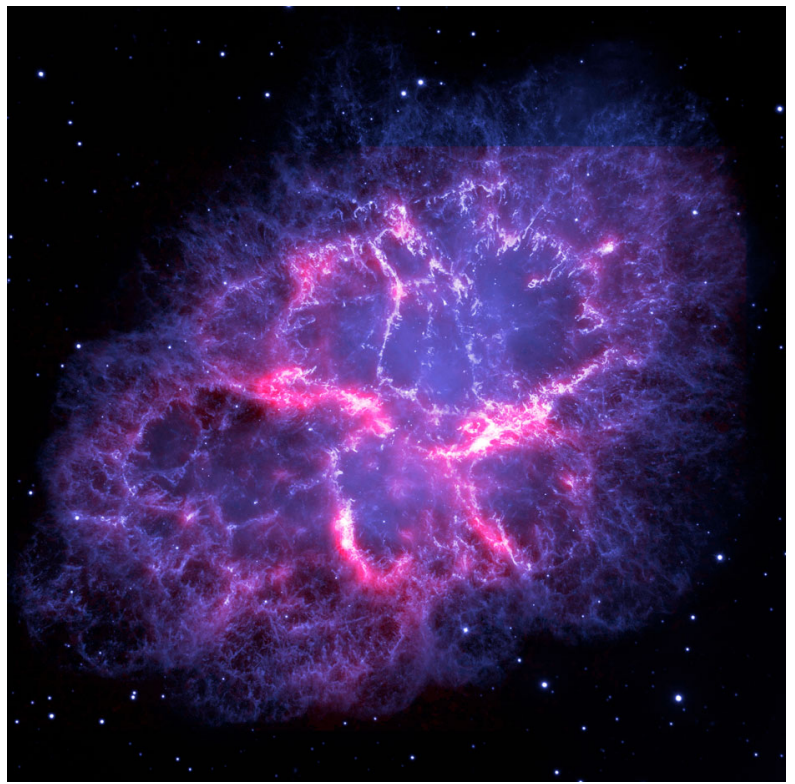

is not yet clear whether the dust masses in the early phase is actually such a small quantity or a large mass of dust was missed due to being optically thick (Sect. 2.1.3).

The dust thermal emission can be associated with ejecta dust, light echo, circumstellar dust or dust from circumstellar material. Often the timing of the appearance together with optical line extinction or optical light curves are used in order to identify the origin of dust emission.

\subsection{Molecules as Precursor to Dust Formation?}

By exploiting extra-galactic $\mathrm{SNe}$, the number of $\mathrm{SNe}$ where molecules and dust have been detected has increased. Chemical models predict, the time sequence of formation of molecules in $\mathrm{SNe}$ is followed by dust formation. The binding energy of $\mathrm{CO}$ is one of the highest among molecules found in astronomical environment, and formation of $\mathrm{CO}$ starts about day $\sim 200$ with a sharp increase in mass up to day 500, followed by a gradual increase up to day 800 (Sect. 6.2.2: Sarangi and Cherchneff 2013). After that, the CO mass does not increase so much. Whereas the dust condensation starts at about day 200 , followed by silica formation at day 400 . We test this scenario by looking into the past observations of molecules, mainly $\mathrm{CO}$, and dust.

First, we test link between molecule and dust formation. So far, molecular detections were reported in the spectra of twelve SNe (Summarised in Table 1; extracted from Matsuura 2017). CO detections suggested in photometry measurement only are excluded from the analysis. SN 1987A is included among the twelve, but not SNRs such as Cas A and the Crab Nebula. Figure 9 shows the statistics of dust detections out of twelve SNe. We divide the sample into two parts-six SNe before the Spitzer mission and six SNe after the Spitzer mission. The latter includes SN 2002hh, whose explosion was recorded before Spitzer launch in 2003, but has been observed with Spitzer, and SN 2013eg, which was type Ic and detected after Spitzer cryogenic phase. 


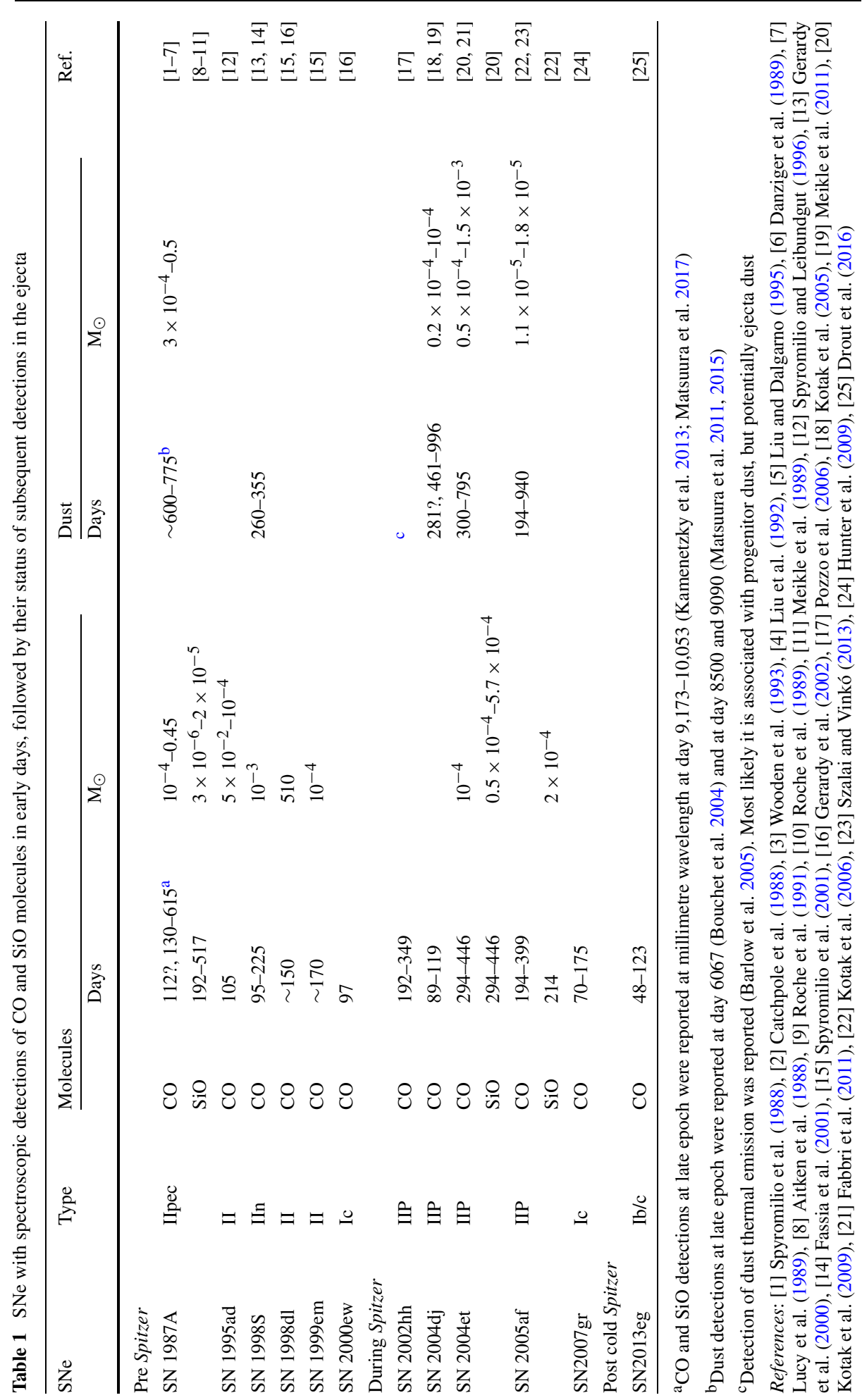


Fig. 9 Statistics of CO detected $\mathrm{SNe}$, highlighting whether they were followed by dust detections or not

Fig. 10 Histograms indicating the reported days of molecular and dust detections in $\mathrm{SNe}$
Pre Spitzer (before 2003)

Post Spitzer (after 2003)

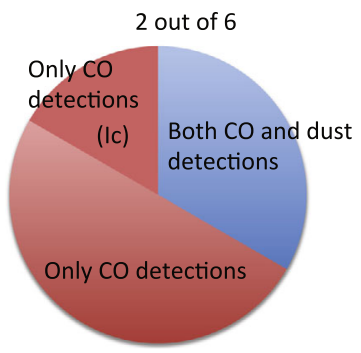

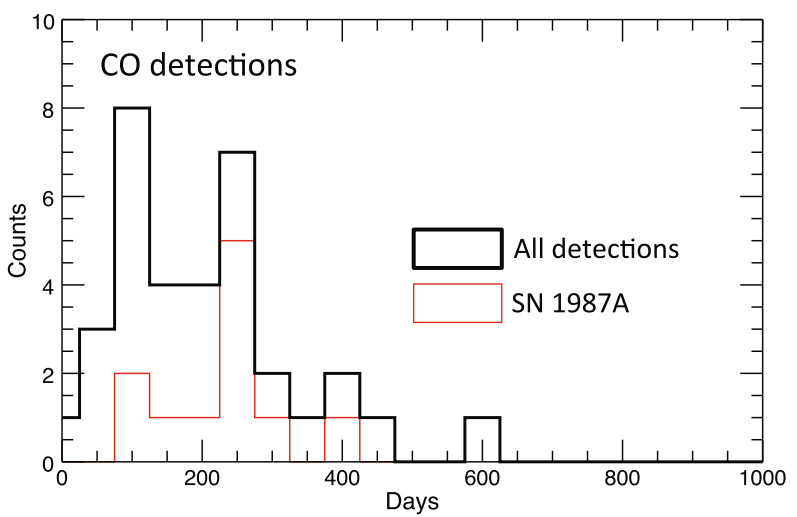

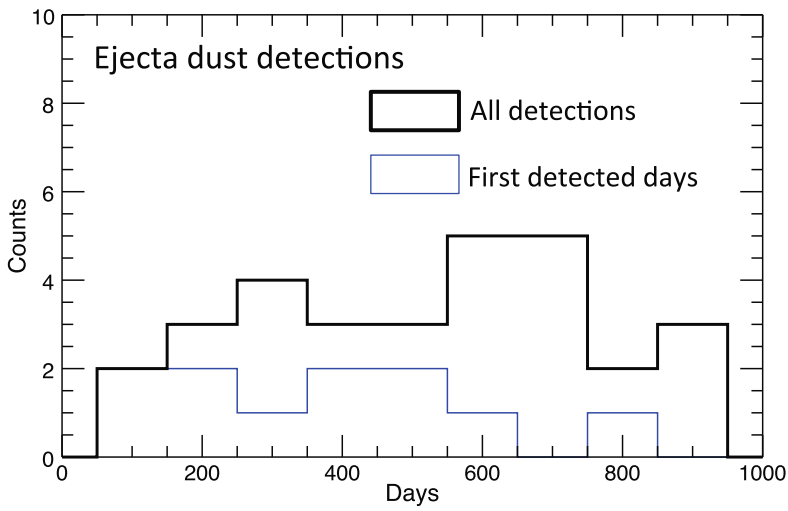

Out of the twelve SNe with molecular detections, dust detection was reported in six $\mathrm{SNe}$ (Fig. 9). Three of the no-dust detected SNe are of Type Ic and Ib/c SNe, which have fewer dust detections reported so far (Matsuura 2017). Other SNe are all Type II, mainly Type IIP, with one IIn and unclassified subclass (Table 1). From Type II, dust detection was reported in six SNe out of nine, making the succession of molecule formation followed by dust formation very likely. In particular, after the launch of Spitzer, all Type II SNe with molecular detections ended with dust detections, so this scenario seems very likely.

Next, we test the timing of molecule and dust formation/detections. We assumed the reported days of $\mathrm{CO}$ detections and ejecta dust detections from Matsuura (2017) and the histogram of these days are plotted in Fig. 10. CO detections from one SN at different 
epochs are counted individually. In these panels, $\mathrm{CO}$ and ejecta dust detections in late epoch (>1000 days) of SN 1987A are not plotted.

The histogram (Fig. 10) shows that $\mathrm{CO}$ detections have been reported about 100-300 days. These are mainly detections at $2.3 \mu \mathrm{m}$ and $4.6 \mu \mathrm{m} \mathrm{CO}$ fundamental and the firstovertone band. After that period, $\mathrm{CO}$ emission fades, making it difficult to detect at nearinfrared wavelengths. The second highest peak at 225-275 days is due to five separate $\mathrm{CO}$ observations of SN 1987A, distorting the frequency of CO detection rate during this period. While the ejecta gas cools down, the gas can not excite vibrational level of $\mathrm{CO}$, so that nearinfrared vibrational CO bands disappear by $\sim 600$ days. Instead, CO rotational lines appear at millimetre wavelength ranges and is at least reported by day $\sim 9000$ (Kamenetzky et al. 2013; Matsuura et al. 2017).

While CO detections have a gentle peak between 100-300 days, any such peak is not found in the timings of the ejecta dust detections. In Fig. 10, records of first ejecta dust detection per SN are plotted in blue and these reported first detections spread across 100800 days. Dust can be associated with ejecta, light echo, circumstellar and ISM dust, and it is not always easy to disentangle these cases. Nevertheless, typically the highest chance of the ejecta dust detection appears to be between 600-700 days.

\section{Indirect Evidence of Supernova Dust}

Apart from direct observations of dust signatures in the mid-IR and submillimeter wavelengths in different local SNe as discussed above, there are also some indirect yet strong evidences of dust in $\mathrm{SNe}$, which helps us understand and constrain the mechanism of dust formation in such environments. Among them, the dust case of high redshift galaxies (Maiolino et al. 2004; Dwek and Cherchneff 2011) and the isotopic anomalies in presolar grains from meteorites (Clayton and Nittler 2004; Ott 2001) provide us with a lot of important information on $\mathrm{SNe}$ dust, as explained below.

\subsection{The High Red-Shift Galaxies}

The reddening of the background quasars and Ly $\alpha$ systems at high redshift indicates the presence of dust in those galaxies (Pei et al. 1991; Pettini et al. 1994; Hines et al. 2006). Large masses of dust of the order $1-5 \times 10^{8} \mathrm{M}_{\odot}$ is estimated to be present in Sloan Digital Sky Survey (SDSS) J1148+5251, a hyperluminous quasar at $z=6.4$ (Bertoldi et al. 2003; Dwek et al. 2007). Figure 11 points to the high redshift galaxies on the cosmic time scale where presence of large dust masses is reported. The presence of large masses of dust plays pivotal role in the cooling of the primeval large gas clouds, eventually leading to the formation of next generation of stars (Cherchneff 2013). The Population III stars present in these galaxies are predominantly massive and a large section is assumed to be in binary form. The sources of such large masses of dust and the formation mechanisms are yet to be understood with confidence. Generally supernovae and AGB stars are taken as the prime dust producers in the galaxy. In order to explain the large masses of dust at high redshift galaxies, Dwek and Cherchneff (2011) assumed the contribution from all possible dust producers in the galaxy and its balance with the galactic star formation rate. Massive stars evolve much faster compared to the low mass stars. Therefore, in the timescales of the lifetime of the early galaxies, supernovae are estimated to be the main source of dust. If $1 \mathrm{M}_{\odot}$ of dust is produced by corecollapse supernovae before getting reprocessed in the remnant phase and a top-heavy initial 


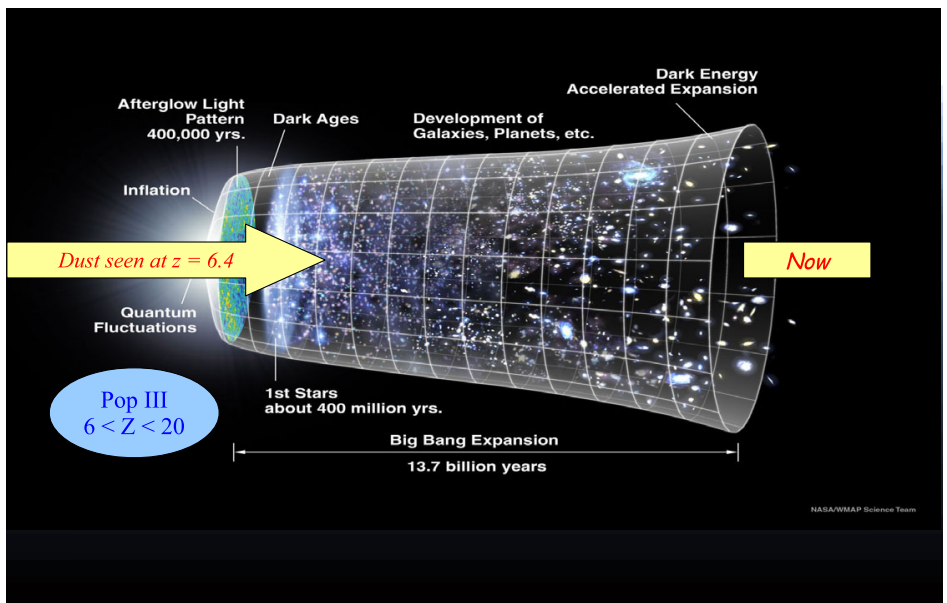

Fig. 11 Evidence of large masses of dust reported from the far-IR spectrum of hyperluminous galaxy SDSS $\mathrm{J} 1148+5251$ at high redshift $z=6.4$ (Bertoldi et al. 2003; Dwek et al. 2007) (Photo courtesy: NASA WMAP)

mass function is assumed for the primeval stars, then the dust masses observed at high redshift galaxies can be explained. However, theoretical models infer a mass of $0.1-0.15 \mathrm{M}_{\odot}$ of silicate dust formed per massive pair-instability supernova as an upper limit (Cherchneff and Dwek 2009; Cherchneff 2010). Moreover, the destruction of dust by the reverse shock in the ejecta or by UV photolysis in the ISM are somewhat underestimated. AGB stars can only contribute as a major dust producer if the galactic age is assumed to be much larger (400 Myrs). Therefore, in case of high z galaxies, SNe are expected to have obvious advantages (Maiolino et al. 2004; Calura et al. 2014; Gall et al. 2011) over AGB stars in terms of relative contributions owing to the large abundances of massive stars and their shorter evolutionary timescales (Dwek 1998; Dwek and Cherchneff 2011).

\subsection{Presolar Stardust from Meteorites}

Laboratory studies have revealed the presence of surviving dust grains of circumstellar origin within primitive meteorites (Bernatowicz and Zinner 1997). The deviation from solar isotopic composition confirms the presolar origin of these dust particles. Further, depending on the isotopic abundances, the stellar source of the grains as well as some limitations on the physical conditions of the environment can also be inferred (Hoppe and Ott 1997). Dust formed in the ejecta of a supernova carries signatures of the nuclear processes from the pre-explosion and post-explosion phases of the progenitor (Ott 2001).

Out of several types of chemical species found in the meteorites, silicon carbide grains have been profoundly studied and grouped into categories (A, B, Y, Z, X etc.) depending on their possible sources of origin (Hoppe and Ott 1997). The presolar SiC grains of type X ( $\sim 1 \%$ of all presolar SiC samples collected from meteorites) has been attributed to have $\mathrm{SN}$ origin (Amari et al. 1997), as shown in Fig. 12, based on the enrichment of ${ }^{12} \mathrm{C}$ and ${ }^{15} \mathrm{~N}$, high abundance of ${ }^{28} \mathrm{Si}$, and importantly the presence of ${ }^{44} \mathrm{Ca}$, a remnant of short-lived radioisotope ${ }^{44} \mathrm{Ti}$ (Ott 2001; Hoppe 2010). Similar to silicon carbide, a few of the silicon nitride $\left(\mathrm{Si}_{3} \mathrm{~N}_{4}\right)$ samples found also have isotopic signatures of that of $\mathrm{SNe}$ (Amari et al. 1997). Apart from these, a small subsection of presolar aluminum oxide grains (Nittler et al. 1998; 
Fig. 12 The categorisation of presolar $\mathrm{SiC}$ grains based on ${ }^{14} \mathrm{~N} /{ }^{15} \mathrm{~N}$ and ${ }^{12} \mathrm{C} /{ }^{13} \mathrm{C}$ ratios (Figure adopted from: Zinner et al. 2010, Hynes and Gyngard 2009)

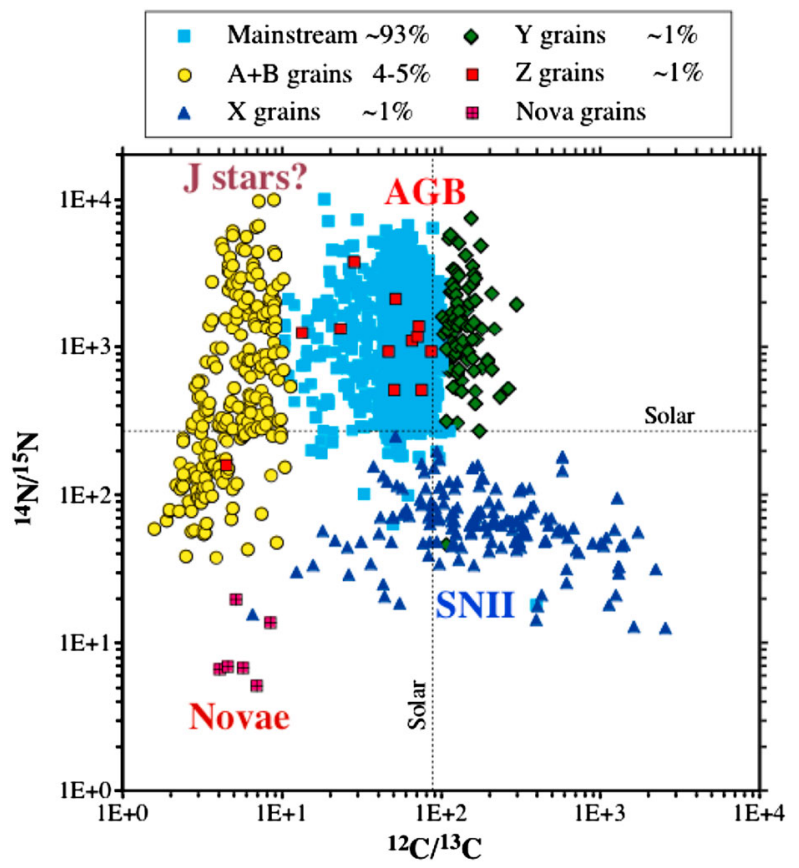

Choi et al. 1998) and some of the low-density graphite grains from Murchison carbonaceous meteorite (Travaglio et al. 1999) are estimated to be of SN origin. Recent detection of ${ }^{18} \mathrm{O} /{ }^{16} \mathrm{O}$ enhancement in a few of the silicate samples from meteorites additionally claims the presence of Mg-O-silicates in SNe (Leitner et al. 2015).

Even though, SN has been established as a source of cosmic dust in many previous analyses, the isotopic compositions of the grains are not exactly coherent with the existing theoretical models (Herant and Woosley 1994) and also observational studies of the SN ejecta (Hughes et al. 2000). In order to match the isotopic signatures, selective yet microscopic mixing between the ejected materials from different zones of the ejecta is necessary (Nittler 2003; Zinner et al. 2010). In order to understand the mixing scales compatible with the isotopic compositions in the grains, observations of the $\mathrm{SN}$ ejecta at high spatial resolutions are required. New theoretical studies of the post-explosion ejecta using 3D hydrodynamic models (Hammer et al. 2010b; Wongwathanarat et al. 2015) also hint at more intense mixing between the stratified zones.

\section{The Chemical Nature of Dust Grains}

Compared to dust in earth's atmosphere, dust grains in space have relatively simpler chemical forms as they exist in more hostile and pristine surroundings. The chemical types of cosmic dust and their precursor molecules are not well defined through observations due to lack of high-resolution mid-IR data (Cherchneff 2013). Composition of dust in space can be analysed by (a) the laboratory study of presolar grains from meteorites, (b) fitting of mid-IR spectral energy distribution using known opacities of probable dust species, (c) modelling of dust condensation from its gas phase precursors. Cosmic dust from circumstellar sources can be classified chemically as (a) oxides (silicates of quartz or metal oxides), (b) carbides, 
Table 2 Circumstellar dust types derived from mid-IR $(5-40 \mu \mathrm{m})$ spectra through laboratory experiments (Adopted from Cherchneff 2013)

\begin{tabular}{|c|c|c|c|c|}
\hline Family & Name & Formula & Bands $(\mu \mathrm{m})$ & Reference \\
\hline \multicolumn{5}{|l|}{ Oxides } \\
\hline \multirow[t]{6}{*}{ Silicates } & Pyroxene & $\mathrm{Mg}_{x} \mathrm{Fe}_{1-x} \mathrm{SiO}_{3}$ & $10 ; 20$ & Dorschner et al. (1995) \\
\hline & Enstatite & $\mathrm{MgSiO}_{3}$ & $9-12 ; 15.4 ; 19.5 ; 36.2$ & Chihara et al. (2002) \\
\hline & Ferrosilite & $\mathrm{FeSiO}_{3}$ & $11.3 ; 20.4 ; 31.7$ & Chihara et al. (2002) \\
\hline & Olivine & $\mathrm{Mg}_{2} x \mathrm{Fe}_{2-2 x} \mathrm{SiO}_{4}$ & $10 ; 20$ & Dorschner et al. (1995) \\
\hline & Fosterite & $\mathrm{Mg}_{2} \mathrm{SiO}_{4}$ & $10-12 ; 16.3 ; 19.5-24$ & Koike et al. (2003) \\
\hline & Fayalite & $\mathrm{Fe}_{2} \mathrm{SiO}_{4}$ & $10 ; 18-22 ; 27 ; 32$ & Suto et al. (2002) \\
\hline Quartz & Silica & $\mathrm{SiO}_{2}$ & $9.1 ; 12.6 ; 20.4-21.2 ; 26.1$ & Fabian et al. (2000) \\
\hline \multirow[t]{7}{*}{ Metal oxides } & Alumina & $\mathrm{Al}_{2} \mathrm{O}_{3}$ & 13 & Koike et al. (1995) \\
\hline & Spinel & $\mathrm{MgAl}_{2} \mathrm{O}_{4}$ & $13 ; 16.8 ; 32$ & Fabian et al. (2001) \\
\hline & Magnesia & $\mathrm{MgO}$ & 19 & Henning et al. (1995) \\
\hline & Wüstite & $\mathrm{FeO}$ & 23.4 & Henning et al. (1995) \\
\hline & Hematite & $\mathrm{Fe}_{2} \mathrm{O}_{3}$ & $9.2 ; 18 ; 21 ; 20$ & Koike et al. (1981) \\
\hline & Magnetite & $\mathrm{Fe}_{3} \mathrm{O}_{4}$ & $17 ; 25$ & Koike et al. (1981) \\
\hline & Calcium oxide & $\mathrm{CaO}$ & 31.4 & Hofmeister et al. (2003) \\
\hline
\end{tabular}

Carbon

$\begin{array}{llll}\text { Amorphous carbon } & \mathrm{C} & 6.2 ; 8 & \text { Colangeli et al. (1995) } \\ \text { Graphite } & \mathrm{C} & 6.3 ; 11.52 & \text { Draine (1984) }\end{array}$

Carbides

$\begin{array}{llll}\text { Silicon carbide } & \mathrm{SiC} & 11.3 & \text { Mutschke et al. (1999) }\end{array}$

Sulphides

$\begin{array}{llll}\text { Magnesium sulphide } & \mathrm{MgS} & 25-39 & \text { Hofmeister et al. (2003) } \\ \text { Iron sulphide } & \mathrm{FeS} & 23 ; 34 ; 39 & \text { Hofmeister et al. (2003) }\end{array}$

(c) sulphides, (d) homogeneous elemental clusters. Further they can be either crystalline or amorphous in nature depending on the physical conditions of the environment at the time of their synthesis. Stretching and bending modes of vibration within the grains of a given type give rise to their unique emission or absorption features. The dust species can be identified by their mid-IR spectroscopic signatures through laboratory measurements, which are heavily dependent on the proper estimation of the optical properties of the grains. The HJPDOC database (developed by the Astrophysical Institute of the Friedrich Schiller University, Jena, Germany and the Astronomical Institute of the St. Petersburg University, Russia) provides information about a large set of optical constants necessary to analyse cosmic dust and its properties. Table 2, adopted from Cherchneff (2013), gives a summary of known dust species and their mid-IR bands with the references.

In case of SN ejecta, hydrogen mixing is understood to occur in macroscopic scales only (Joggerst et al. 2010; McCray 1993). Therefore, dust formation pathways are controlled by simplified $\mathrm{H}$-free chemistry leading to a small set of possible dust species. From bottomup perspectives, the ejecta chemistry is modelled to predict the types and abundances of molecules and dust grains in the metal-rich ejecta (Bianchi and Schneider 2007; Cherchneff and Dwek 2009; Sarangi and Cherchneff 2013; Sluder et al. 2016). However, in order to conclusively comment on the type(s) of dust present in a certain environment, radiative transfer 
(RT) modelling of the ejecta and fitting of the observed spectra are required (Sugerman et al. 2006; Ercolano et al. 2007). For optically thin media, the spectral features of the dust grains are prominent whereas in case of large optical depths, the dust continuum behaves more like a black body spectrum making the detection of a particular dust type more ambiguous. In the following sections of this chapter, the theoretical aspects of the study of SNe have been addressed in more detail.

\section{Theoretical Models}

Observational studies have confirmed the presence of dust signatures in SN ejecta, which can either be contribution of echo from pre-existing dust or newly formed dust in the ejecta. In order to understand the origin of dust in such environments, we shall now discuss the mechanisms which aid the synthesis of dust grains in SN ejecta.

Attempts to theoretically model an astrophysical environment or event focus on the temporal and spatial variance of the following: (a) the morphology/geometry, (b) relevant physical processes, (c) chemical pathways. To study these aspects with respect to a typical SN ejecta, several simplifications/ approximations are necessarily imposed on the models. The following subsections describe the existing models of SN ejecta and their pertinence compared to the actual environments.

\subsection{Models Based on CNT and LTE}

The earliest approach to model dust synthesis in SN ejecta involved a Classical Nucleation Theory (CNT) based formalism. The CNT based approach was developed using the 'liquiddrop model' introduced to study water droplets in earth's atmosphere (Feder et al. 1966). In this model, the nucleation of gas-phase metals is addressed by a free-energy controlled nucleation current that leads to the formation of critical clusters. The initial growth of these clusters by the attachment of new molecules is an energetically unfavorable process as the surface energy of these species (the missing binding energy of surface molecules) exceeds the volume binding energy. The work required to form a single cluster of k-monomers is a function of (a) the change in the energy of molecules due to its transition from gas to solid phase, (b) the binding energy of the involved species, and (c) the surface energy of the cluster. In macroscopic scale, the energy to form a cluster of k-monomers is equal to the change in the Gibbs free energy of a gas containing k-number of monomers that is undergoing a phase transition at a given pressure and temperature, which is given as,

$$
W(k)=\Delta G_{k}=-k_{B} T \ln \mathcal{S} k+u_{s} k^{2 / 3},
$$

where $W(k)$ is the work required, $k_{B}$ is the Boltzmann constant, $\mathcal{S} \equiv P / P_{v}$ is the supersaturation ratio and $u_{s}$ is the surface energy term.

These supersaturated compounds (the critical clusters), synthesized in this process, are assumed to be in thermodynamic equilibrium (TE) with the solid phase of dust. The accretion and detachment rates of gas monomers are therefore calculated considering a steady state environment.

Dust formation in SNe was discussed in an empirical approach by Clayton (1979). Thereafter, a pioneering attempt to model dust formation was made by Kozasa et al. (1989) based on the early results of SN1987A. This study considered a fully microscopically mixed ejecta where the gas and dust phase was connected by CNT and TE assumptions. Following that, 
CNT based models were applied to study dust formation in SNe (CCSN \& PISN) by Todini and Ferrara (2001), Schneider et al. (2004), Bianchi and Schneider (2007), and more recently by Marassi et al. (2015) for various metallicities and mass of progenitors as well as for various levels of mixing.

Models based on kinetic nucleation theory (KNT) provide an alternative formalism to study dust formation in such environments. In this approach, the number densities of clusters are traced explicitly. The condensation of grains is computed using accretion, where as the destruction of clusters occur through evaporation. The rate coefficients are calculated using kinetic balance. Therefore, it does not necessarily consider a steady state environment for the nucleation processes. Several studies over the last decade have applied this approach to model dust formation in SN ejecta (Nozawa et al. 2003, 2008; Nozawa and Kozasa 2013).

However, the use of nucleation theory to study dust formation in environments such as SN ejecta was questioned first by Donn and Nuth (1985) and later elaborated by Cherchneff and Lilly (2008). CNT is applicable to steady-state conditions, which are usually not found in dynamic environments out of equilibrium (Cherchneff and Dwek 2009). All CNT-based models ignore the non-equilibrium chemistry related to the formation of molecules and dust clusters and the specific physics of ejecta where radioactivity greatly impacts the gas-phase chemistry through cascades of Compton electrons. Moreover, the CNT approach includes the quantities like specific surface energy, sticking coefficient, supersaturation ratio, partial vapour pressure, critical clusters, etc. in analysing the dust formation scenario. These all are essentially bulk properties to matter. However, the critical clusters sizes are of molecular dimensions and therefore, application of the bulk properties like surface energy or sticking coefficient on such small particles is not appropriate (Cherchneff 2013). Additionally, in CNT-models, the condensation sequence is based on assuming condensation temperatures at equilibrium as references. Estimating the condensation temperatures at equilibrium for the seed nuclei is also not an appropriate approach in most cases, as the temperatures of the newly formed grains heavily depend on the ambient gas temperatures and densities, controlled by the amount of radioactive ${ }^{56} \mathrm{Ni}$ produced at the explosion. Even though the KNT based models can take the non-steady state environment into account, they still ignore the actual chemical pathways that lead to the synthesis of the dust-seed nuclei. Further, important condensation mechanisms such as coagulation are also not included in such analyses.

\subsection{Chemical Kinetics Model}

A formalism based on chemical kinetics can overcome most of the limitations imposed by previous models. In this case, dynamics and chemistry control the synthesis of condensates (Cherchneff and Lilly 2008; Cherchneff and Dwek 2009, 2010; Sarangi and Cherchneff 2013, 2015; Biscaro and Cherchneff 2014, 2016; Sluder et al. 2016). A chemical kinetic approach deals with the dynamics of chemical reactions and the conditions affecting the reaction rates. It is controlled by the concentration of the reactants, temperature of the medium and the enthalpy of the individual reactions. The SN ejecta has a very dynamic and unstable physical environment. The passage of the supernovae blast wave through the helium core and the deposition of radioactive energy in the ejecta creates a complex physical environment whose chemistry is neither at steady state nor can be assumed to be at thermodynamic equilibrium (TE). Following the explosion, the ejecta moves at a velocity $\sim 2000 \mathrm{~km} / \mathrm{s}$ and the density and temperature of the ejecta drop rapidly as power-laws. The rich chemical composition of the ejecta is controlled by a large set of chemical reactions with diverse reaction rates. All these chemical reactions occur simultaneously and are coupled to each other. Each individual species is linked to the chemical network through a series of formation and destruction pathways, which comprise of both thermal and non-thermal processes. 


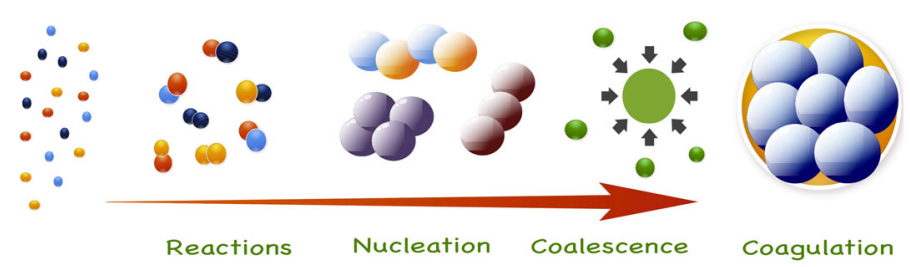

Fig. 13 A cartoon shows the chemical paths leading to the synthesis of molecules, clusters and dust grains in the ejecta through nucleation and condensation phases

Therefore, with the alteration of temperature and density in the ejecta, the relative importance of specific reactions in the network may also change. These factors not only ensure the complexity of the expected chemical yields but also create a case which is far from the steady state condition.

The chemical pathway proceeds through simultaneous phases of nucleation and condensation (Fig. 13). The nucleation phase comprises of chemical reactions which lead to the synthesis of molecules and small clusters. The condensation phase couples the gas phase chemistry to the solid phase of dust. The small clusters formed in the gas phase condense through coagulation and coalescence to form large grains of dust, provided suitable conditions are prevalent. Therefore, we first discuss the physical environment of a typical SN ejecta before going through the detail chemical scheme.

\subsubsection{Physical Conditions in the Ejecta}

We choose a typical $15 \mathrm{M}_{\odot}$ Type IIP SN as a standard case study considering Type IIP SN ejecta to be the most conducive to dust formation. The ejecta of a typical PISN has some morphological differences with a CCSNe owing to the metallicity of the Population III stars. The contrasts in the physical conditions of such objects will be addressed separately. For the sake of simplicity, a 1D stratified model of the ejecta is assumed and we shall ignore the details of the explosion mechanism in this discussion. The abundances of post-explosion elements are provided by nucleosynthesis studies and hydrodynamic simulation models of the ejecta (Woosley and Weaver 1995; Thielemann et al. 1996, 2011; Thielemann 2017; Limongi et al. 2000; Rauscher et al. 2002). The final yields from these models correspond to a timescale of 6-7 hours from the explosion. These elemental yields are then used as initial inputs to the chemical models of the ejecta (Rauscher et al. 2002).

Following the expulsion of the helium core, the ejected materials are crossed by the blast wave, which deposits energy in the gas. The neutrino-driven convection during the collapse leads to the Rayleigh-Taylor instabilities and mixing in the helium core. The mixing scenario continues over a few hours after the explosion and leads to partial fragmentation of the core (Joggerst et al. 2010). Thereafter, the chemical species produced by nucleosynthesis flows ballistically as carried by the blast wave. Hence the abundances of the elements presented as functions of mass zones (Rauscher et al. 2002) in the ejecta (Fig. 14) can be safely assumed to remain constant over the next few days until chemical reactions becomes relevant. The explosion energy, $E_{\text {exp }}\left(\sim 10^{51} \mathrm{ergs}\right)$ is assessed using SN1987A as reference (Woosley 1988), which gives an estimate of the ejecta velocity. Based on the distribution of elements, the ejecta can be stratified into several regions or zones, assuming no further microscopic mixing takes place after a few hours from explosion. The ejecta of the $15 \mathrm{M}_{\odot}$ progenitor model is divided into 8 zones according to the abundances of important elements: $1 \mathrm{~A}(\mathrm{Si} / \mathrm{S})$, 1B(Si/O), 2(O/Si/Mg), 3(O/Ne/Mg), 4A(C/O), 4B(C/O/He), 5(He/C) and 6(He/N) $($ Sarangi 
Fig. 14 The post-explosion elemental abundances inside the $\mathrm{He}$-core as a function of mass zones for a $15 \mathrm{M}_{\odot}$ progenitor star which has exploded as a Type IIP supernova (Rauscher et al. 2002)

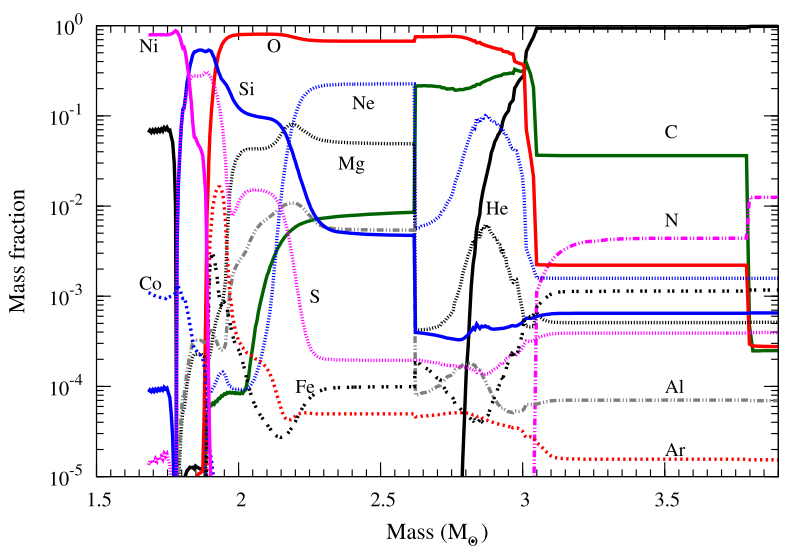

and Cherchneff 2013). In this study, the species in individual zones are assumed to be microscopically mixed. The dominant chemical processes in each zone vary depending on the temperature, density and the abundances.

The SN light curve is powered by radioactive decays (Woosley et al. 1989). The energy deposited by the SN explosion-flash is radiated away in a couple of weeks (Woosley 1988; Blinnikov et al. 2000; Ensman and Burrows 1992; Dwek and Arendt 2008). After that, the bolometric luminosity, which includes the optical, IR and UV emissions, is controlled by interactions between high energy radiations with the supernova ejecta. There are several radioactive nuclei produced in the explosion. On the basis of the abundances and the halflives, the most important radioactive nuclei in the ejecta are ${ }^{56} \mathrm{Ni},{ }^{57} \mathrm{Ni},{ }^{55} \mathrm{Co},{ }^{44} \mathrm{Ti}$ and ${ }^{22} \mathrm{Na}$ (Kozasa et al. 2009). ${ }^{56} \mathrm{Ni}$ has a very short lifetime of 6 days and quickly decays to ${ }^{56} \mathrm{Co}$ through electron capture. ${ }^{56} \mathrm{Co}$ in turn decays to ${ }^{56} \mathrm{Fe}$ with a half-life is 77.12 days. The light curve of the first few years of SN1987A is dominated by ${ }^{56} \mathrm{Co}$ and a mass of $0.075 \mathrm{M}_{\odot}$ reproduces the bolometric luminosity (Woosley et al. 1989). Hence an equal amount of ${ }^{56} \mathrm{Ni}$ is estimated to be produced in the explosion. The ${ }^{56} \mathrm{Co}$ decays through electron capture $\left({ }^{56} \mathrm{Co} \longrightarrow{ }^{56} \mathrm{Fe}+\gamma+v_{e}\right)$ and produces energetic $\gamma$-rays and electron neutrinos. The energetic $\gamma$-rays interact with the ejecta and get degraded to hard X-rays through Compton scattering. The fast Compton electrons thermalize the ejecta through heating, excitation and ionisation (Liu and Dalgarno 1995). The hard X-ray gets further degraded to soft X-rays and UV photons through cascades of inverse Compton scattering and ionization-recombination processes. The entire energy of the $\gamma$-rays is not deposited in the ejecta. Study of the SN1987A light curve indicates the presence of macroscopic mixing of ${ }^{56} \mathrm{Ni}$ in the ejecta, which is also confirmed by the early emergence of $\gamma$-rays and X-rays within a few hundred years from explosion. The ejecta is presumably clumpy and hence a small fraction of the high energy photons can possibly pass through the less dense interclumps unabsorbed (McCray 1993). However, the opacity of the ejecta is found to increase rapidly at early times. Hence, to a first order approximation, the fraction of escaping energy becomes negligible. For the sake of generality, it can be assumed that the entire radioactive energy is uniformly deposited in the ejecta (Cherchneff and Dwek 2009; Sarangi and Cherchneff 2013). The rate of energy deposition $\left(L_{\gamma}\right)$ in the ejecta by ${ }^{56} \mathrm{Co}$ is calculated following the study by Woosley et al. (1989) in terms of the $\gamma$-ray optical depth $\left(\tau_{0}\right), \gamma$-ray mass-absorption coefficient $\left(\kappa_{\gamma}\right)$, the deposition function $(f(\tau))$ and the energy emitted per ${ }^{56}$ Co nucleus $\left(E_{\gamma}\right)$ (Liu and Dalgarno 1994; Cherchneff and Dwek 2009; Sugerman et al. 2006). The rate varies linearly with the 


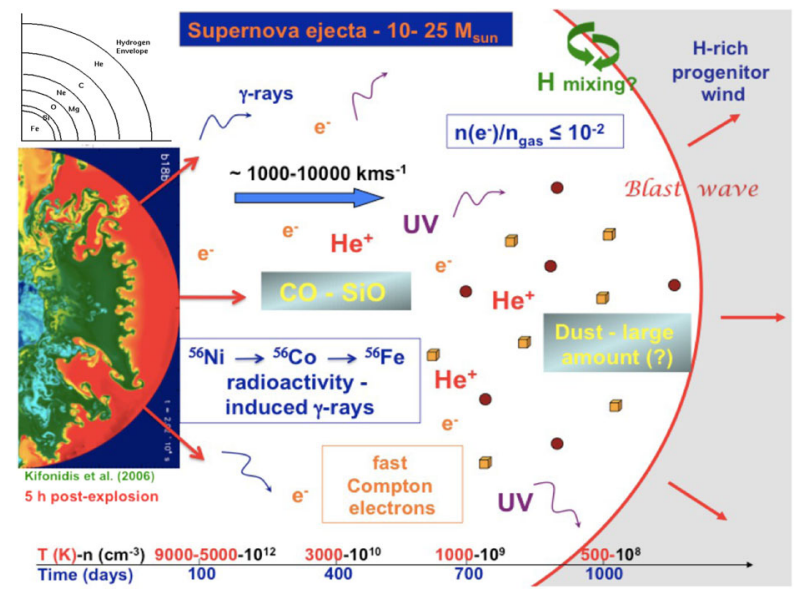

Fig. 15 The schematic diagram, adopted from Cherchneff and Sarangi (2011), represents the time evolution of a typical CCSN ejecta. The scale in the bottom of the figure illustrates the temperature/density variation with post-explosion time. On the top left corner, the stratification of the stellar core and envelope is shown which retains the signatures from the burning phases of the massive star. The mixing and the Rayleigh-Taylor instabilities in the ejecta that occur until a few hours after the explosion, is also presented in the picture, adopted from Kifonidis et al. (2006)

total mass of radioactive ${ }^{56} \mathrm{Ni}$ produced by the explosion (Sarangi and Cherchneff 2013, 2015).

The ejecta is assumed to undergo a quasi-adiabatic expansion (Cherchneff and Dwek 2009) over a span of few years. The temperature profile is obtained with reference to observations and models based on SN1987A (Fransson and Chevalier 1989; Kozasa et al. 1989). The temporal variation of gas temperature in the ejecta is represented by an 'adiabatic' index $\gamma(\sim 1.42)$ for SN Type IIP, as derived by Umeda and Nomoto (2002) \& Nozawa et al. (2010). The propagation of the shock causes a velocity shear in the post-explosion ejecta and within one day of explosion, the ejecta shell expands homologously (Shigeyama and Nomoto 1990; Nozawa et al. 2003). The initial gas densities and temperatures at $\sim$ day 100 (Sarangi and Cherchneff 2013; Biscaro and Cherchneff 2014) used for the model are derived from Nozawa et al. (2010). Figure 15, adopted from Cherchneff and Sarangi (2011), presents a cartoon illustrating the post-explosion ejecta expanding outwards from the stellar remnant.

\subsubsection{Nucleation Phase}

Molecules and clusters act as the building block of dust in supernova ejecta. The nucleation scheme, which deals with the formation and destruction of such species in the gas phase, therefore acts the bottleneck to the entire mechanism (Cherchneff and Dwek 2010; Sarangi and Cherchneff 2013). The He-core, rich in metal concentration and poor in $\mathrm{H}$ abundance, is the prime site of dust synthesis in SN ejecta. The environment in concern being H-poor, simplifies the relevant chemical pathways to a great extent. Moreover it diminishes the variety of chemical compounds synthesized as intermediate or end products. Table 3 provides the list of all possible chemical species $(\sim 120)$ in the gas phase that are important for this study (Cherchneff and Dwek 2009; Sarangi and Cherchneff 2013; Biscaro and Cherchneff 2014). A chemical network is developed for the study comprising of all feasible chemical pathways 
Table 3 The set of atoms, ions, molecules, and molecular clusters important for the study (Sarangi and Cherchneff 2013, 2015; Biscaro and Cherchneff 2014, 2016)

\begin{tabular}{lllllll}
\hline Elements & & & & & & \\
\hline $\mathrm{He}$ & $\mathrm{C}$ & $\mathrm{N}$ & $\mathrm{O}$ & $\mathrm{Ne}$ & $\mathrm{Mg}$ & $\mathrm{Al}$ \\
$\mathrm{Si}$ & $\mathrm{S}$ & $\mathrm{Ar}$ & $\mathrm{Ti}$ & $\mathrm{Fe}$ & $\mathrm{Co}$ & $\mathrm{Ni}$ \\
Molecules & & & & & & \\
\hline $\mathrm{CO}$ & $\mathrm{CO}_{2}$ & $\mathrm{NO}$ & $\mathrm{O}_{2}$ & $\mathrm{MgO}$ & $\mathrm{AlO}$ & $\mathrm{SiO}$ \\
$\mathrm{SO}$ & $\mathrm{FeO}$ & $\mathrm{S}$ & $\mathrm{CN}$ & $\mathrm{SiC}$ & $\mathrm{CS}$ & $\mathrm{SiS}$ \\
$\mathrm{FeS}$ & $\mathrm{MgS}$ & $\mathrm{N}_{2}$ & & & & \\
Ions & & & & & & \\
\hline $\mathrm{He}^{+}$ & $\mathrm{C}^{+}$ & $\mathrm{N}^{+}$ & $\mathrm{O}^{+}$ & $\mathrm{Ne}^{+}$ & $\mathrm{Mg}^{+}$ & $\mathrm{Al}^{+}$ \\
$\mathrm{Si}^{+}$ & $\mathrm{S}^{+}$ & $\mathrm{Ar}^{+}$ & $\mathrm{Fe}^{+}$ & $\mathrm{CO}^{+}$ & $\mathrm{SiO}^{+}$ & $\mathrm{SO}^{+}$ \\
$\mathrm{O}_{2}{ }^{+}$ & $\mathrm{N}_{2}{ }^{+}$ & $\mathrm{C}_{2}{ }^{+}$ & & & &
\end{tabular}

Molecular clusters

\begin{tabular}{|c|c|c|c|c|c|c|}
\hline $\mathrm{C}_{2}$ & $\mathrm{C}_{3}$ & $\mathrm{C}_{4}$ & $\mathrm{C}_{5}$ & $\mathrm{C}_{6}$ & $\mathrm{C}_{7}$ & $\mathrm{C}_{8}$ \\
\hline $\mathrm{C}_{9}$ & $\mathrm{C}_{10}$ & $\mathrm{C}_{11}$ & $\mathrm{C}_{12}$ & $\mathrm{C}_{13}$ & $\mathrm{C}_{14}$ & $\mathrm{C}_{15}$ \\
\hline $\mathrm{C}_{16}$ & $\mathrm{C}_{17}$ & $\mathrm{C}_{18}$ & $\mathrm{C}_{19}$ & $\mathrm{C}_{20}$ & $\mathrm{C}_{21}$ & $\mathrm{C}_{22}$ \\
\hline $\mathrm{C}_{23}$ & $\mathrm{C}_{24}$ & $\mathrm{C}_{25}$ & $\mathrm{C}_{26}$ & $\mathrm{C}_{27}$ & $\mathrm{C}_{28}$ & \\
\hline$(\mathrm{SiO})_{2}$ & $\mathrm{Si}_{2} \mathrm{O}_{3}$ & $(\mathrm{SiO})_{3}$ & $\mathrm{Si}_{3} \mathrm{O}_{4}$ & $(\mathrm{SiO})_{4}$ & $\mathrm{Si}_{4} \mathrm{O}_{5}$ & $(\mathrm{SiO})_{5}$ \\
\hline $\mathrm{MgSi}_{2} \mathrm{O}_{3}$ & $\mathrm{MgSi}_{2} \mathrm{O}_{4}$ & $\mathrm{Mg}_{2} \mathrm{Si}_{2} \mathrm{O}_{4}$ & $\mathrm{Mg}_{2} \mathrm{Si}_{2} \mathrm{O}_{5}$ & $\left(\mathrm{MgSiO}_{3}\right)_{2}$ & & \\
\hline $\mathrm{Mg}_{3} \mathrm{Si}_{2} \mathrm{O}_{6}$ & $\mathrm{Mg}_{3} \mathrm{Si}_{2} \mathrm{O}_{7}$ & $\mathrm{Mg}_{4} \mathrm{Si}_{2} \mathrm{O}_{7}$ & $\left(\mathrm{Mg}_{2} \mathrm{SiO}_{4}\right)_{2}$ & & & \\
\hline $\mathrm{Al}_{2}$ & $(\mathrm{AlO})_{2}$ & $(\mathrm{AlO})_{3}$ & $\mathrm{AlO}_{2}$ & $\mathrm{Al}_{2} \mathrm{O}$ & $\mathrm{Al}_{2} \mathrm{O}_{3}$ & \\
\hline$\left(\mathrm{Al}_{2} \mathrm{O}_{3}\right)_{2}$ & $\left(\mathrm{Al}_{2} \mathrm{O}_{3}\right)_{3}$ & $\left(\mathrm{Al}_{2} \mathrm{O}_{3}\right)_{4}$ & & & & \\
\hline $\mathrm{Si}_{2}$ & $\mathrm{Si}_{3}$ & $\mathbf{S i}_{4}$ & $\mathrm{Mg}_{2}$ & $\mathrm{Mg}_{3}$ & $\mathrm{Mg}_{4}$ & \\
\hline $\mathrm{Fe}_{2}$ & $\mathrm{Fe}_{3}$ & $\mathrm{Fe}_{4}$ & $(\mathrm{FeS})_{2}$ & $(\mathrm{FeS})_{3}$ & $(\mathrm{FeS})_{4}$ & \\
\hline$(\mathrm{MgS})_{2}$ & $(\mathrm{MgS})_{3}$ & $(\mathrm{MgS})_{4}$ & $(\mathrm{SiC})_{2}$ & $(\mathrm{SiC})_{3}$ & $(\mathrm{SiC})_{4}$ & \\
\hline$(\mathrm{MgO})_{2}$ & $(\mathrm{MgO})_{3}$ & $(\mathrm{MgO})_{4}$ & $(\mathrm{FeO})_{2}$ & $(\mathrm{FeO})_{3}$ & $(\mathrm{FeO})_{4}$ & \\
\hline
\end{tabular}

relevant to the hot and dense environments. The network consists of about 600 reactions of different kinds in the gas phase. They can be further categorized into thermal (termolecular or 3-body processes, thermal fragmentation, bimolecular reactions, ion-molecule reactions, radiative association etc.) and non-thermal processes (photon induced, Compton-electron induced etc.) (Cherchneff and Dwek 2009). The reactions are characterized by the respective reactions rates. Hence the temporal variation of number density of a certain species $i$ located at mass zone $M_{r}$ in the ejecta at a certain time $t$, is given by,

$$
\begin{gathered}
\frac{\partial n_{i}\left(M_{r}, t\right)}{\partial t}=P_{i}-L_{i}=\sum_{j} k_{j i} n_{j} n_{i}-\sum_{k} k_{i k} n_{i} n_{k} \\
k_{i j}(T)=A_{i j} \times\left(\frac{T}{T_{0}}\right)^{v} \times \exp \left(-E_{i j} / T\right),
\end{gathered}
$$

where $P_{i}$ represents the sum over the all the production (formation) processes whereas $L_{i}$ represents the sum over all the destruction processes. The term $k_{i j}$ represents the tempera- 
Fig. 16 The nucleation scheme for silicates leading to the formation of the enstatite and forsterite dimers, which act as the seed in the condensation formalism. The reactant species are given on the side of the directional arrows in the schematic diagram (Goumans and Bromley 2012; Zachariah and Tsang 1993)

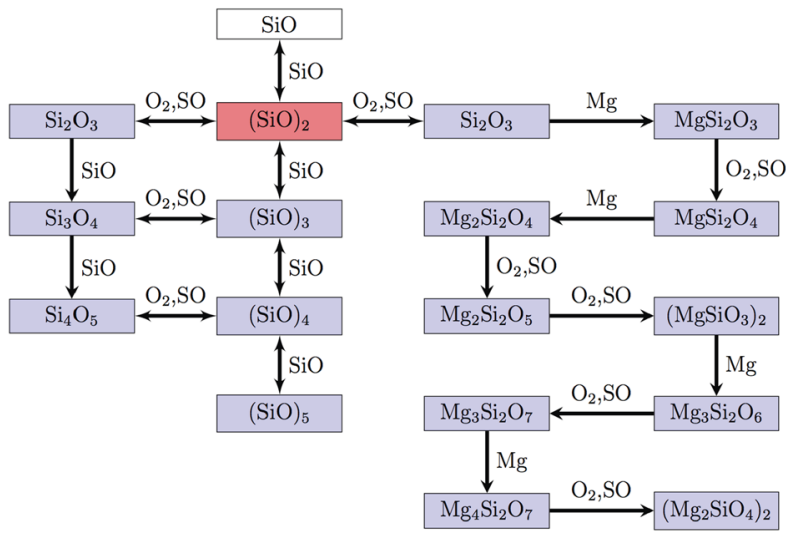

ture dependent reaction rates between the $i$ th and $j$ th species (Cherchneff and Dwek 2009). $T_{0}$ is a reference temperature chosen as $300 \mathrm{~K}$ for the study. $A_{i j}$ is the Arrhenius rate coefficient expressed in $\mathrm{s}^{-1}, \mathrm{~cm}^{3} \mathrm{~s}^{-1}$ or $\mathrm{cm}^{6} \mathrm{~s}^{-1}$ depending on the order of reaction and $E_{i j}$ is the activation energy in the unit of $\mathrm{K}^{-1}$. The factor $v$ represents the time dependence of the pre-exponential factor of the general Arrhenius equation. Typical values of the $v$ factor range between -1 to 1 . The gas phase chemistry is overall controlled by $N$ number of stiff coupled nonlinear ordinary differential equations similar to (2), where $N$ is the number of species that has been included in the study. This entire set of differential equations is solved simultaneously and the time evolution of the abundances for each species is estimated. The reaction rates are either theoretically calculated using translation theory or estimated in the laboratory. Standard chemical databases such as NIST, KIDA, UMIST, etc., are used as sources for the compilation of the network. The rate coefficients for the non-thermal processes are estimated using the rate of deposition of radioactive energy by the thermalized $\gamma$-rays in the ejecta (Cherchneff and Dwek 2009; Liu and Dalgarno 1994, 1995). The branching ratio between the energy deposited by electron through excitation, ionisation $\left(A \rightarrow A^{+}+e^{-}\right)$and fragmentation $\left(A B \rightarrow A+B^{+}+e^{-}\right)$depends on the mean energy per ion pair ( $W_{d}=E_{p} X_{i} / N_{d}$, where $N_{d}$ and $X_{i}$ are the number of ion pairs produced by the species in this process and the abundance of that species in the ejecta). The detailed account of all the non-thermal processes related to SN ejecta can be found in Cherchneff and Dwek (2009), Liu and Dalgarno (1995) \& Clayton et al. (1999).

A stochastic, kinetically driven approach has been adopted to study the nucleation of gas molecules to small clusters. The vaporisation experiments in the laboratory and the study of ceramic synthesis in the flames provide insights into similar nucleation processes under nonsteady state environment. The important species that demand detailed analysis are oxygen rich clusters of magnesium silicates, alumina and $\mathrm{Si}_{m} \mathrm{O}_{n}$ molecules, carbon chains, rings and fullerenes, silicon carbide, metallic sulphides and oxides, and pure metallic clusters. The upper limit of the cluster size in the nucleation network is not defined very specifically. The geometry of the molecules is the backbone to determine the final product of nucleation and the dust precursor in the network (Table 3 in bold font). The clusters that attain a three dimensional structure like a cage or twisted rings, has a larger collision cross section for coagulation (Cherchneff and Dwek 2010; Sarangi and Cherchneff 2015). Further, they are energetically more stable, and hence likely to be resilient under the destruction processes in the gas phase. In some cases, the favoured chemical routes for nucleation network are derived from quantum chemical calculations. Figure 17 presents the structures of a few stable 
Fig. 17 (1) $(\mathrm{SiO})_{5}$ (Reber et al. 2008), (2) $\left(\mathrm{MgSiO}_{3}\right)_{2}$ (Goumans and Bromley 2012), (3) $\mathrm{C}_{28}$ (Makurin et al. 2001), (4) $\mathrm{Mg}_{4}$ (Cherchneff and Dwek 2010), (5) $\left(\mathrm{Al}_{2} \mathrm{O}_{3}\right)_{2}$ (Gobrecht et al. 2016, private communications)
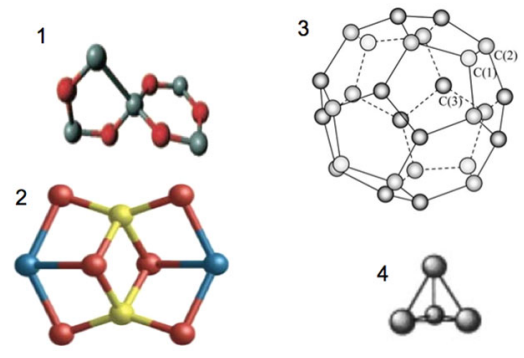

5

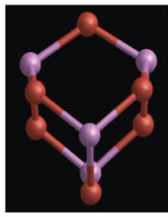

gas phase molecular clusters, which acts as precursor seeds to the condensation phase. The nucleation schemes vary vastly depending on the type of species. Figure 16 is a schematic presentation of the nucleation pathways that lead to stable $(\mathrm{SiO})_{n}$ compounds and magnesium silicates $\left(\mathrm{Mg}_{2} \mathrm{SiO}_{4}\right)_{2}$. In a hydrogen free environment, pure carbon chains are formed of sp-hybridization up to $\mathrm{C}_{9}$ followed by monocyclic and fullerenes. Cluster beam experiments suggest, the smallest stable structure of fullerene present in carbon vapour is $\mathrm{C}_{28}$ (Dunk et al. 2012). All the possible nucleation networks relevant for such environments are described in Cherchneff and Dwek (2009) \& Sarangi and Cherchneff (2013).

The model predicts the formation of several diatomic molecules in the ejecta, such as $\mathrm{CO}, \mathrm{SiO}, \mathrm{SO}, \mathrm{O}_{2}, \mathrm{CS}, \mathrm{SiS}, \mathrm{N}_{2}$ etc., over a span of a few (between 1 and 4) years after the explosion. The epoch of synthesis and the abundances vary with the zones in the ejecta. The findings are congruent with the observations of CO (Danziger et al. 1988; Catchpole et al. 1988) and $\mathrm{SiO}$ (Roche et al. 1991; Kotak et al. 2005, 2009) molecules in local supernovae, mainly SN1987A. Figure 18 shows the time evolution of $\mathrm{CO}$ and $\mathrm{SiO}$ masses in a $15 \mathrm{M}_{\odot}$ progenitor SN ejecta compared to the observations. $\mathrm{CO}$ molecules are essentially synthesized in the $\mathrm{C} / \mathrm{O}$ rich zone 4 , followed by the central region on the O-core (zone 2). $\mathrm{CO}$ formation follows very efficient chemical routes. Hence, the abundance increases as early as 150 days and becomes constant in mass by 700 days post-explosion. Due to the strong chemical bonds, once they are formed in the ejecta, the $\mathrm{CO}$ molecules are found to withstand the harsh environment conditions and possible destruction mechanisms. The chemical pathways important for $\mathrm{CO}$ formation are mainly: $\mathrm{C}+\mathrm{O}_{2} \rightarrow \mathrm{CO}+\mathrm{O}, \mathrm{C}+\mathrm{O} \rightarrow \mathrm{CO}+h v$ etc. The final masses estimated by the model are in accord with the findings of Kamenetzky et al. (2013) and also it matches well at $\sim 300$ days $\left(4 \times 10^{-3} \mathrm{M}_{\odot}\right)$ with the IR observations of SN1987A. There is no evidence of depletion of CO molecules with time. Hence it can be safely claimed that $\mathrm{CO}$ does not act as a precursor to the carbon dust (Sarangi and Cherchneff 2013; Biscaro and Cherchneff 2014).

$\mathrm{SiO}$ molecules are formed mainly in the central zones of the O-core, named as zones $1 \mathrm{~B}$, $2 \& 3$. The $\mathrm{SiO}$ increases rapidly to $8 \times 10^{-3} \mathrm{M}_{\odot}$ at day 200 from explosion in these regions. Reactions with $\mathrm{CO}, \mathrm{O}_{2}$ and radiative association of $\mathrm{Si}$ and $\mathrm{O}$ are the dominant chemical processes for the formation of $\mathrm{SiO}$ in the ejecta (Sarangi and Cherchneff 2013). Unlike CO, the rapid formation $\mathrm{SiO}$ molecules in the ejecta is subsequently also followed by decline of the abundances after day $\sim 300$. This is attributed to the rapid conversion into small clusters, which can be correlated to the IR observations of SN2004et and SN1987A (Kotak et al. 2009).

Table 4 summarises the final masses of all the important molecules, small clusters and residual dust precursors in the gas phase, at day 2000 post-explosion. Even though $\mathrm{O}_{2}$ is the most abundant molecule in the ejecta, due to symmetry and zero dipole moment, the vibrational transitions in the IR are absent. The model further predicts the presence of SO and $\mathrm{SiS}$ in the gas phase, where the presence of SO is most likely (Matsuura et al. 2017), while 
Table 4 Masses $\left(\mathrm{M}_{\odot}\right)$ of molecules, intermediate clusters and uncondensed dust-clusters in the gas phase at 2000 days post-explosion present in the SN ejecta of a $15 \mathrm{M}_{\odot}$ progenitor (Sarangi and Cherchneff 2013, 2015) are tabulated

\begin{tabular}{|c|c|c|c|c|c|c|c|c|c|}
\hline Zones & $\mathrm{Z} 1 \mathrm{~A}$ & $\mathrm{Z1B}$ & $\mathrm{Z} 2$ & $\mathrm{Z3}$ & $\mathrm{Z} 4 \mathrm{~A}$ & Z4B & $\mathrm{Z} 5$ & Z6 & Total \\
\hline & \multicolumn{9}{|c|}{ Molecules } \\
\hline Mass & $9.6(-2)$ & $9.5(-2)$ & 0.292 & 0.347 & 0.195 & 0.225 & 0.75 & 0.347 & 2.35 \\
\hline $\mathrm{SiO}$ & $2.0(-8)$ & $1.3(-8)$ & $1.3(-8)$ & $2.1(-9)$ & $\ldots$ & $\cdots$ & $\cdots$ & $\cdots$ & $4.8(-8)$ \\
\hline $\mathrm{O}_{2}$ & $\ldots$ & $2.8(-5)$ & 0.15 & 0.16 & $6.2(-2)$ & $4.9(-3)$ & $\ldots$ & $\cdots$ & 0.38 \\
\hline $\mathrm{CO}$ & $7.5(-7)$ & $1.7(-5)$ & $2.2(-3)$ & $6.6(-3)$ & $9.5(-2)$ & 0.14 & $2.9(-3)$ & $\ldots$ & 0.25 \\
\hline SO & $\ldots$ & $1.5(-2)$ & $3.8(-3)$ & $1.0(-4)$ & $1.1(-4)$ & $\ldots$ & $\ldots$ & $\ldots$ & $1.9(-2)$ \\
\hline $\mathrm{SiS}$ & $4.3(-2)$ & $2.1(-7)$ & $\ldots$ & $\ldots$ & $\ldots$ & $\ldots$ & $\ldots$ & $\ldots$ & $4.3(-2)$ \\
\hline $\mathrm{CO}_{2}$ & $\cdots$ & $\cdots$ & $1.7(-5)$ & $2.7(-5)$ & $\cdots$ & $\cdots$ & $\cdots$ & $\cdots$ & $4.4(-5)$ \\
\hline $\mathrm{CS}$ & $\ldots$ & $\cdots$ & $\ldots$ & $\ldots$ & $\ldots$ & $\ldots$ & $1.5(-4)$ & $\ldots$ & $1.5(-4)$ \\
\hline $\mathrm{N}_{2}$ & $\cdots$ & $\cdots$ & $\cdots$ & $3.2(-7)$ & $3.5(-7)$ & $\cdots$ & $3.1(-3)$ & $\ldots$ & $3.1(-3)$ \\
\hline Total & $4.3(-2)$ & $1.5(-2)$ & 0.156 & 0.167 & 0.157 & 0.15 & $6.2(-3)$ & 0 & 0.695 \\
\hline \multirow[t]{2}{*}{ Efficiency (\%) } & 44.8 & 15.8 & 53.4 & 48.1 & 80.5 & 66.7 & 0.8 & 0 & 29.6 \\
\hline & \multicolumn{9}{|c|}{ Intermediate molecular clusters } \\
\hline $\mathrm{Si}_{m} \mathrm{O}_{n}$ & $\cdots$ & $5.5(-2)$ & $3.4(-2)$ & $2.9(-3)$ & $\cdots$ & $\cdots$ & $\cdots$ & $\cdots$ & $9.0(-2)$ \\
\hline $\mathrm{C}_{2 n}$ & $\cdots$ & $\cdots$ & $\cdots$ & $\cdots$ & $\cdots$ & $\cdots$ & $5.5(-3)$ & $\cdots$ & $5.5(-3)$ \\
\hline $\mathrm{Fe}_{n}$ & $8.2(-5)$ & $\cdots$ & $\cdots$ & $\cdots$ & $\cdots$ & $\cdots$ & $\cdots$ & $\cdots$ & $8.2(-5)$ \\
\hline \multirow[t]{2}{*}{ Total } & $8.2(-5)$ & $5.5(-2)$ & $3.4(-2)$ & $2.9(-3)$ & $\cdots$ & $\cdots$ & $5.5(-3)$ & $\cdots$ & $9.6(-2)$ \\
\hline & \multicolumn{9}{|c|}{ Uncondensed dust clusters } \\
\hline$\left(\mathrm{Mg}_{2} \mathrm{SiO}_{4}\right)_{2}$ & $\cdots$ & $9.9(-6)$ & $4.1(-5)$ & $1.7(-5)$ & $2.2(-6)$ & $2.0(-6)$ & $1.9(-5)$ & $\cdots$ & $9.1(-5)$ \\
\hline$\left(\mathrm{Al}_{2} \mathrm{O}_{3}\right)_{4}$ & $\cdots$ & $3.4(-6)$ & $4.8(-5)$ & $4.7(-5)$ & $\ldots$ & $\ldots$ & $\ldots$ & $\cdots$ & $9.9(-5)$ \\
\hline $\mathrm{C}_{28}$ & $\cdots$ & $\cdots$ & $\cdots$ & $\cdots$ & $\cdots$ & $\cdots$ & $1.0(-8)$ & $\cdots$ & $1.0(-8)$ \\
\hline $\mathrm{Mg}_{4}$ & $\cdots$ & $\cdots$ & $1.4(-5)$ & $1.8(-5)$ & $\cdots$ & $\cdots$ & $\cdots$ & $\cdots$ & $3.2(-5)$ \\
\hline $\mathrm{Si}_{4}$ & $9.7(-6)$ & $\cdots$ & $\cdots$ & $\cdots$ & $\cdots$ & $\cdots$ & $\cdots$ & $\cdots$ & $9.7(-6)$ \\
\hline $\mathrm{Fe}_{4}$ & $6.5(-6)$ & $\cdots$ & $\cdots$ & $\cdots$ & $\cdots$ & $\cdots$ & $\cdots$ & $\cdots$ & $6.5(-6)$ \\
\hline $\mathrm{SiC}_{4}$ & $\cdots$ & $\cdots$ & $\cdots$ & $\cdots$ & $\cdots$ & $\cdots$ & $1.1(-5)$ & $\cdots$ & $1.1(-5)$ \\
\hline $\mathrm{FeS}_{4}$ & $2.2(-7)$ & $\cdots$ & $\cdots$ & $\cdots$ & $\cdots$ & $\cdots$ & $\cdots$ & $\cdots$ & $2.2(-7)$ \\
\hline Total & $1.6(-4)$ & $1.3(-5)$ & $1.0(-4)$ & $8.2(-5)$ & $2.2(-6)$ & $2.0(-6)$ & $3.0(-5)$ & $\cdots$ & $2.5(-4)$ \\
\hline
\end{tabular}

SiS yet to be confirmed from observations. Small clusters such as $\left(\mathrm{Mg}_{2} \mathrm{SiO}_{4}\right)_{2},\left(\mathrm{Al}_{2} \mathrm{O}_{3}\right)_{4}$, or $\mathrm{C}_{28}$ fullerenes are stable gas-phase monomers of dust; therefore they undergo quick depletion immediately after synthesis. Other than that, there are a few intermediate clusters of the form $\mathrm{Si}_{n} \mathrm{O}_{m}$ which remain in the ejecta as uncondensed residue. About $30 \%$ of all the gas in the ejecta is in molecular form after a few years of the explosion.

\subsubsection{Condensation Phase}

The dust precursors act as seeds in the condensation scheme, where they grow by coagulation and coalescence to form large grains. The gas phase reactions and grain condensation occur in tandem. The model is designed to maintain the balance between gas phase 

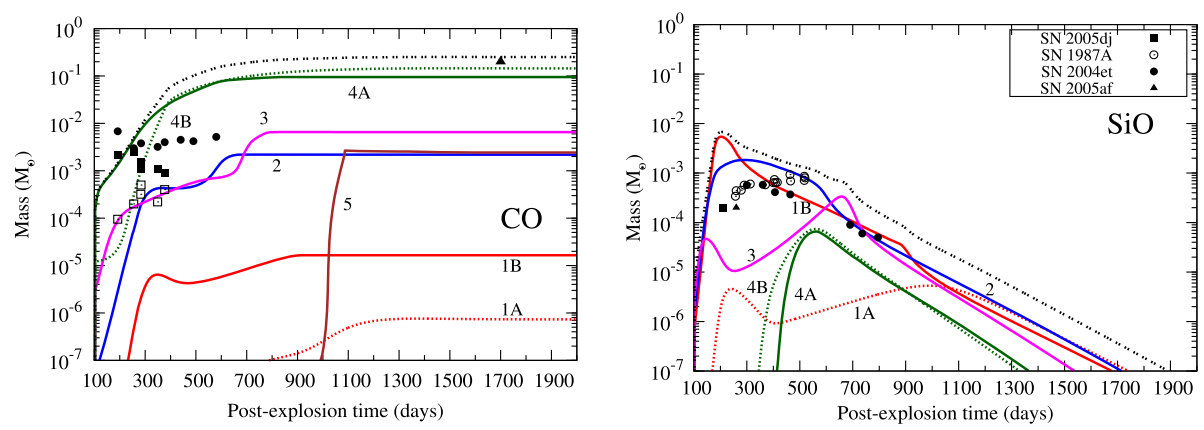

Fig. 18 Left-panel: Mass of $\mathrm{CO}$ in a $15 \mathrm{M}_{\odot}$ model presented in $\mathrm{M}_{\odot}$ as a function of various ejecta zones as labelled by the side of the lines. The black dotted line corresponds to the total over all the zones. CO masses derived from the observations of SN1987A are shown as, LTE: open squares, non-LTE: squares (Liu et al. 1992), thermal assumption: circles (Liu and Dalgarno 1995), ALMA data: triangle (Kamenetzky et al. 2013). The ALMA data is not true to the time axis, but rather indicates the final mass at late times as compared to the model results. Right panel: Evolution of $\mathrm{SiO}$ mass $\left(\mathrm{M}_{\odot}\right)$ with post-explosion time in days as a function of different zones in the ejecta. The black dotted black line corresponds to the sum over the SiO mass from all the zones. The solid square, circle, and triangle indicates the mass of SiO reported from observations in IR from several supernovae as specified in the legend box (Sarangi and Cherchneff 2013)

processes, the solid phase interactions and the smooth transition between the two interactive phases at all times. The formalism has been developed by Jacobson (2005), where the variation of the grain number density of volume $v$ is described by the integro-differential coagulation equation,

$$
\frac{d n_{v}(t)}{d t}=\frac{1}{2} \int_{0}^{v} \beta_{v-v^{\prime}, v} n_{v-v^{\prime}} n_{v^{\prime}} d v^{\prime}-n_{v} \int_{0}^{\infty} \beta_{v, v^{\prime}} n_{v^{\prime}} d v^{\prime}
$$

where $\beta$ is the rate coefficient of coagulation between particles. The rate coefficient is controlled by physical processes such as Brownian diffusion, convective Brownian motion enhancement, gravitational collection, turbulent inertial motion and Van der Waals forces. In free molecular regimes (where mean free-path of a particle in the gas is much larger compared to the mean radius of a grain), Brownian diffusion prevails as the most important phenomena along with Van der Waals interactions. Brownian diffusion accounts for the scattering, collision and coalescence of the grains through Brownian motion. On the other hand, the Van der Waals forces develop weak, local charge fluctuations that enhance the rate of coagulation for particles with sizes in the molecular range. Solving the appropriate equations, the concentrations and the grain size distribution function for individual dust types are estimated. This formalism has been used to model dust in SN ejecta and winds of AGB stars by Sarangi and Cherchneff (2015), Biscaro and Cherchneff (2016) \& Gobrecht et al. (2016).

The dust components comprise of silicates, alumina, carbon, iron sulphide, silicon carbide and pure metallic clusters of magnesium, iron and silicon. The analysis traces the evolution mechanism of all these dust components individually. The formation of dust grains in the ejecta is found to be highly sensitive to the gas densities and the concentration of the specific clusters in consideration. Hence, the dust formation scenario varies to a great extent from one zone to the other even for a single dust type. The estimated mass of each dust component and the size distributions of the grains were calculated as a function of post-explosion time. There is no specific threshold in size to differentiate between the large 
Fig. 19 Dust masses for grains with radius larger than $10 \AA$ summed over all ejecta zones as a function of post-explosion time and dust type. The dotted black line corresponds to the total dust mass formed in the homogeneous ejecta with progenitor mass $15 \mathrm{M}_{\odot}$ (Sarangi and Cherchneff 2015)

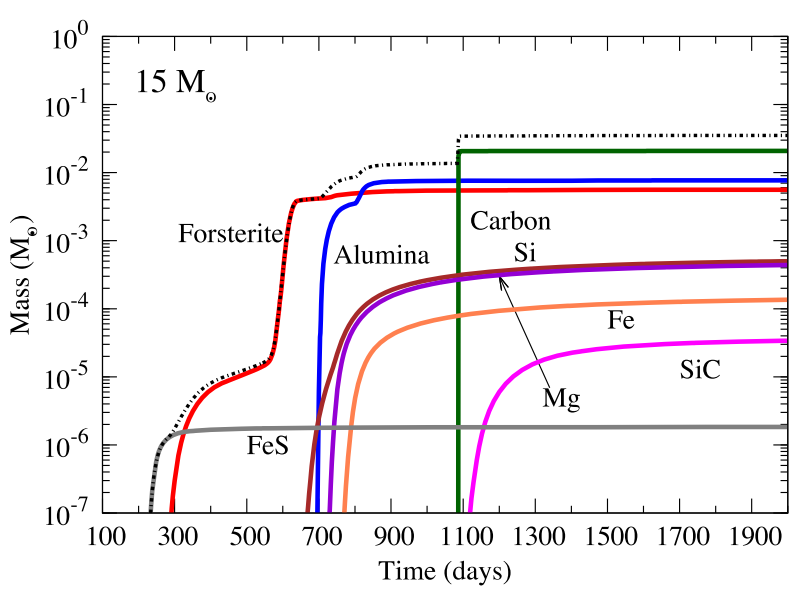

Table 5 Dust masses $\left(\mathrm{M}_{\odot}\right)$ at different post-explosion epochs for the $15 \mathrm{M}_{\odot}$ progenitor model. The final peak grain size of each dust type is also indicated in the table (Sarangi and Cherchneff 2015)

\begin{tabular}{lllllllll}
\hline Dust type & \multicolumn{9}{l}{ Post-explosion time (days) } & & & & & $a_{\text {peak }}(\AA)$ \\
\hline & 500 & 700 & 900 & 1100 & 1200 & 1500 & 2000 & \\
Forsterite & $1.1(-5)$ & $4.2(-3)$ & $5.3(-3)$ & $5.5(-3)$ & $5.5(-3)$ & $5.6(-3)$ & $5.6(-3)$ & $64 ; 168$ \\
Alumina & - & $6.1(-6)$ & $7.4(-3)$ & $7.6(-3)$ & $7.6(-3)$ & $7.7(-3)$ & $7.7(-3)$ & 96 \\
Carbon & - & - & - & $2.0(-2)$ & $2.0(-2)$ & $2.0(-2)$ & $2.1(-2)$ & 134 \\
Pure Magnesium & - & $2.5(-6)$ & $1.9(-4)$ & $3.2(-4)$ & $3.6(-4)$ & $4.4(-4)$ & $5.0(-4)$ & 29 \\
Pure Silicon & - & - & $1.5(-4)$ & $2.7(-4)$ & $3.1(-4)$ & $3.8(-4)$ & $4.4(-4)$ & 69 \\
Pure Iron & - & - & $4.1(-5)$ & $9.1(-5)$ & $9.4(-5)$ & $1.2(-4)$ & $1.4(-4)$ & 36 \\
Silicon Carbide & - & - & - & - & $6.1(-6)$ & $2.6(-5)$ & $3.4(-5)$ & 9 \\
Iron Sulphide & $1.7(-6)$ & $1.8(-6)$ & $1.8(-6)$ & $1.8(-6)$ & $1.8(-6)$ & $1.8(-6)$ & $1.8(-6)$ & 21 \\
Total & $1.3(-5)$ & $4.3(-3)$ & 0.013 & 0.034 & 0.034 & 0.035 & 0.035 & \\
\hline
\end{tabular}

molecular clusters and the dust particles. From the observational point of view, the dust formation in the ejecta is characterised by an excess in IR spectral energy distribution and blue shift of optical emission lines. From the modeling perspective, a fiducial size of $10 \AA$ has been chosen as the minimum size of grains in the ejecta, which can be categorised as dust. The grains smaller in size are considered as uncondensed molecular clusters, which remain in gas phase. Figure 19 illustrates the evolution of dust masses of all the dust components from day 100 to 2000 after explosion. The masses of dust components in the figure are presented as the sum over all the ejecta zones.

Formation of dust in the ejecta dawns after 200 days post-explosion time with the formation of iron sulphide grains. Later on in the period of 300-400 days silicates (forsterite) starts to form mainly in the inner zones of the O-core. The mass of dust surges up at $\sim 550$ days due to synthesis of silicates in the central zone 2 . The process of gradual increase of dust mass continue with the advent of alumina at day 800 and lately by amorphous carbon at 1100 days. This way the dust mass increases from $10^{-5} \mathrm{M}_{\odot}$ at 400 days to $0.035 \mathrm{M}_{\odot}$ at the end of four years. Table 5 presents all the evolution pattern of each dust type over a span of a 2000 days after explosion. Amorphous carbon, alumina and forsterite are the most abun- 
Fig. 20 The final size distribution $f(a)$ of dust grains at 2000 days post-explosion is presented for all the dust components (Sarangi and Cherchneff 2015). The distribution function is expressed in terms of total number of particles per unit radius $\left(N_{\text {tot }}(a) . \AA^{-1}\right)$

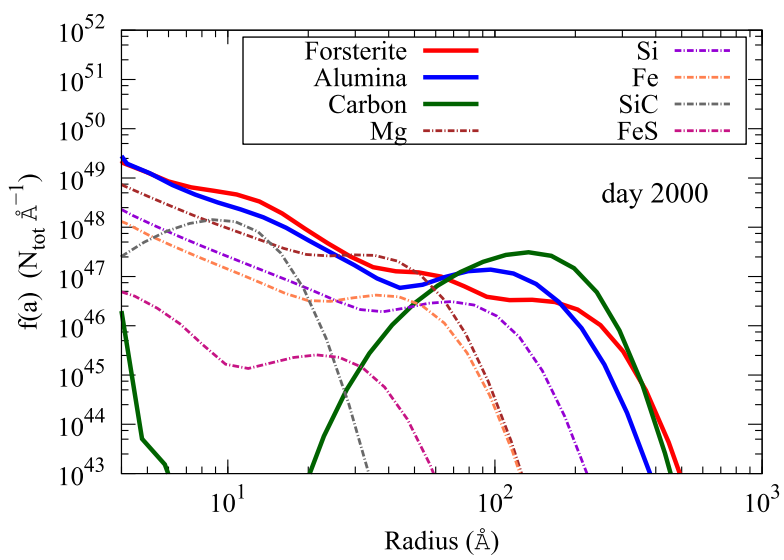

dant dust types in the ejecta. The range of dust masses closely agrees with the estimated masses of dust derived from observational data in the IR. At times between 200-600 days, the mid-IR observations predict the presence of dust in the ejecta with masses typically in the order of $10^{-5}$ to $10^{-3} \mathrm{M}_{\odot}$ (Kotak et al. 2009, 2005; Elmhamdi et al. 2003b; Wooden et al. 1993; Inserra et al. 2011; Sugerman et al. 2006; Gallagher et al. 2012). These findings are consistent with the model results of the total dust mass over time (Fig. 19). Submm observations however report the presence of much larger masses of dust in the remnants. In Cas $\mathrm{A}, \sim 0.08 \mathrm{M}_{\odot}$ of dust is inferred from Herschel data (Barlow et al. 2010). In case of SN1987A, Matsuura et al. 2011 has derived masses of dust in the range of $0.4-0.7 \mathrm{M}_{\odot}$ to be present in the ejecta after 28 years from explosion. Recent ALMA observation concluded the final dust mass to be about $0.5 \mathrm{M}_{\odot}$ (Matsuura et al. 2015). Also for the filaments of Crab nebula an estimated mass of $0.1-0.2 \mathrm{M}_{\odot}$ is required to fit the Herschel data as explained by Gomez et al. 2012. The final mass of dust estimated in this study ends up in $0.035 \mathrm{M}_{\odot}$, and we can suppose the dust mass will remain consistent till the advent of reverse shock in the remnant. These trends derived from the model match well with the results of submm observations (Sarangi and Cherchneff 2015; Biscaro and Cherchneff 2014). However the final masses reported from these late time observational data are 2-10 times larger than our assessment. Importantly though, all the masses reported from observations are derived by fitting the spectral energy distributions at IR and submm wavelengths where some composition of dust has been assumed. The dust masses from observations hence can be sensitive to the choice of dust components, their compositions and size at $a \ll \lambda$. Finally, there are several options for the choice of optical constants related to each dust type, and the results vary significantly from one to the other. In most of the cases, the size distributions were assumed to either follow a power law profile like the standard Mathis-Rumpl-Nordsieck (MRN) distribution (Mathis et al. 1977) or the grains were chosen to be of identical sizes. All these factors add to some uncertainty in the estimation of dust masses fitting the observed spectra.

The size distribution function defines the number of grains per unit of particle radius, assuming a spherical geometry. The size distribution function $f(a)$, for all the important dust species at day 2000 is presented in Fig. 20. The main three dust components carbon, alumina and forsterite are presented in solid colours in the figure. The distribution function at a given time depends on (a) the number of monomers present in the gas phase, (b) the rate of coagulation at the given temperature and (c) the concentration of grains from the previous time step. The prime dust components being more abundant, also grow to form larger grains in the order of 200-500 $\mathrm{A}$. The size distribution function of one dust species 

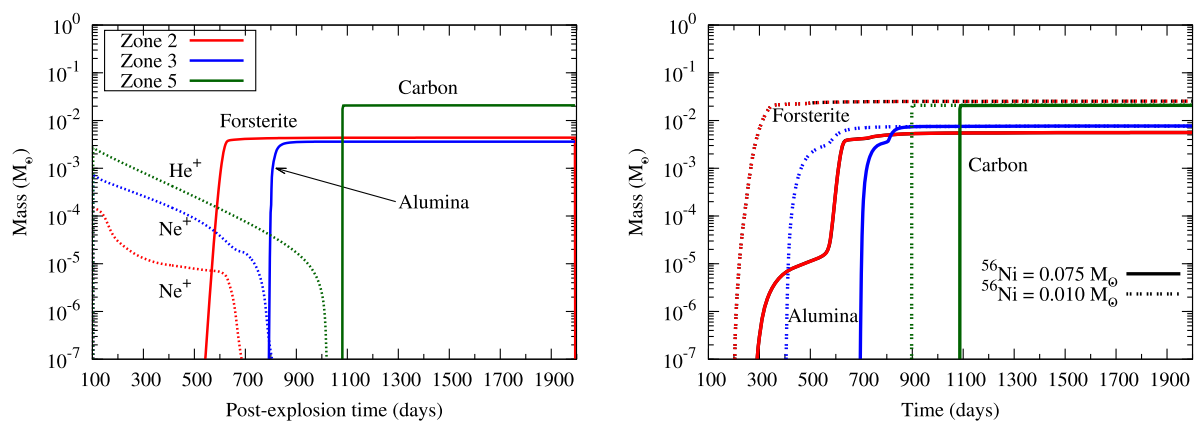

Fig. 21 Left-panel: The figure presents the correlation between the ions of the nobel gases and the epochs of dust formation. The presence of $\mathrm{He}^{+}, \mathrm{Ne}^{+}$or $\mathrm{Ar}^{+}$ions in the gas tend to hinder the dust formation processes in the respective ejecta zones, as illustrated in the figure and the text. Right panel: The time evolution of forsterite, alumina and carbon dust masses for standard $\left({ }^{56} \mathrm{Ni}=0.075 \mathrm{M}_{\odot}\right)$ model and a lower ${ }^{56} \mathrm{Ni}$ mass $\left({ }^{56} \mathrm{Ni}=0.01 \mathrm{M}_{\odot}\right)$ model are compared in this figure (Sarangi and Cherchneff 2013 )

differs in behaviour from the other and hence cannot be defined by a single mathematical expression. Depending on the formation zones in the ejecta, the distribution functions can have a single peak or sometimes are found to have multiple peaks. The findings suggest that the dust masses reside mainly in the larger grains even though the population of large grains may be less than that of relatively smaller grains in the ejecta. Moreover, the condensation phase is an efficient process in the ejecta and once the large grains are formed, they are unlikely to segregate to smaller size fragments.

The size distributions of dust produced by SNe do not follow a MRN size variation both for single dust type and for the total dust distribution. The size distributions derived from the study have less small grains with radius $a<30 \AA$ and are skewed towards large grains in the size range $40-1000 \AA$, compared with the MRN distribution. This result clearly indicates that the use of a MRN size distribution for modeling the fluxes from a SN ejecta is inappropriate. Further, using MRN for dust sputtering calculations in the SNe remnants may lead to erroneous results on the derived dust masses and the dust mass that survives shock processing in the remnants. Ideally if there at all exists a distribution function for supernovae dust grains, that should be defined by the dust size patterns of a single dust component in a given zone, and not over a sum of all the species. The analysis clearly indicates none of the dust components follow a power law pattern of dust sizes at any epoch.

The ions of the nobel gases, namely helium, neon and argon present in the ejecta, play an important role in moulding the chemistry (Lepp et al. 1990). The $\gamma$-rays in the ejecta produced by radioactivity undergo Compton scattering and degrade to X-rays. The energetic Compton electrons further lose energy by excitation and ionisation of the gas. By day 100, most of the atoms in the ejecta gets ionised to their corresponding cation through collision with the fast Compton electrons. The ground state energy level of their inert parents being very low, the cations of $\mathrm{He}, \mathrm{Ne}$ and Ar actively participate in charge exchange processes with newly synthesised molecules, leading to their destruction (e.g. $\mathrm{AB}+\mathrm{He}^{+} \rightarrow \mathrm{A}+\mathrm{B}^{+}+\mathrm{He}$ ). The impact is clearly evident in the outermost zones of the He-core, where the detrimental effects of $\mathrm{He}^{+}$delay the appearance of stable molecules and clusters in the ejecta till $\sim$ day 1000. Figure 21 (left-panel) presents the correlation between the epoch of dust formation to the abundances of $\mathrm{He}^{+}, \mathrm{Ne}^{+}$or $\mathrm{Ar}^{+}$in the ejecta zones. With the passage of time, effect of radioactivity decreases as most of the ${ }^{56} \mathrm{Co}$ already gets converted to stable ${ }^{56} \mathrm{Fe}$. The energy from the $\gamma$-rays and X-rays get deposited in the ejecta and a small fraction escapes 
outside the core. Thereafter, the abundance of Compton electrons also falls rapidly and all the cations eventually recombine back to their stable inert parents. The molecule and dust synthesis gain in efficiency when the ions of $\mathrm{He}, \mathrm{Ne}$ and $\mathrm{Ar}$ are no longer present in the ejecta.

The amount of radioactive elements produced by the explosion also directly influences the chemical yields. The radioactive ${ }^{56} \mathrm{Ni}$ mass produced by a $\mathrm{SN}$ can be derived from the variation of the optical light curves and $\mathrm{H}_{\alpha}$ luminosities in the nebula phase (Elmhamdi et al. 2003a). Sarangi and Cherchneff (2013) provides a list of local SNe with their estimated progenitor masses as well as the masses of ${ }^{56} \mathrm{Ni}$ produced by the explosion. Observational findings support the trend derived by Hamuy (2003) that more massive supernovae produce more energetic explosions, and supernovae with greater energies produce larger ${ }^{56} \mathrm{Ni}$ masses. The energy deposited by the radioactive ${ }^{56} \mathrm{Ni}$ in the ejecta is linearly proportional to the its mass. Therefore, the rate coefficients for the destructions by energetic Compton electrons are directly connected to the mass of ${ }^{56} \mathrm{Ni}$. Physically, a smaller ${ }^{56} \mathrm{Ni}$ mass in the ejecta reduces the number of Compton electrons resulting from the degradation of a lower amount of $\gamma$-rays. Therefore, fewer ions such as $\mathrm{He}^{+}, \mathrm{Ne}^{+}$or $\mathrm{Ar}^{+}$are produced, thereby enhancing the rate of recombination. The destruction rates for molecules and clusters (e.g., $\mathrm{SiO}, \mathrm{C}_{2}$, $\mathrm{AlO}$ ) are much less severe for a low ${ }^{56} \mathrm{Ni}$ mass case, because of the lower $\mathrm{Ni}^{+}$and $\mathrm{He}^{+}$ concentration. So the chances of survival for the molecules and clusters increase, thereby also leading to early condensation of dust grains. Figure 21 (right-panel) shows the evolution of silicates, alumina and carbon dust masses corresponding to ${ }^{56} \mathrm{Ni}=0.075 \mathrm{M}_{\odot}$ and ${ }^{56} \mathrm{Ni}=$ $0.01 \mathrm{M}_{\odot}$ cases and compares the respective yields.

\section{Mixing and Clumpiness}

A homogeneous, 1D model of the ejecta is capable to indicate trends, which are closely similar to the actual event. Nevertheless, a real supernova ejecta is neither homogeneous nor spherically symmetric. Observational findings suggest the possibility of macroscopic mixing of ${ }^{56} \mathrm{Ni}$ in the outer layers of the H-He core (McCray 1993; Hanuschik et al. 1988). Detection of hard X-rays and $\gamma$-ray lines in SN1987A, immediately after the explosion, indicates a presence of a high-velocity component of radioactive ${ }^{56} \mathrm{Ni}$ (Dotani et al. 1987; Syunyaev et al. 1987; Matz et al. 1988). The mass of the high velocity ( $>3000 \mathrm{~km} / \mathrm{s}){ }^{56} \mathrm{Ni}$ clump is estimated to be about $10^{-3} \mathrm{M}_{\odot}$, which is between 4-17\% of the total ${ }^{56} \mathrm{Ni}$ produced at the explosion (Haas et al. 1990; Utrobin et al. 1995), as explained in Fig. 22 (Wongwathanarat et al. 2015). Therefore, the mixing of ${ }^{56} \mathrm{Ni}$ in the earliest days post-explosion is intense yet not complete. As a result, a two-phase $\mathrm{SN}$-ejecta structure is formed. Low-density blobs consisting of Fe-bubbles are created by the energy input from radioactive ${ }^{56} \mathrm{Co}$ and ${ }^{56} \mathrm{Ni}$, which is surrounded by compressed high density metal-rich ejecta (Blondin et al. 2001).

The multi-D explosion models study the presence of Rayleigh-Taylor unstable filaments in the non-homogeneous ejecta (Kifonidis et al. 2003, 2006; Joggerst et al. 2010; Hammer et al. 2010a; Wongwathanarat et al. 2015) of Type II supernovae. Figure 22 presents the isosurfaces of various metal-rich blobs after a few minutes post-explosion (Wongwathanarat et al. 2015). Moreover, the deviation from spherical symmetry is also evident from the structure of the ejecta.

The explosion energy is imparted in the ejecta in form of kinetic energy, which triggers an outward flow with velocities of around $2000-2500 \mathrm{~km} / \mathrm{s}$. If all the materials in the Hecore travel at a constant velocity, the density of the outflowing matter should decrease as 
Fig. 22 The isosurfaces of constant mass-fraction of $\mathrm{Ni}$ plumes and $\mathrm{O} / \mathrm{Mg}$ zone at $9000 \mathrm{~s}$ post outburst is shown in the Figure adopted from

Wongwathanarat et al. (2015). The ${ }^{56} \mathrm{Ni}$ blob is found to travel at velocities between 3800 and $4000 \mathrm{~km} / \mathrm{s}$
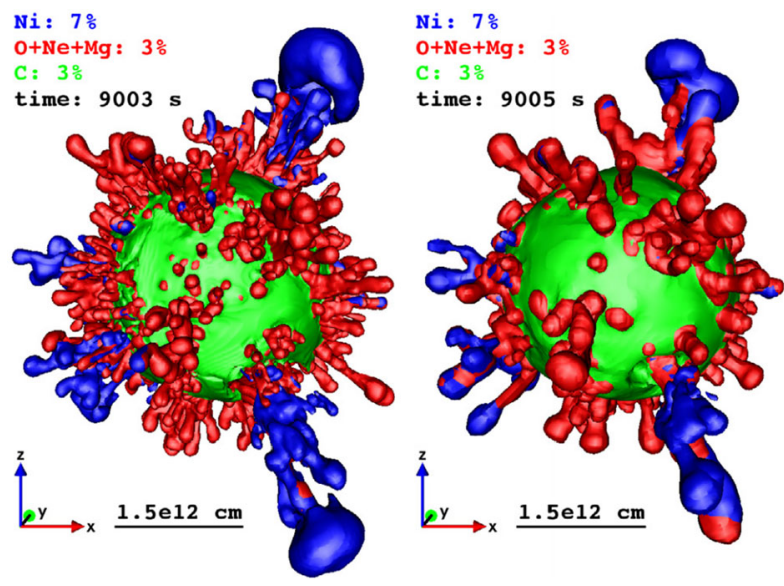

$t^{-2}$ law (Woosley 1988). However, the active radioactive processes (e.g. ${ }^{56} \mathrm{Ni} \rightarrow{ }^{56} \mathrm{Co} \rightarrow$ ${ }^{56} \mathrm{Fe}$ ) deposit the extra energy in the innermost Ni-Co core. Further, the neutrino-driven convection in the ejecta creates inhomogeneity between the layers (Joggerst et al. 2010). This creates a velocity shear across the zones of the ejecta, and the inner zone with extra energy tends to expand faster (Woosley 1988). The velocity shear induces the homologous expansion in the ejecta. Therefore, the fast moving low-density Ni-layers penetrate in the high density overlying zones of the ejecta. This density inversion occurs within a day of the explosion, thereby creating the Rayleigh-Taylor instabilities. The mixed Ni-bubbles further deposit energy in the O-core, which is in turn accelerated through the outer $\mathrm{He} / \mathrm{N} / \mathrm{C}$ zones. These instabilities lead to the fragmentation of the He-core, but the chemical stratification persists over time. Importantly though, this velocity shear results in clumping of the ejecta very early after the explosion.

Radiative transfer models trying to fit the IR contribution from the ejecta dust have repeatedly emphasized on the clumpy nature of the medium (Ercolano et al. 2007; Gallagher et al. 2012). Further, in order to account for the isotopic anomalies in the presolar grains in meteorites of $\mathrm{SN}$ origin, a small percentage of element mixing in microscopic scales is necessary. The scenario of mixing and inhomogeneity in the ejecta can only be modelled using hydrodynamic simulations, which is a very computationally rigorous process when all the important metals in the ejecta are being traced. Macroscopic mixing between the ejecta zones following the explosion is evident from observations and simulations. However, the possibility or the mechanism of microscopic diffusion of metals between the zone boundaries is not yet well understood.

A quasi-clumpy model based on SN1987A was formulated by Sarangi and Cherchneff (2015) using the volume filling factors for individual zones derived by Jerkstrand et al. (2011) through the study of the ultraviolet, optical, and near-IR emission lines. Dust formation is found to be faster and more efficient inside a high-density clump. However, depending on the optical depth of the clumpy region and rapid cooling of the newly formed grains, the dust can remain hidden and escape mid-IR detection. Microscopic mixing between inner $(\mathrm{Si} / \mathrm{S})$ and outer $(\mathrm{H} / \mathrm{He} / \mathrm{C})$ zones would lead to formation of SiC grains (important constituent in presolar grains) in larger abundance and H-bearing species such as recently detected $\mathrm{HCO}^{+}$(Matsuura et al. 2017). 


\section{Summary of Current State-of-the-Art}

Dust formation scenario in $\mathrm{SNe}$ is a complex interplay between several physical and chemical processes, which may vary depending on minimal alteration of conditions. Therefore, formulation of an all-inclusive single model of dust in core-collapse $\mathrm{SNe}$ most of the time over-simplifies the actual scenario. On the contrary, a more acceptable approach is to understand the general trends and then apply the knowledge to study any specific SN with its given constraints. Here we shall summarise the aspects related to the parameter space, which characterises the physics and chemistry of dust formation in a typical SN-ejecta.

(a) Core-collapse supernovae, mainly Type II-P, are the most efficient dust producers among all SN-types. On the other hand, the amount of dust formed in Type Ia SN is negligible.

(b) Dust formation in the ejecta is characterised by the chemistry prevailing in a non-steady state non-thermodynamic equilibrium environment.

(c) In a typical SN ejecta, dust formation commences within a year from the explosion and the dust mass continues to increase for a couple of years. The span depends essentially on the density and clumpiness of the medium.

(d) Dust synthesis is controlled by simultaneous processes of nucleation and condensation. Nucleation of stable dust clusters act as the bottleneck to the entire chain of chemical network. These molecular clusters resulting from nucleation determines the chemical composition of the dust as well as the earliest epochs of dust formation.

(e) Theory predicts of about $30-40 \%$ of the total mass in the ejecta. $\mathrm{CO}, \mathrm{SiO}, \mathrm{O}_{2}, \mathrm{CS}, \mathrm{AlO}$, $\mathrm{SO}$ etc. are important molecular species. Dust, on the other hand, is about $3-10 \%$ of the total ejecta mass.

(f) The dust budget in the $\mathrm{SN}$ ejecta is comprises of silicates, amorphous carbon, alumina and pure metallic clusters of silicon, iron and magnesium. There is also the possibility of mixed species as the coexisting grains in a certain zone might coagulate heterogeneously.

(g) Dust masses and grain sizes are inversely related to the amount of radioactive ${ }^{56} \mathrm{Ni}$ produced at the time of explosion. On the other hand, they are directly proportional to the degree of clumpiness.

(h) Relative abundances of C-rich and O-rich dust depend on the mass of the progenitor. The larger the progenitor, the larger is the O-core, and therefore O-rich dust dominates over other components.

(i) The size distributions of dust grains in $\mathrm{SN}$ ejecta are functions of dust type and epochs of formation. They cannot be generalised as power laws of any exponent, contrary to the general assumption.

(j) A clumpy ejecta of supernova forms larger grains at high densities. These grains are most likely to survive the passage of the reverse shock in the later phase of the evolution.

(k) Theory predicts that a typical type II-P supernova can produce dust masses between 0.02 to $0.2 \mathrm{M}_{\odot}$, while measured dust masses range from $10^{-6}$ to $1 \mathrm{M}_{\odot}$.

(1) A 1-D model can precisely determine the trends and upper or lower limits for the scenario. However, in order to incorporate the impact of mixing and inhomogeneous deposition of radioactive energy in the ejecta, multi-D dynamical modeling of the ejecta is necessary.

Acknowledgements The authors deeply acknowledge the support of the International Space Science Institute in Bern, Switzerland and their successful endeavor to organise the workshop on Supernovae. We also sincerely thank our collaborator, Dr. Isabelle Cherchneff, for the discussions and feedbacks at the workshop and also while writing. A.S. acknowledges the support of NASA Astrophysics Theory grant NNX15AD27G and the CUA grant 361209. Also, E.R.M. wishes to acknowledge the support of Academy of Finland grant 1285769 and M.M. acknowledges support from an STFC Ernest Rutherford fellowship (ST/L003597/1). 
Open Access This article is distributed under the terms of the Creative Commons Attribution 4.0 International License (http://creativecommons.org/licenses/by/4.0/), which permits unrestricted use, distribution, and reproduction in any medium, provided you give appropriate credit to the original author(s) and the source, provide a link to the Creative Commons license, and indicate if changes were made.

\section{References}

F. Abellán, R. Indebetouw, J.M. Marcaide et al., Very deep inside the sn 1987a core ejecta: molecular structures seen in 3d. Astrophys. J. Lett. (2017)

D.K. Aitken, C.H. Smith, S.D. James, P.F. Roche, A.R. Hyland, P.J. McGregor, 10 micron spectral observations of SN1987A - the first year. Mon. Not. R. Astron. Soc. 235, 19P-31P (1988)

S. Amari, L.R. Nittler, E. Zinner, R.S. Lewis, Continued search for rare types of presolar SiC-grains X and Y, in Lunar and Planetary Science Conference, vol. 28 (1997), p. 33

J.E. Andrews, B.E.K. Sugerman, G.C. Clayton, J.S. Gallagher, M.J. Barlow, J. Clem, B. Ercolano, J. Fabbri, M. Meixner, M. Otsuka, D.L. Welch, R. Wesson, Photometric and spectroscopic evolution of the IIP SN 2007it to day 944. Astrophys. J. 731, 47 (2011). 1102.2431. https://doi.org/10.1088/0004-637X/ $731 / 1 / 47$

R.G. Arendt, An infrared survey of Galactic supernova remnants. Astrophys. J. Suppl. Ser. 70, 181-212 (1989)

R.G. Arendt, E. Dwek, S.H. Moseley, Newly synthesized elements and pristine dust in the Cassiopeia A supernova remnant. Astrophys. J. 521(1), 234-245 (1999)

R.G. Arendt, E. Dwek, G. Kober, J. Rho, U. Hwang, Interstellar and ejecta dust in the Cas A supernova remnant. Astrophys. J. 786(1), 55 (2014)

R. Barbon, F. Ciatti, L. Rosino, Photometric properties of type II supernovae. Astron. Astrophys. 72, 287-292 (1979)

R. Barbon, V. Buondí, E. Cappellaro, M. Turatto, The Asiago Supernova Catalogue-10 years after. Astron. Astrophys. Suppl. 139, 531-536 (1999). astro-ph/9908046. https://doi.org/10.1051/aas:1999404

M.J. Barlow, B.E.K. Sugerman, J. Fabbri, M. Meixner, R.S. Fisher, J.E. Bowey, N. Panagia, B. Ercolano, G.C. Clayton, M. Cohen, T.M. Gledhill, K.D. Gordon, A.G.G.M. Tielens, A.A. Zijlstra, Detection of a massive dust shell around the type II supernova SN 2002hh. Astrophys. J. 627(2), L113-L116 (2005)

M.J. Barlow, O. Krause, B.M. Swinyard, B. Sibthorpe, M.A. Besel, R. Wesson, R.J. Ivison, L. Dunne, W.K. Gear, H.L. Gomez, P.C. Hargrave, T. Henning, S.J. Leeks, T.L. Lim, G. Olofsson, E.T. Polehampton, A Herschel PACS and SPIRE study of the dust content of the Cassiopeia A supernova remnant. Astron. Astrophys. 518, L138 (2010). 1005.2688. https://doi.org/10.1051/0004-6361/201014585

M.J. Barlow, B.M. Swinyard, P.J. Owen, J. Cernicharo, H.L. Gomez, R.J. Ivison, O. Krause, T.L. Lim, M. Matsuura, S. Miller, G. Olofsson, E.T. Polehampton, Detection of a noble gas molecular ion, 36ArH+, in the Crab Nebula. Science 342(6164), 1343-1345 (2013)

E.E. Barnard, On the dark markings of the sky, with a catalogue of 182 such objects. Astrophys. J. 49, 1-24 (1919). https://doi.org/10.1086/142439

R.A. Benjamin, E. Churchwell, B.L. Babler, T.M. Bania, D.P. Clemens, M. Cohen, J.M. Dickey, R. Indebetouw, J.M. Jackson, H.A. Kobulnicky, A. Lazarian, A.P. Marston, J.S. Mathis, M.R. Meade, S. Seager, S.R. Stolovy, C. Watson, B.A. Whitney, M.J. Wolff, M.G. Wolfire, GLIMPSE. I. An SIRTF legacy project to map the inner galaxy. Publ. Astron. Soc. Pac. 115, 953 (2003)

T.J. Bernatowicz, E. Zinner (eds.), Astrophysical Implications of the Laboratory Study of Presolar Materials. American Institute of Physics Conference Series, vol. 402 (1997)

F. Bertoldi, C.L. Carilli, P. Cox, X. Fan, M.A. Strauss, A. Beelen, A. Omont, R. Zylka, Dust emission from the most distant quasars. Astron. Astrophys. 406, L55-L58 (2003). astro-ph/0305116. https://doi.org/10.1051/0004-6361:20030710

A. Bevan, M.J. Barlow, Modelling supernova line profile asymmetries to determine ejecta dust masses: SN 1987A from days 714 to 3604. Mon. Not. R. Astron. Soc. 456(2), 1269-1293 (2016)

S. Bianchi, R. Schneider, Dust formation and survival in supernova ejecta. Mon. Not. R. Astron. Soc. 378, 973-982 (2007). 0704.0586. https://doi.org/10.1111/j.1365-2966.2007.11829.x

C. Biscaro, I. Cherchneff, Molecules and dust in Cassiopeia A. I. Synthesis in the supernova phase and processing by the reverse shock in the clumpy remnant. Astron. Astrophys. 564, A25 (2014). 1401.5594. https://doi.org/10.1051/0004-6361/201322932

C. Biscaro, I. Cherchneff, Molecules and dust in Cassiopeia A. II. Dust sputtering and diagnosis of supernova dust survival in remnants. Astron. Astrophys. 589, A132 (2016). 1511.05487. https://doi.org/10.1051/0004-6361/201527769 
S. Blinnikov, P. Lundqvist, O. Bartunov, K. Nomoto, K. Iwamoto, Radiation hydrodynamics of SN 1987A. I. Global analysis of the light curve for the first 4 months. Astrophys. J. 532, 1132-1149 (2000). astro-ph/9911205. https://doi.org/10.1086/308588

J.M. Blondin, K.J. Borkowski, S.P. Reynolds, Dynamics of Fe bubbles in young supernova remnants. Astrophys. J. 557, 782-791 (2001). astro-ph/0010285. https://doi.org/10.1086/321674

P. Bouchet, I.J. Danziger, Infrared photometry and spectrophotometry of supernova 1987A—part two1987NOV to 1991MAR observations. Astron. Astrophys. 273, 451 (1993)

P. Bouchet, I.J. Danziger, L.B. Lucy, Bolometric light curve of SN 1987A-results from day 616 to 1316 after outburst. Astron. J. 102, 1135-1146 (1991)

P. Bouchet, J.M. De Buizer, N.B. Suntzeff, I.J. Danziger, T.L. Hayward, C.M. Telesco, C. Packham, Highresolution mid-infrared imaging of SN 1987A. Astrophys. J. 611(1), 394-398 (2004)

P. Bouchet, E. Dwek, J. Danziger, R.G. Arendt, I.J.M. De Buizer, S. Park, N.B. Suntzeff, R.P. Kirshner, P. Challis, SN 1987A after 18 years: mid-infrared Gemini and Spitzer observations of the remnant. Astrophys. J. 650, 212 (2006)

D. Branch, M. Livio, L.R. Yungelson, F.R. Boffi, E. Baron, In search of the progenitors of type IA supernovae. Publ. Astron. Soc. Pac. 107, 1019 (1995). https://doi.org/10.1086/133657

D.E. Brownlee, Cosmic dust-collection and research. Annu. Rev. Earth Planet. Sci. 13, 147-173 (1985). https://doi.org/10.1146/annurev.ea.13.050185.001051

F. Calura, R. Gilli, C. Vignali, F. Pozzi, A. Pipino, F. Matteucci, The dust content of QSO hosts at high redshift. Mon. Not. R. Astron. Soc. 438, 2765-2783 (2014). 1312.1087. https://doi.org/10.1093/ mnras/stt2329

R.M. Catchpole, P.A. Whitelock, M.W. Feast, J.M. Menzies, I.S. Glass, F. Marang, J.D. Laing, J.H. Spencer Jones, G. Roberts, L.A. Balona, B.S. Carter, C.D. Laney, L.T. Evans, K. Sekiguchi, G.G. Hutchinson, R. Maddison, J. Albinson, A. Evans, F.A. Allen, H. Winkler, A. Fairall, C. Corbally, J.K. Davies, Q.A. Parker, Spectroscopic and photometric observations of SN 1987A. III-days 135 to 260. Mon. Not. R. Astron. Soc. 231, 75P (1988)

I. Cherchneff, Dust formation in massive stars and their explosive ends, in Hot and Cool: Bridging Gaps in Massive Star Evolution, ed. by C. Leitherer, P.D. Bennett, P.W. Morris, J.T. Van Loon. Astronomical Society of the Pacific Conference Series, vol. 425 (2010), p. 237. 0909.0164

I. Cherchneff, The inner wind of IRC+10216 revisited: new exotic chemistry and diagnostic for dust condensation in carbon stars. Astron. Astrophys. 545, A12 (2012). 1111.6809. https://doi.org/10.1051/ 0004-6361/201118542

I. Cherchneff, The chemistry of dust formation in red supergiants, in Betelgeuse Workshop 2012, ed. by P. Kervella, T. Le Bertre, G. Perrin. EAS Publications Series, vol. 60 (2013), pp. 175-184. 1303.2805. https://doi.org/10.1051/eas/1360020

I. Cherchneff, E. Dwek, The chemistry of population III supernova ejecta. I. Formation of molecules in the early universe. Astrophys. J. 703, 642-661 (2009). 0907.3621. https://doi.org/10.1088/0004-637X/ $703 / 1 / 642$

I. Cherchneff, E. Dwek, The chemistry of population III supernova ejecta. II. The nucleation of molecular clusters as a diagnostic for dust in the early universe. Astrophys. J. 713, 1-24 (2010). 1002.3060. https://doi.org/10.1088/0004-637X/713/1/1

I. Cherchneff, S. Lilly, Primordial massive supernovae as the first molecular factories in the early universe. Astrophys. J. Lett. 683, L123 (2008). 0807.2511. https://doi.org/10.1086/591906

I. Cherchneff, A. Sarangi, Molecules in supernova ejecta, in IAU Symposium, vol. 280, ed. by J. Cernicharo, R. Bachiller (2011), pp. 228-236. 1107.5668. https://doi.org/10.1017/S1743921311025002

R.A. Chevalier, The radio and X-ray emission from type II supernovae. Astrophys. J. 259, 302-310 (1982). https://doi.org/10.1086/160167

H. Chihara, C. Koike, A. Tsuchiyama, S. Tachibana, D. Sakamoto, Compositional dependence of infrared absorption spectra of crystalline silicates. I. Mg-Fe pyroxenes. Astron. Astrophys. 391, 267-273 (2002). https://doi.org/10.1051/0004-6361:20020791

B.G. Choi, G.R. Huss, G.J. Wasserburg, Oxygen, magnesium, calcium, and titanium isotopes in asymptotic giant branch and supernova oxides. Meteorit. Planet. Sci. Suppl. 33, A32 (1998)

D.D. Clayton, Sudden grain nucleation and growth in supernova and nova ejecta. Astrophys. Space Sci. 65, 179-189 (1979). https://doi.org/10.1007/BF00643499

D.D. Clayton, L.R. Nittler, Astrophysics with presolar stardust. Annu. Rev. Astron. Astrophys. 42, 39-78 (2004). https://doi.org/10.1146/annurev.astro.42.053102.134022

D.D. Clayton, D. Arnett, J. Kane, B.S. Meyer, Type X silicon carbide presolar grains: type Ia supernova condensates? Astrophys. J. 486, 824-834 (1997). https://doi.org/10.1086/304545

D.D. Clayton, W. Liu, A. Dalgarno, Condensation of carbon in radioactive supernova gas. Science 283, 1290 (1999). https://doi.org/10.1126/science.283.5406.1290 
L. Colangeli, V. Mennella, P. Palumbo, A. Rotundi, E. Bussoletti, Mass extinction coefficients of various submicron amorphous carbon grains: tabulated values from $40 \mathrm{NM}$ to $2 \mathrm{~mm}$. Astron. Astrophys. Suppl. 113, $561(1995)$

I.J. Danziger, P. Bouchet, R.A.E. Fosbury, C. Gouiffes, L.B. Lucy, SN 1987A—observational results obtained at ESO, in Supernova 1987A in the Large Magellanic Cloud, ed. by M. Kafatos, A.G. Michalitsianos (1988), pp. 37-50

I. Danziger, C. Gouiffes, P. Bouchet, L.B. Lucy, Supernova 1987a in the large magellanic cloud, in $I A U$ Circular, vol. 4747 (1989)

I.J. Danziger, L.B. Lucy, P. Bouchet, C. Gouiffes, Molecules dust and ionic abundances in supernova 1987A, in Supernovae, ed. by S.E. Woosley (1991), p. 69

I. De Looze, M.J. Barlow, B.M. Swinyard, J. Rho, H.L. Gomez, M. Matsuura, R. Wesson, The dust mass in Cassiopeia A from a spatially resolved Herschel analysis. Mon. Not. R. Astron. Soc. 465(3), 3309-3342 (2017)

E. Di Carlo, C. Corsi, A.A. Arkharov, F. Massi, V.M. Larionov, N.V. Efimova, M. Dolci, N. Napoleone, A. Di Paola, Near-infrared observations of the type Ib supernova SN 2006jc: evidence of interactions with dust. Astrophys. J. 684, 471-480 (2008). 0712.3855. https://doi.org/10.1086/590051

B. Donn, J.A. Nuth, Does nucleation theory apply to the formation of refractory circumstellar grains? Astrophys. J. 288, 187-190 (1985). https://doi.org/10.1086/162779

J. Dorschner, B. Begemann, T. Henning, C. Jaeger, H. Mutschke, Steps toward interstellar silicate mineralogy. II. Study of Mg-Fe-silicate glasses of variable composition. Astron. Astrophys. 300, 503 (1995)

T. Dotani, K. Hayashida, H. Inoue, M. Itoh, K. Koyama, F. Makino, K. Mitsuda, T. Murakami, M. Oda, Y. Ogawara, S. Takano, Y. Tanaka, A. Yoshida, K. Makishima, T. Ohashi, N. Kawai, M. Matsuoka, R. Hoshi, S. Hayakawa, T. Kii, H. Kunieda, F. Nagase, Y. Tawara, I. Hatsukade, S. Kitamoto, S. Miyamoto, H. Tsunemi, K. Yamashita, M. Nakagawa, M. Yamauchi, M.J.L. Turner, K.A. Pounds, H.D. Thomas, G.C. Stewart, A.M. Cruise, B.E. Patchett, D.H. Reading, Discovery of an unusual hard X-ray source in the region of supernova 1987A. Nature 330, 230 (1987). https://doi.org/10.1038/330230a0

T. Douvion, P.O. Lagage, E. Pantin, Cassiopeia A dust composition and heating. Astron. Astrophys. 369, 589-593 (2001). https://doi.org/10.1051/0004-6361:20010053

B.T. Draine, The infrared signature of graphite grains. Astrophys. J. Lett. 277, L71-L74 (1984). https:// doi.org/10.1086/184206

B.T. Draine, Interstellar dust grains. Annu. Rev. Astron. Astrophys. 41, 241-289 (2003). astro-ph/0304489. https://doi.org/10.1146/annurev.astro.41.011802.094840

M.R. Drout, D. Milisavljevic, J. Parrent, R. Margutti, A. Kamble, A.M. Soderberg, P. Challis, R. Chornock, W. Fong, S. Frank, N. Gehrels, M.L. Graham, E. Hsiao, K. Itagaki, M. Kasliwal, R.P. Kirshner, D. Macomb, G.H. Marion, J. Norris, M.M. Phillips, The double-peaked sn 2013ge: a type Ib/c SN with an asymmetric mass ejection or an extended progenitor envelope. Astrophys. J. 821(1), 57 (2016)

P. Dunk, N. Kaiser, M. Mullet-Gas et al., The smallest stable fullerene, $M @ C_{28}(M=T i, Z r$, U): stabilization and growth from carbon vapor. J. Am. Chem. Soc. 134, 9380 (2012). https://doi.org/10.1021/ja302398h

L. Dunne, S. Eales, R. Ivison, H. Morgan, M. Edmunds, Type II supernovae as a significant source of interstellar dust. Nature 424, 285-287 (2003). astro-ph/0307320. https://doi.org/10.1038/nature01792

E. Dwek, Will dust black out SN 1987A? Astrophys. J. 329, 814 (1988)

E. Dwek, The evolution of the elemental abundances in the gas and dust phases of the galaxy. Astrophys. J. 501, 643 (1998). astro-ph/9707024. https://doi.org/10.1086/305829

E. Dwek, The supernova origin of interstellar dust. Science 313(5784), 178-180 (2006). http://science. sciencemag.org/content/313/5784/178.full.pdf. https://doi.org/10.1126/science.1130423

E. Dwek, R.G. Arendt, Infrared echoes reveal the shock breakout of the Cas A supernova. Astrophys. J. 685, 976-987 (2008). 0802.0221. https://doi.org/10.1086/589988

E. Dwek, R.G. Arendt, The evolution of dust mass in the ejecta of SN1987A. Astrophys. J. 810(1), 75 (2015)

E. Dwek, I. Cherchneff, The origin of dust in the early universe: probing the star formation history of galaxies by their dust content. Astrophys. J. 727, 63 (2011). 1011.1303. https://doi.org/10.1088/0004-637X/ $727 / 2 / 63$

E. Dwek, M.G. Hauser, H.L. Dinerstein, F.C. Gillett, W.L. Rice, Physical processes and infrared emission from the Cassiopeia A supernova remnant. Astrophys. J. 315, 571-579 (1987)

E. Dwek, S.H. Moseley, W. Glaccum, J.R. Graham, R.F. Loewenstein, R.F. Silverberg, R.K. Smith, Dust and gas contributions to the energy output of SN 1987A on day 1153. Astrophys. J. 389, L21-L24 (1992)

E. Dwek, F. Galliano, A.P. Jones, The evolution of dust in the early universe with applications to the galaxy SDSS J1148+5251. Astrophys. J. 662, 927-939 (2007). 0705.3799. https://doi.org/10.1086/518430

E. Dwek, R.G. Arendt, P. Bouchet, D.N. Burrows, P. Challis, I.J. Danziger, J.M. De Buizer, R.D. Gehrz, R.P. Kirshner, R.A. McCray, S. Park, E.F. Polomski, C.E. Woodward, Infrared and X-ray evidence for circumstellar grain destruction by the blast wave of supernova 1987A. Astrophys. J. 676, 1029 (2008) 
A. Elmhamdi, N.N. Chugai, I.J. Danziger, Light curves and $\mathrm{H} \alpha$ luminosities as indicators of ${ }^{56} \mathrm{Ni}$ mass in type IIP supernovae. Astron. Astrophys. 404, 1077-1086 (2003a). astro-ph/0304144. https://doi.org/10.1051/0004-6361:20030522

A. Elmhamdi, I.J. Danziger, N. Chugai, A. Pastorello, M. Turatto, E. Cappellaro, G. Altavilla, S. Benetti, F. Patat, M. Salvo, Photometry and spectroscopy of the type IIP SN 1999em from outburst to dust formation. Mon. Not. R. Astron. Soc. 338, 939-956 (2003b). astro-ph/0209623. https://doi.org/ 10.1046/j.1365-8711.2003.06150.x

A. Elmhamdi, I.J. Danziger, E. Cappellaro, M. Della Valle, C. Gouiffes, M.M. Phillips, M. Turatto, SN Ib 1990I: clumping and dust in the ejecta? Astron. Astrophys. 426, 963-977 (2004). astro-ph/0407145. https://doi.org/10.1051/0004-6361:20041318

L. Ensman, A. Burrows, Shock breakout in SN 1987A. Astrophys. J. 393, 742-755 (1992). https://doi.org/ $10.1086 / 171542$

B. Ercolano, M.J. Barlow, B.E.K. Sugerman, Dust yields in clumpy supernova shells: SN 1987A revisited. Mon. Not. R. Astron. Soc. 375, 753-763 (2007). astro-ph/0611719. https://doi.org/10.1111/ j.1365-2966.2006.11336.x

J. Fabbri, M. Otsuka, M.J. Barlow, J.S. Gallagher, R. Wesson, B.E.K. Sugerman, G.C. Clayton, M. Meixner, J.E.E. Andrews, D.L. Welch, B. Ercolano, The effects of dust on the optical and infrared evolution of SN 2004et. Mon. Not. R. Astron. Soc. 418(2), 1285-1307 (2011)

D. Fabian, C. Jäger, T. Henning, J. Dorschner, H. Mutschke, Steps toward interstellar silicate mineralogy. V. Thermal evolution of amorphous magnesium silicates and silica. Astron. Astrophys. 364, 282-292 (2000)

D. Fabian, T. Posch, H. Mutschke, F. Kerschbaum, J. Dorschner, Infrared optical properties of spinels. A study of the carrier of the 13, 17 and $32 \mu \mathrm{m}$ emission features observed in ISO-SWS spectra of oxygen-rich AGB stars. Astron. Astrophys. 373, 1125-1138 (2001). https://doi.org/10.1051/0004-6361:20010657

A. Fassia, W.P.S. Meikle, N. Chugai, T.R. Geballe, P. Lundqvist, N.A. Walton, D. Pollacco, S. Veilleux, G.S. Wright, M. Pettini, T. Kerr, E. Puchnarewicz, P. Puxley, M. Irwin, C. Packham, S.J. Smartt, D. Harmer, Optical and infrared spectroscopy of the type IIn SN 1998S: days 3-127. Mon. Not. R. Astron. Soc. 325, 907-930 (2001). astro-ph/0011340. https://doi.org/10.1046/j.1365-8711.2001.04282.x

J. Feder, K. Russell, J. Lothe, G. Pound, Homogeneous nucleation and growth of droplets in vapours. Adv. Phys. 15(57), 111-178 (1966). https://doi.org/10.1080/00018736600101264

G.J. Ferland, N. Abel, K. Davidson, N. Smith, Physical conditions in the homunculus, in The Fate of the Most Massive Stars, ed. by R. Humphreys, K. Stanek. Astronomical Society of the Pacific Conference Series, vol. 332 (2005), p. 294. astro-ph/0503633

R. Fesen, W.P. Blair, Optical identification of dust within the Crab Nebula's filaments. Astrophys. J. 351 , L45 (1990)

R.A. Fesen, M.C. Hammell, J. Morse, R.A. Chevalier, K.J. Borkowski, M.A. Dopita, C.L. Gerardy, S.S. Lawrence, J.C. Raymond, S. van den Bergh, The expansion asymmetry and age of the Cassiopeia A supernova remnant. Astrophys. J. 645(1), 283-292 (2006)

A.V. Filippenko, Optical spectra of supernovae. Annu. Rev. Astron. Astrophys. 35, 309-355 (1997). https:// doi.org/10.1146/annurev.astro.35.1.309

C. Fransson, R.A. Chevalier, Late emission from supernovae-a window on stellar nucleosynthesis. Astrophys. J. 343, 323-342 (1989). https://doi.org/10.1086/167707

C. Fransson, J. Larsson, K. Migotto, D. Pesce, P. Challis, R.A. Chevalier, K. France, R.P. Kirshner, B. Leibundgut, P. Lundqvist, R.A. McCray, J. Spyromilio, F. Taddia, A. Jerkstrand, S. Mattila, N. Smith, J. Sollerman, J.C. Wheeler, A. Crotts, P. Garnavich, K. Heng, S.S. Lawrence, N. Panagia, C.S.J. Pun, G. Sonneborn, B.E.K. Sugerman, The destruction of the circumstellar ring of SN 1987A. Astrophys. J. 806(1), L19 (2015)

C. Fransson, J. Larsson, J. Spyromilio, B. Leibundgut, R.A. McCray, A. Jerkstrand, Discovery of molecular hydrogen in SN 1987A. Astrophys. J. 821(1), L5 (2016)

C.L. Fryer, S.E. Woosley, A. Heger, Pair-instability supernovae, gravity waves, and gamma-ray transients. Astrophys. J. 550, 372-382 (2001). astro-ph/0007176. https://doi.org/10.1086/319719

C. Gall, J. Hjorth, A.C. Andersen, Production of dust by massive stars at high redshift. Astron. Astrophys. Rev. 19, 43 (2011). 1108.0403. https://doi.org/10.1007/s00159-011-0043-7

C. Gall, J. Hjorth, D. Watson, E. Dwek, J.R. Maund, O.D. Fox, G. Leloudas, D. Malesani, A.C. Day-Jones, Rapid formation of large dust grains in the luminous supernova 2010jl. Nature 511(7509), 326-329 (2014)

J.S. Gallagher, B.E.K. Sugerman, G.C. Clayton, J.E. Andrews, J. Clem, M.J. Barlow, B. Ercolano, J. Fabbri, M. Otsuka, R. Wesson, M. Meixner, Optical and infrared analysis of type II SN 2006bc. Astrophys. J. 753, 109 (2012). 1205.5517. https://doi.org/10.1088/0004-637X/753/2/109

C.L. Gerardy, R.A. Fesen, P. Höflich, J.C. Wheeler, Detection of CO and dust emission in near-infrared spectra of SN 1998S. Astron. J. 119(6), 2968-2981 (2000) 
C.L. Gerardy, R.A. Fesen, K. Nomoto, K. Maeda, P. Höflich, J.C. Wheeler, Carbon monoxide in the type Ic supernova 2000ew. Publ. Astron. Soc. Jpn. 54(6), 905-910 (2002)

D. Gobrecht, I. Cherchneff, A. Sarangi, J.M.C. Plane, S.T. Bromley, Dust formation in the oxygenrich AGB star IK Tauri. Astron. Astrophys. 585, A6 (2016). 1509.07613. https://doi.org/10.1051/ 0004-6361/201425363

H.L. Gomez, O. Krause, M.J. Barlow, B.M. Swinyard, P.J. Owen, C.J.R. Clark, M. Matsuura, E.L. Gomez, J. Rho, M.A. Besel, J. Bouwman, W.K. Gear, T. Henning, R.J. Ivison, E.T. Polehampton, B. Sibthorpe, A cool dust factory in the Crab Nebula: a Herschel study of the filaments. Astrophys. J. 760, 96 (2012). 1209.5677. https://doi.org/10.1088/0004-637X/760/1/96

E.V. Gotthelf, B. Koralesky, L. Rudnick, T.W. Jones, U. Hwang, R. Petre, Chandra detection of the forward and reverse shocks in Cassiopeia A. Astrophys. J. 552(1), L39-L43 (2001)

T.P.M. Goumans, S.T. Bromley, Efficient nucleation of stardust silicates via heteromolecular homogeneous condensation. Mon. Not. R. Astron. Soc. 420, 3344-3349 (2012). https://doi.org/10.1111/j.13652966.2011.20255.x

J.R. Graham, G.S. Wright, A.J. Longmore, Infrared spectroscopy and imaging of the Crab Nebula. Astrophys. J. 352, 172-183 (1990)

D.A. Green, Galactic supernova remnants: an updated catalogue and some statistics. Bull. Astron. Soc. India 32, 335-370 (2004)

D.A. Green, R.J. Tuffs, C.C. Popescu, Far-infrared and submillimetre observations of the Crab Nebula. Mon. Not. R. Astron. Soc. 355(4), 1315-1326 (2004)

T. Grenman, G.F. Gahm, E. Elfgren, Dusty globules in the Crab Nebula. Astron. Astrophys. 599, A110 (2017). 1610.08449. https://doi.org/10.1051/0004-6361/201629693

E. Grün, B.A.S. Gustafson, S. Dermott, H. Fechtig, in Interplanetary Dust, ed. by E. Grün, B.A.S. Gustafson, S. Dermott, H. Fechtig Astronomy and Astrophysics Library (Springer, Berlin, 2001), 804 p. ISBN 3-540-42067-3

M.R. Haas, E.F. Erickson, S.D. Lord, D.J. Hollenbach, S.W.J. Colgan, M.G. Burton, Velocity-resolved farinfrared spectra of forbidden Fe II-evidence for mixing and clumping in SN 1987A. Astrophys. J. 360, 257-266 (1990). https://doi.org/10.1086/169115

N.J. Hammer, H.T. Janka, E. Müller, Three-dimensional simulations of mixing instabilities in supernova explosions. Astrophys. J. 714, 1371-1385 (2010a). 0908.3474. https://doi.org/10.1088/0004-637X/ $714 / 2 / 1371$

N.J. Hammer, H.T.T. Janka, E. Muller, Three-dimensional simulations of mixing instabilities in supernova explosions. Astrophys. J. 714(2), 1371-1385 (2010b)

M. Hamuy, Observed and physical properties of core-collapse supernovae. Astrophys. J. 582, 905-914 (2003). astro-ph/0209174. https://doi.org/10.1086/344689

R.W. Hanuschik, G. Thimm, J. Dachs, H-alpha fine-structure in SN 1987A within the first 111 days. Mon. Not. R. Astron. Soc. 234, 41P-49P (1988). https://doi.org/10.1093/mnras/234.1.41P

T.J. Harries, J.D. Monnier, N.H. Symington, R. Kurosawa, Three-dimensional dust radiative-transfer models: the Pinwheel Nebula of WR 104. Mon. Not. R. Astron. Soc. 350, 565-574 (2004). astro-ph/0401574. https://doi.org/10.1111/j.1365-2966.2004.07668.x

P. Harvey, D. Lester, H. Dinerstein, B. Smith, C. Colome, Far-IR monitoring of SN1987A. Bull. Am. Astron. Soc. 21, 1215 (1989)

A. Heger, S.E. Woosley, The nucleosynthetic signature of population III. Astrophys. J. 567, 532-543 (2002). astro-ph/0107037. https://doi.org/10.1086/338487

T. Henning, B. Begemann, H. Mutschke, J. Dorschner, Optical properties of oxide dust grains. Astron. Astrophys. Suppl. 112, 143 (1995)

M. Herant, S.E. Woosley, Postexplosion hydrodynamics of supernovae in red supergiants. Astrophys. J. 425, 814-828 (1994). https://doi.org/10.1086/174026

J.J. Hester, The Crab Nebula: an astrophysical chimera. Annu. Rev. Astron. Astrophys. 46(1), 127-155 (2008)

D.J. Hillier, K. Davidson, K. Ishibashi, T. Gull, On the nature of the central source in $\eta$ Carinae. Astrophys. J. 553, 837-860 (2001). https://doi.org/10.1086/320948

D.C. Hines, G.H. Rieke, K.D. Gordon, J. Rho, K.A. Misselt, C.E. Woodward, M.W. Werner, O. Krause, W.B. Latter, C.W. Engelbracht, E. Egami, D.M. Kelly, J. Muzerolle, J.A. Stansberry, K.Y.L. Su, J.E. Morrison, E.T. Young, A. Noriega-Crespo, D.L. Padgett, R.D. Gehrz, E. Polomski, J.W. Beeman, E.E. Haller, Imaging of the supernova remnant Cassiopeia A with the multiband imaging photometer for Spitzer (MIPS). Astrophys. J. Suppl. Ser. 154(1), 290-295 (2004)

D.C. Hines, O. Krause, G.H. Rieke, X. Fan, M. Blaylock, G. Neugebauer, Spitzer observations of highredshift QSOs. Astrophys. J. Lett. 641, L85-L88 (2006). astro-ph/0604347. https://doi.org/10.1086/ 504109 
A.M. Hofmeister, E. Keppel, A.K. Speck, Absorption and reflection infrared spectra of $\mathrm{MgO}$ and other diatomic compounds. Mon. Not. R. Astron. Soc. 345, 16-38 (2003). https://doi.org/10.1046/ j.1365-8711.2003.06899.x

P. Hoppe, Measurements of presolar grains, in Nuclei in the Cosmos (2010)

P. Hoppe, U. Ott, Mainstream silicon carbide grains from meteorites, in American Institute of Physics Conference Series, vol. 402 ed. by T.J. Bernatowicz, E. Zinner. (1997), pp. 27-58. https://doi.org/ $10.1063 / 1.53314$

F. Hoyle, N.C. Wickramasinghe, Dust in supernova explosions. Nature 226(5240), 62-63 (1970)

J.P. Hughes, C.E. Rakowski, D.N. Burrows, P.O. Slane, Nucleosynthesis and mixing in Cassiopeia A. Astrophys. J. Lett. 528, L109-L113 (2000). astro-ph/9910474. https://doi.org/10.1086/312438

D.J. Hunter, S. Valenti, R. Kotak, W.P.S. Meikle, S. Taubenberger, A. Pastorello, S. Benetti, V. Stanishev, S.J. Smartt, C. Trundle, A.A. Arkharov, F. Bufano, E. Cappellaro, E. Di Carlo, M. Dolci, N. EliasRosa, S. Frandsen, J.U. Fynbo, U. Hopp, V.M. Larionov, P. Laursen, P. Mazzali, H. Navasardyan, C. Ries, A. Riffeser, L. Rizzi, D.Y. Tsvetkov, M. Turatto, S. Wilke, Extensive optical and near-infrared observations of the nearby, narrow-lined type Ic SN 2007gr: days 5 to 415. Astron. Astrophys. 508(1), 371-389 (2009)

K.M. Hynes, F. Gyngard, in Lunar and Planetary Science Conference, vol. 40 (2009), p. 1198. The presolar grain database: http://presolar.wustl.edu/ pgd,

R. Indebetouw, M. Matsuura, E. Dwek, G. Zanardo, M.J. Barlow, M. Baes, P. Bouchet, D.N. Burrows, R.A. Chevalier, G.C. Clayton, C. Fransson, R.P. Kirshner, M. Lakicevic, K.S. Long, P. Lundqvist, I. MartíVidal, J. Marcaide, R.A. McCray, M. Meixner, C.Y. Ng, S. Park, G. Sonneborn, L. Staveley-Smith, C. Vlahakis, Dust production and particle acceleration in supernova 1987a revealed with ALMA. Astrophys. J. 782(1), L2 (2014)

C. Inserra, M. Turatto, A. Pastorello, S. Benetti, E. Cappellaro, M.L. Pumo, L. Zampieri, I. Agnoletto, F. Bufano, M.T. Botticella, M. Della Valle, N. Elias Rosa, T. Iijima, S. Spiro, S. Valenti, The type IIP SN 2007od in UGC 12846: from a bright maximum to dust formation in the nebular phase. Mon. Not. R. Astron. Soc. 417, 261-279 (2011). 1102.5468. https://doi.org/10.1111/j.1365-2966.2011.19128.x

M.Z. Jacobson, Fundamentals of Atmospheric Modeling, 2nd edn. (Cambridge University Press, Cambridge, 2005)

A. Jerkstrand, C. Fransson, C. Kozma, The ${ }^{44}$ Ti-powered spectrum of SN 1987A. Astron. Astrophys. 530, A45 (2011). 1103.3653. https://doi.org/10.1051/0004-6361/201015937

C.C. Joggerst, A. Almgren, S.E. Woosley, Three-dimensional simulations of Rayleigh-Taylor mixing in core-collapse supernovae with Castro. Astrophys. J. 723, 353-363 (2010). 1009.3336. https://doi.org/ $10.1088 / 0004-637 \mathrm{X} / 723 / 1 / 353$

A.P. Jones, Dust destruction processes, in Astrophysics of Dust, ed. by A.N. Witt, G.C. Clayton, B.T. Draine. Astronomical Society of the Pacific Conference Series, vol. 309 (2004), p. 347

A.P. Jones, A.G.G.M. Tielens, D.J. Hollenbach, C.F. McKee, Grain destruction in shocks in the interstellar medium. Astrophys. J. 433, 797 (1994)

J. Kamenetzky, R. McCray, R. Indebetouw, M.J. Barlow, M. Matsuura, M. Baes, J.A.D.L. Blommaert, A. Bolatto, L. Decin, L. Dunne, C. Fransson, J. Glenn, H.L. Gomez, M.A.T. Groenewegen, R. Hopwood, R.P. Kirshner, M. Lakicevic, J. Marcaide, I. Marti-Vidal, M. Meixner, P. Royer, A. Soderberg, G. Sonneborn, L. Staveley-Smith, B.M. Swinyard, G. Van de Steene, P.A.M. van Hoof, J.T. van Loon, J. Yates, G. Zanardo, Carbon monoxide in the cold debris of supernova 1987A. Astrophys. J. Lett. 773, L34 (2013). 1307.6561. https://doi.org/10.1088/2041-8205/773/2/L34

E. Kankare, M. Fraser, S. Ryder, C. Romero-Cañizales, S. Mattila, R. Kotak, P. Laursen, L.A.G. Monard, M. Salvo, P. Väisänen, SN 2005at-a neglected type Ic supernova at 10 Mpc. Astron. Astrophys. 572, A75 (2014). 1409.1758. https://doi.org/10.1051/0004-6361/201424563

F. Kemper, W.J. Vriend, A.G.G.M. Tielens, Erratum: "The absence of crystalline silicates in the diffuse interstellar medium" (ApJ, 609, 826 [2004]). Astrophys. J. 633, 534 (2005). https://doi.org/10.1086/447764

K. Kifonidis, T. Plewa, H.T. Janka, E. Müller, Non-spherical core collapse supernovae. I. Neutrino-driven convection, Rayleigh-Taylor instabilities, and the formation and propagation of metal clumps. Astron. Astrophys. 408, 621-649 (2003). astro-ph/0302239. https://doi.org/10.1051/0004-6361:20030863

K. Kifonidis, T. Plewa, L. Scheck, H.T. Janka, E. Müller, Non-spherical core collapse supernovae. II. The late-time evolution of globally anisotropic neutrino-driven explosions and their implications for SN 1987 A. Astron. Astrophys. 453, 661-678 (2006). astro-ph/0511369. https://doi.org/10.1051/ 0004-6361:20054512

C. Koike, H. Hasegawa, N. Asada, T. Hattori, The extinction coefficients in mid- and far-infrared of silicate and iron-oxide minerals of interest for astronomical observations. Astrophys. Space Sci. 79, 77-85 (1981). https://doi.org/10.1007/BF00655906

C. Koike, C. Kaito, T. Yamamoto, H. Shibai, S. Kimura, H. Suto, Extinction spectra of corundum in the wavelengths from UV to FIR. Icarus 114, 203-214 (1995). https://doi.org/10.1006/icar.1995.1055 
C. Koike, H. Chihara, A. Tsuchiyama, H. Suto, H. Sogawa, H. Okuda, Compositional dependence of infrared absorption spectra of crystalline silicate. II. Natural and synthetic olivines. Astron. Astrophys. 399, 1101-1107 (2003). https://doi.org/10.1051/0004-6361:20021831

R. Kotak, P. Meikle, S.D. van Dyk, P.A. Höflich, S. Mattila, Early-time Spitzer observations of the type II Plateau supernova SN 2004dj. Astrophys. J. Lett. 628, L123-L126 (2005). astro-ph/0506407. https://doi.org/10.1086/432719

R. Kotak, W.P.S. Meikle, M. Pozzo, S.D. van Dyk, D. Farrah, R. Fesen, A.V. Filippenko, R.J. Foley, C. Fransson, P.A. Höflich, P. Lundqvist, S. Mattila, J. Sollerman, J.C. Wheeler, Spitzer measurements of atomic and molecular abundances in the type IIP SN 2005af. Astrophys. J. 651(2), L117-L120 (2006)

R. Kotak, W.P.S. Meikle, D. Farrah, C.L. Gerardy, R.J. Foley, S.D. Van Dyk, C. Fransson, P. Lundqvist, J. Sollerman, R. Fesen, A.V. Filippenko, S. Mattila, J.M. Silverman, A.C. Andersen, P.A. Höflich, M. Pozzo, J.C. Wheeler, Dust and the type II-Plateau supernova 2004et. Astrophys. J. 704, 306-323 (2009). 0904.3737. https://doi.org/10.1088/0004-637X/704/1/306

T. Kozasa, H. Hasegawa, K. Nomoto, Formation of dust grains in the ejecta of SN 1987A. Astrophys. J. 344, 325-331 (1989). https://doi.org/10.1086/167801

T. Kozasa, T. Nozawa, N. Tominaga, H. Umeda, K. Maeda, K. Nomoto, Dust in supernovae: formation and evolution, in Cosmic Dust-Near and Far, ed. by T. Henning, E. Grün, J. Steinacker. Astronomical Society of the Pacific Conference Series, vol. 414 (2009), p. 43. 0903.0217

O. Krause, S.M. Birkmann, T. Usuda, T. Hattori, M. Goto, G.H. Rieke, K.A. Misselt, The Cassiopeia A supernova was of type IIb. Science 320(5880), 1195-1197 (2008)

W. Kunkel, B. Madore, I. Shelton, O. Duhalde, F.M. Bateson, A. Jones, B. Moreno, S. Walker, G. Garradd, B. Warner, J. Menzies, Supernova 1987A in the large magellanic cloud. IAU Circ 4316, 1 (1987)

P.O. Lagage, A. Claret, J. Ballet, F. Boulanger, C.J. Cesarsky, D. Cesarsky, C. Fransson, A. Pollock, Dust formation in the Cassiopeia A supernova. Astron. Astrophys. 315, L273-L276 (1996)

Lakicevic et al., Astrophys. J. 799, 50 (2015)

J. Larsson, C. Fransson, J. Spyromilio, B. Leibundgut, P. Challis, R.A. Chevalier, K. France, A. Jerkstrand, R.P. Kirshner, P. Lundqvist, M. Matsuura, R.A. McCray, N. Smith, J. Sollerman, P. Garnavich, K. Heng, S. Lawrence, S. Mattila, K. Migotto, G. Sonneborn, F. Taddia, J.C. Wheeler, Three-dimensional distribution of ejecta in supernova 1987A at 10,000 days. Astrophys. J. 833(2), 147 (2016)

J. Leitner, P. Hoppe, J. Zipfel, Distribution and abundance of presolar silicate and oxide stardust in CR chondrites, in Lunar and Planetary Science Conference, vol. 46 (2015), p. 1874

S. Lepp, A. Dalgarno, R. McCray, Molecules in the ejecta of SN 1987A. Astrophys. J. 358, 262-265 (1990). https://doi.org/10.1086/168981

A. Li, The warm, cold and very cold dusty universe. ArXiv Astrophysics e-prints astro-ph/0503571 (2005)

A. Li, B.T. Draine, On ultrasmall silicate grains in the diffuse interstellar medium. Astrophys. J. Lett. 550, L213-L217 (2001). astro-ph/0012147. https://doi.org/10.1086/319640

M.P. Li, G. Zhao, A. Li, On the crystallinity of silicate dust in the interstellar medium. Mon. Not. R. Astron. Soc. 382, L26-L29 (2007). 0808.4129. https://doi.org/10.1111/j.1745-3933.2007.00382.x

M. Limongi, O. Straniero, A. Chieffi, Massive stars in the range 13-25 $\mathrm{M}_{\text {solar }}$ : evolution and nucleosynthesis. II. The solar metallicity models. Astrophys. J. Suppl. Ser. 129, 625-664 (2000). astro-ph/0003401. https://doi.org/10.1086/313424

W. Liu, A. Dalgarno, Silicon monoxide in SN 1987A. Astrophys. J. 428, 769-776 (1994). https://doi.org/ $10.1086 / 174285$

W. Liu, A. Dalgarno, The oxygen temperature of SN 1987A. Astrophys. J. 454, 472 (1995). https://doi.org/ $10.1086 / 176498$

W. Liu, A. Dalgarno, S. Lepp, Carbon monoxide in SN 1987A. Astrophys. J. 396, 679-685 (1992). https:// doi.org/10.1086/171749

E.D. Loh, J.A. Baldwin, Z.K. Curtis, G.J. Ferland, C.R. O’Dell, A.C. Fabian, P. Salomé, A survey of molecular hydrogen in the Crab Nebula. Astrophys. J. Suppl. Ser. 194(2), 30 (2011)

E.D. Loh, J.A. Baldwin, G.J. Ferland, Z.K. Curtis, C.T. Richardson, A.C. Fabian, P. Salomé, H2 temperatures in the Crab Nebula. Mon. Not. R. Astron. Soc. (2012)

L.B. Lucy, I.J. Danziger, C. Gouiffes, P. Bouchet, Dust condensation in the ejecta of SN 1987 A, in IAU Colloq. 120: Structure and Dynamics of the Interstellar Medium, ed. by G. Tenorio-Tagle, M. Moles, J. Melnick. Lecture Notes in Physics, vol. 350 (Springer, Berlin, 1989), p. 164. https://doi.org/ 10.1007/BFb0114861

R. Maiolino, R. Schneider, E. Oliva, S. Bianchi, A. Ferrara, F. Mannucci, M. Pedani, M. Roca Sogorb, A supernova origin for dust in a high-redshift quasar. Nature 431, 533-535 (2004). astro-ph/0409577. https://doi.org/10.1038/nature02930

Y.N. Makurin, A.A. Sofronov, A.I. Gusev, A.L. Ivanovsky, Electronic structure and chemical stabilization of $\mathrm{C}_{28}$ fullerene. Chem. Phys. 270, 293-308 (2001). https://doi.org/10.1016/S0301-0104(01)00342-1 
S. Marassi, R. Schneider, M. Limongi, A. Chieffi, M. Bocchio, S. Bianchi, The metal and dust yields of the first massive stars. Mon. Not. R. Astron. Soc. 454, 4250-4266 (2015). 1509.08923. https://doi.org/ $10.1093 / \mathrm{mnras} / \mathrm{stv} 2267$

P.L. Marsden, F.C. Gillett, R.E. Jennings, J.P. Emerson, T. de Jong, F.M. Olnon, Far-infrared observations of the Crab Nebula. Astrophys. J. 278, L29 (1984)

P.G. Martin, Cosmic Dust. Its Impact on Astronomy. Oxford Studies in Physics (Clarendon, Oxford, 1978)

T. Matheson, A.V. Filippenko, R. Chornock, D.C. Leonard, W. Li, Helium emission lines in the type IC supernova 1999CQ. Astron. J. 119, 2303-2310 (2000a). astro-ph/0001270. https://doi.org/10.1086/301352

T. Matheson, A.V. Filippenko, L.C. Ho, A.J. Barth, D.C. Leonard, Detailed analysis of early to late-time spectra of supernova 1993J. Astron. J. 120, 1499-1515 (2000b). astro-ph/0006264. https://doi.org/ $10.1086 / 301519$

J.S. Mathis, W. Rumpl, K.H. Nordsieck, The size distribution of interstellar grains. Astrophys. J. 217, 425433 (1977). https://doi.org/10.1086/155591

M. Matsuura, Dust and molecular formation in supernovae, in Handbook of Supernovae, ed. by W.A. Athem, M. Paul (Springer, Berlin, 2017)

M. Matsuura, E. Dwek, M. Meixner, M. Otsuka, B. Babler, M.J. Barlow, J. Roman-Duval, C. Engelbracht, K. Sandstrom, M. Lakićević, J.T. van Loon, G. Sonneborn, G.C. Clayton, K.S. Long, P. Lundqvist, T. Nozawa, K.D. Gordon, S. Hony, P. Panuzzo, K. Okumura, K.A. Misselt, E. Montiel, M. Sauvage, Herschel detects a massive dust reservoir in supernova 1987A. Science 333, 1258 (2011). 1107.1477. https://doi.org/10.1126/science.1205983

M. Matsuura, E. Dwek, M.J. Barlow, B. Babler, M. Baes, M. Meixner, J. Cernicharo, G.C. Clayton, L. Dunne, C. Fransson, J. Fritz, W. Gear, H.L. Gomez née Morgan, M.A.T. Groenewegen, R. Indebetouw, R.J. Ivison, A. Jerkstrand, V. Lebouteiller, T.L. Lim, P. Lundqvist, C.P. Pearson, J. Roman-Duval, P. Royer, L. Staveley-Smith, B.M. Swinyard, P.A.M. van Hoof, J.T. van Loon, J. Verstappen, R. Wesson, G. Zanardo, J.A.D.L. Blommaert, L. Decin, W.T. Reach, G. Sonneborn, G.C. Van de Steene, J.A. Yates, A stubbornly large mass of cold dust in the ejecta of supernova 1987A. Astrophys. J. 800(1), 50 (2015)

M. Matsuura, R. Indebetouw, S. Woosley, V. Bujarrabal, F.J. Abellan, R. McCray, J. Kamenetzky, C. Fransson, M.J. Barlow, H.L. Gomez, P. Cigan, I. De Looze, J. Spyromilio, L. Staveley-Smith, G. Zanardo, P. Roche, J. Larsson, S. Viti, J.T. van Loon, J.C. Wheeler, M. Baes, R. Chevalier, P. Lundqvist, J.M. Marcaide, E. Dwek, M. Meixner, C.Y. Ng, G. Sonneborn, J. Yates, ALMA spectral survey of Supernova 1987A-molecular inventory, chemistry, dynamics and explosive nucleosynthesis. ArXiv e-prints 1704.02324 (2017)

S.M. Matz, G.H. Share, M.D. Leising, E.L. Chupp, W.T. Vestrand, Gamma-ray line emission from SN1987A. Nature 331, 416-418 (1988). https://doi.org/10.1038/331416a0

J.C. Mauerhan, N. Smith, J.M. Silverman, A.V. Filippenko, A.N. Morgan, S.B. Cenko, M. Ganeshalingam, K.I. Clubb, J.S. Bloom, T. Matheson, P. Milne, SN 2011ht: confirming a class of interacting supernovae with plateau light curves (type IIn-P). Mon. Not. R. Astron. Soc. 431, 2599-2611 (2013). 1209.0821. https://doi.org/10.1093/mnras/stt360

R. McCray, Supernova 1987A revisited. Annu. Rev. Astron. Astrophys. 31, 175-216 (1993). https://doi.org/ 10.1146/annurev.aa.31.090193.001135

W.P.S. Meikle, J. Spyromilio, G.F. Varani, D.A. Allen, Spectroscopy of supernova 1987A at 1-5 microns. I-The first year. Mon. Not. R. Astron. Soc. 238, 193-223 (1989)

W.P.S. Meikle, S. Mattila, A. Pastorello, C.L. Gerardy, R. Kotak, J. Sollerman, S.D. van Dyk, D. Farrah, A.V. Filippenko, P. Höflich, P. Lundqvist, M. Pozzo, J.C. Wheeler, A Spitzer space TelescopeStudy of SN 2003gd: still no direct evidence that core-collapse supernovae are major dust factories. Astrophys. J. 665(1), 608-617 (2007)

W.P.S. Meikle, R. Kotak, D. Farrah, S. Mattila, S.D. van Dyk, A.C. Andersen, R. Fesen, A.V. Filippenko, R.J. Foley, C. Fransson, C.L. Gerardy, P.A. Höflich, P. Lundqvist, M. Pozzo, J. Sollerman, J.C. Wheeler, Dust and the type II-Plateau supernova 2004dj. Astrophys. J. 732(2), 109 (2011)

M. Meixner, K.D. Gordon, R. Indebetouw, J.L. Hora, B.A. Whitney, R.D. Blum, W. Reach, J.P. Bernard, M. Meade, B. Babler, C.W. Engelbracht, B.Q. For, K. Misselt, U. Vijh, C. Leitherer, M. Cohen, E.B. Churchwell, F. Boulanger, J.A. Frogel, Y. Fukui, J.S. Gallagher III, V. Gorjian, J. Harris, D. Kelly, A. Kawamura, S. Kim, W.B. Latter, S.C. Madden, F. Kemper, A. Mizuno, N. Mizuno, J. Mould, A. Nota, M.S. Oey, K. Olsen, T. Onishi, R. Paladini, N. Panagia, P. Perez-Gonzalez, H. Shibai, S. Sato, L.J. Smith, L. Staveley-Smith, A.G.G.M. Tielens, T. Ueta, S.V. Dyk, K.M. Volk, M.W. Werner, D. Zaritsky, Spitzer survey of the large magellanic cloud: surveying the agents of a galaxy's evolution (SAGE). I. Overview and initial results. Astron. J. 132, 2268 (2006)

S. Miller, J. Tennyson, S. Lepp, A. Dalgarno, Identification of features due to $\mathrm{H} 3+$ in the infrared spectrum of supernova 1987A. Nature 355(6359), 420-422 (1992)

R. Minkowski, Spectra of supernovae. Publ. Astron. Soc. Pac. 53, 224 (1941). https://doi.org/10.1086/125315 
F.J. Molster, L.B.F.M. Waters, F. Kemper, The mineralogy of interstellar and circumstellar dust in galaxies, in Lecture Notes in Physics, vol. 815, ed. by T. Henning (Springer, Berlin, 2010), pp. 143-201

H. Mutschke, A.C. Andersen, D. Clément, T. Henning, G. Peiter, Infrared properties of SiC particles. Astron. Astrophys. 345, 187-202 (1999). astro-ph/9903031

L.R. Nittler, Presolar stardust in meteorites: recent advances and scientific frontiers. Earth Planet. Sci. Lett. 209, 259-273 (2003). https://doi.org/10.1016/S0012-821X(02)01153-6

L.R. Nittler, C.M.O. Alexander, J. Wang, X. Gao, Meteoritic oxide grain from supernova found. Nature 393, 222 (1998). https://doi.org/10.1038/30377

K.I. Nomoto, K. Iwamoto, T. Suzuki, The evolution and explosion of massive binary stars and type Ib-Ic-IIbIIL supernovae. Phys. Rep. 256, 173-191 (1995). https://doi.org/10.1016/0370-1573(94)00107-E

T. Nozawa, T. Kozasa, Formulation of non-steady-state dust formation process in astrophysical environments. Astrophys. J. 776, 24 (2013). 1308.1873. https://doi.org/10.1088/0004-637X/776/1/24

T. Nozawa, T. Kozasa, H. Umeda, K. Maeda, K. Nomoto, Dust in the early universe: dust formation in the ejecta of population III supernovae. Astrophys. J. 598, 785-803 (2003). astro-ph/0307108. https:// doi.org/10.1086/379011

T. Nozawa, T. Kozasa, N. Tominaga, I. Sakon, M. Tanaka, T. Suzuki, K. Nomoto, K. Maeda, H. Umeda, M. Limongi, T. Onaka, Early formation of dust in the ejecta of type Ib SN 2006jc and temperature and mass of the dust. Astrophys. J. 684(2), 1343-1350 (2008)

T. Nozawa, T. Kozasa, N. Tominaga, K. Maeda, H. Umeda, K. Nomoto, O. Krause, Formation and evolution of dust in type IIb supernovae with application to the Cassiopeia A supernova remnant. Astrophys. J. 713, 356-373 (2010). 0909.4145. https://doi.org/10.1088/0004-637X/713/1/356

T. Nozawa, K. Maeda, T. Kozasa, M. Tanaka, K. Nomoto, H. Umeda, Formation of dust in the ejecta of type Ia supernovae. Astrophys. J. 736, 45 (2011). 1105.0973. https://doi.org/10.1088/0004-637X/736/1/45

U. Ott, Presolar grains in meteorites: an overview and some implications. Planet. Space Sci. 49, 763-767 (2001). https://doi.org/10.1016/S0032-0633(01)00025-3

P.J. Owen, M.J. Barlow, The dust and gas content of the Crab Nebula. Astrophys. J. 801(2), 141 (2015)

Y.C. Pei, S.M. Fall, J. Bechtold, Confirmation of dust in damped Lyman-alpha systems. Astrophys. J. 378, 6-16 (1991). https://doi.org/10.1086/170401

M. Pettini, L.J. Smith, R.W. Hunstead, D.L. King, Metal enrichment, dust, and star formation in galaxies at high redshifts. 3: Zn and CR abundances for 17 damped Lyman-alpha systems. Astrophys. J. 426, 79-96 (1994). https://doi.org/10.1086/174041

D. Pinheiro Gonçalves, A. Noriega-Crespo, R. Paladini, P.G. Martin, S.J. Carey, The MIPSGAL view of supernova remnants in the galactic plane. Astron. J. 142(2), 47 (2011)

M. Pozzo, W.P.S. Meikle, J.T. Rayner, R.D. Joseph, A.V. Filippenko, R.J. Foley, W. Li, S. Mattila, J. Sollerman, Optical and infrared observations of the TypeIIP SN2002hh from days 3 to 397. Mon. Not. R. Astron. Soc. 368(3), 1169-1195 (2006)

D.M. Rank, P.A. Pinto, S.E. Woosley, J.D. Bregman, F.C. Witteborn, Nickel, argon and cobalt in the infrared spectrum of SN1987A - the core becomes visible. Nature 331(6156), 505-506 (1988)

T. Rauscher, A. Heger, R.D. Hoffman, S.E. Woosley, Nucleosynthesis in massive stars with improved nuclear and stellar physics. Astrophys. J. 576, 323-348 (2002). astro-ph/0112478. https://doi.org/10.1086/ 341728

W.T. Reach, J. Rho, A. Tappe, T.G. Pannuti, C.L. Brogan, E.B. Churchwell, M.R. Meade, B. Babler, R. Indebetouw, B.A. Whitney, A Spitzer space telescope infrared survey of supernova remnants in the inner galaxy. Astron. J. 131(3), 1479-1500 (2006)

A.C. Reber, S. Paranthaman, P.A. Clayborne et al., From SiO molecules to silicates in circumstellar space: atomic structures, growth patterns, and optical signatures of $\mathrm{Si}_{n} \mathrm{O}_{m}$ clusters. ACS Nano 2, 1729 (2008). https://doi.org/10.1021/nn7003958

J. Rho, T. Kozasa, W.T. Reach, J.D. Smith, L. Rudnick, T.A. DeLaney, J.A. Ennis, H.L. Gomez, A. Tappe, Freshly formed dust in the Cassiopeia A supernova remnant as revealed by the Spitzer space telescope. Astrophys. J. 673, 271 (2008)

J. Rho, T.H. Jarrett, W.T. Reach, H. Gomez, M. Andersen, Carbon monoxide in the Cassiopeia A supernova remnant. Astrophys. J. Lett. 693, L39-L43 (2009). 0901.2308. https://doi.org/10.1088/0004637X/693/1/L39

J. Rho, T. Onaka, J. Cami, W.T. Reach, Spectroscopic detection of carbon monoxide in the young supernova remnant Cassiopeia A. Astrophys. J. 747(1), L6 (2012)

P.F. Roche, D.K. Aitken, C.H. Smith, S.D. James, Old cold dust heated by supernova 1987A. Nature 337(6207), 533-535 (1989)

P.F. Roche, D.K. Aitken, C.H. Smith, Silicon monoxide in supernova 1987A. Mon. Not. R. Astron. Soc. 252, 39P-42P (1991)

J.M. Saken, R.A. Fesen, J.M. Shull, An IRAS survey of galactic supernova remnants. Astrophys. J. Suppl. Ser. 81, 715 (1992) 
K.M. Sandstrom, A.D. Bolatto, S. Stanimirović, J.T. van Loon, J.D.T. Smith, Measuring dust production in the small magellanic cloud core-collapse supernova remnant 1E 0102.2-7219. Astrophys. J. 696, 2138 (2009)

A. Sarangi, I. Cherchneff, The chemically controlled synthesis of dust in type II-P supernovae. Astrophys. J. 776, 107 (2013). 1309.5887. https://doi.org/10.1088/0004-637X/776/2/107

A. Sarangi, I. Cherchneff, Condensation of dust in the ejecta of type II-P supernovae. Astron. Astrophys. 575, A95 (2015). 1412.5522. https://doi.org/10.1051/0004-6361/201424969

P. Schilke, D.A. Neufeld, H.S.P. Müller, C. Comito, E.A. Bergin, D.C. Lis, M. Gerin, J.H. Black, M.G. Wolfire, N. Indriolo, J.C. Pearson, K.M. Menten, B. Winkel, Á. Sánchez Monge, T. Möller, B. Godard, E. Falgarone, Ubiquitous argonium $(\mathrm{ArH}+)$ in the diffuse interstellar medium: a molecular tracer of almost purely atomic gas. Astron. Astrophys. 566, A29 (2014)

E.M. Schlegel, A new subclass of type II supernovae? Mon. Not. R. Astron. Soc. 244, 269-271 (1990)

R. Schneider, A. Ferrara, R. Salvaterra, Dust formation in very massive primordial supernovae. Mon. Not. R. Astron. Soc. 351, 1379-1386 (2004). astro-ph/0307087. https://doi.org/10.1111/j.1365-2966. 2004.07876.x

J.Y. Seok, B.C.C. Koo, T. Onaka, Y. Ita, H.G. Lee, J.J. Lee, D.S. Moon, I. Sakon, H. Kaneda, H.M. Lee, M.G. Lee, S.E. Kim, Supernova remnants in the AKARI IRC survey of the large magellanic cloud. Publ. Astron. Soc. Jpn. 60, 453 (2008)

J.Y. Seok, B.C.C. Koo, T. Onaka, A survey of infrared supernova remnants in the large magellanic cloud. Astrophys. J. 779(2), 134 (2013)

J.Y. Seok, B.C.C. Koo, H. Hirashita, Dust cooling in supernova remnants in the large magellanic cloud. Astrophys. J. 807(1), 100 (2015)

T. Shigeyama, K. Nomoto, Theoretical light curve of SN 1987A and mixing of hydrogen and nickel in the ejecta. Astrophys. J. 360, 242-256 (1990). https://doi.org/10.1086/169114

B. Sibthorpe, P.A.R. Ade, J.J. Bock, E.L. Chapin, M.J. Devlin, S. Dicker, M. Griffin, J.O. Gundersen, M. Halpern, P.C. Hargrave, D.H. Hughes, W.S. Jeong, H. Kaneda, J. Klein, B.C. Koo, H.G. Lee, G. Marsden, P.G. Martin, P. Mauskopf, D.S. Moon, C.B. Netterfield, L. Olmi, E. Pascale, G. Patanchon, M. Rex, A. Roy, D. Scott, C. Semisch, M.D.P. Truch, C. Tucker, G.S. Tucker, M.P. Viero, D.V. Wiebe, AKARI and BLAST observations of the Cassiopeia A supernova remnant and surrounding interstellar medium. Astrophys. J. 719, 1553-1564 (2010). 0910.1094. https://doi.org/10.1088/0004-637X/719/2/1553

A. Sluder, M. Milosavljevic, M.H. Montgomery, Molecular nucleation theory of dust formation in corecollapse supernovae applied to SN 1987A. ArXiv e-prints. 1612.09013 (2016)

N. Smith, R. Chornock, W. Li, M. Ganeshalingam, J.M. Silverman, R.J. Foley, A.V. Filippenko, A.J. Barth, SN 2006tf: precursor eruptions and the optically thick regime of extremely luminous type IIn supernovae. Astrophys. J. 686, 467-484 (2008). 0804.0042. https://doi.org/10.1086/591021

N. Smith, J.M. Silverman, A.V. Filippenko, M.C. Cooper, T. Matheson, F. Bian, B.J. Weiner, J.M. Comerford, Systematic blueshift of line profiles in the type IIn supernova 2010j1: evidence for post-shock dust formation? Astron. J. 143, 17-20 (2012). 1108.2869. https://doi.org/10.1088/0004-6256/143/1/17

J. Spyromilio, B. Leibundgut, Carbon monoxide in supernova 1995ad. Mon. Not. R. Astron. Soc. 283, L89L93 (1996)

J. Spyromilio, W.P.S. Meikle, R.C.M. Learner, D.A. Allen, Carbon monoxide in supernova 1987A. Nature 334(6180), 327-329 (1988)

J. Spyromilio, B. Leibundgut, R. Gilmozzi, Carbon monoxide in type II supernovae. Astron. Astrophys. 376(1), 188-193 (2001)

S. Stanimirović, A.D. Bolatto, K.M. Sandstrom, A.K. Leroy, J.D. Simon, B.M. Gaensler, R.Y. Shah, J.M. Jackson, Spitzer space telescope detection of the young supernova remnant 1E 0102.2-7219. Astrophys. J. 632(2), L103-L106 (2005)

B.E.K. Sugerman, B. Ercolano, M.J. Barlow, A.G.G.M. Tielens, G.C. Clayton, A.A. Zijlstra, M. Meixner, A. Speck, T.M. Gledhill, N. Panagia, M. Cohen, K.D. Gordon, M. Meyer, J. Fabbri, J.E. Bowey, D.L. Welch, M.W. Regan, R.C. Kennicutt, Massive-star supernovae as major dust factories. Science 313, 196-200 (2006). astro-ph/0606132. https://doi.org/10.1126/science.1128131

N.B. Suntzeff, P. Bouchet, The bolometric light curve of SN 1987A. I-results from ESO and CTIO U to Q0 photometry. Astron. J. 99, 650-663 (1990)

H. Suto, C. Koike, H. Sogawa, A. Tsuchiyama, H. Chihara, K. Mizutani, Infrared spectra of fayalite crystal. Astron. Astrophys. 389, 568-571 (2002). https://doi.org/10.1051/0004-6361:20020501

R.A. Syunyaev, A. Kaniovskii, V. Efremov, M. Gilfanov, E. Dhurazov, S.A. Grebenev, A.V. Kuznetsov, A.S. Melioranskii, N.S. Yamburenko, S. Yunin, D. Stepanov, I. Chulkov, N. Pappe, M.N. Boyarskii, E.A. Gavrilova, V.M. Loznikov, A. Prudkoglyad, V.G. Rodin, C. Reppin, W. Pietsch, J. Engelhauser, J. Trumper, W. Voges, E. Kendziorra, M. Bezler, R. Staubert, A.C. Brinkman, J. Heise, W.A. Mels, R. Jager, G.K. Skinner, O. Al-Emam, T.G. Patterson, A.P. Willmore, Detection of hard X-rays from supernova 1987A—preliminary Mir-Kvant results. Sov. Astron. Lett. 13, 431 (1987) 
T. Szalai, J. Vinkó, Twelve type II-P supernovae seen with the eyes of Spitzer. Astron. Astrophys. 549, A79 (2013). 1211.0854. https://doi.org/10.1051/0004-6361/201220015

T. Szalai, J. Vinkó, Z. Balog, A. Gáspár, M. Block, L.L. Kiss, Dust formation in the ejecta of the type II-P supernova 2004dj. Astron. Astrophys. 527, A61 (2011). 1012.2035. https://doi.org/10.1051/ 0004-6361/201015624

T. Szalai, J. Vinkó, A.P. Nagy, J.M. Silverman, J.C. Wheeler, G. Dhungana, G.H. Marion, R. Kehoe, O.D. Fox, K. Sárneczky, G. Marschalkó, B.I. Bíró, T. Borkovits, T. Hegedüs, R. Szakáts, F.V. Ferrante, E. Bányai, G. Hodosán, J. Kelemen, A. Pál, The continuing story of SN IIb 2013df: new optical and IR observations and analysis. Mon. Not. R. Astron. Soc. 460(2), 1500-1518 (2016)

T. Temim, E. Dwek, The importance of physical models for deriving dust masses and grain size distributions in supernova ejecta. I. Radiatively heated dust in the Crab Nebula. Astrophys. J. 774, 8 (2013). 1302.5452. https://doi.org/10.1088/0004-637X/774/1/8

T. Temim, R.D. Gehrz, C.E. Woodward, T.L. Roellig, N. Smith, L. Rudnick, E.F. Polomski, K. Davidson, L. Yuen, T. Onaka, Spitzer space TelescopeInfrared imaging and spectroscopy of the Crab Nebula. Astron. J. 132(4), 1610-1623 (2006)

T. Temim, E. Dwek, R.G. Arendt, K.J. Borkowski, S.P. Reynolds, P. Slane, J.D. Gelfand, J.C. Raymond, A massive shell of supernova-formed dust in SNR G54.1+0.3. Astrophys. J. 836(1), 129 (2017)

F.K. Thielemann, Nucleosynthesis in supernovae, hypernovae/gamma-ray bursts and compact binary mergers, in 14th International Symposium on Nuclei in the Cosmos (NIC2016), ed. by S. Kubono, T. Kajino, S. Nishimura, T. Isobe, S. Nagataki, T. Shima, Y. Takeda (2017), p. 010605. https://doi.org/ 10.7566/JPSCP.14.010605

F.K. Thielemann, K. Nomoto, M.A. Hashimoto, Core-collapse supernovae and their ejecta. Astrophys. J. 460, 408 (1996). https://doi.org/10.1086/176980

F.K. Thielemann, R. Hirschi, M. Liebendörfer, R. Diehl, Massive stars and their supernovae, in Lecture Notes in Physics, vol. 812, ed. by R. Diehl, D.H. Hartmann, N. Prantzos (Springer, Berlin, 2011), pp. 153-232. 1008.2144

P. Todini, A. Ferrara, Dust formation in primordial type II supernovae. Mon. Not. R. Astron. Soc. 325, 726736 (2001). astro-ph/0009176. https://doi.org/10.1046/j.1365-8711.2001.04486.x

C. Travaglio, R. Gallino, S. Amari, E. Zinner, S. Woosley, R.S. Lewis, Low-density graphite grains and mixing in type II supernovae. Astrophys. J. 510, 325-354 (1999). https://doi.org/10.1086/306551

R.J. Trumpler, Absorption of light in the galactic system. Publ. Astron. Soc. Pac. 42, 214 (1930). https:// doi.org/10.1086/124039

M. Turatto, Classification of supernovae, in Supernovae and Gamma-Ray Bursters, ed. by K. Weiler. Lecture Notes in Physics, vol. 598 (Springer, Berlin, 2003), pp. 21-36. astro-ph/0301107

H. Umeda, K. Nomoto, Nucleosynthesis of zinc and iron peak elements in population III type II supernovae: comparison with abundances of very metal poor halo stars. Astrophys. J. 565, 385-404 (2002). astro-ph/0103241. https://doi.org/10.1086/323946

V.P. Utrobin, N.N. Chugai, A.A. Andronova, Asymmetry of SN 1987A: fast Ni-56 clump. Astron. Astrophys. 295, 129-135 (1995)

S.D. van Dyk, Late-time dust emission from the type IIn supernova 1995N. Astron. J. 145(5), 118 (2013)

H.J. Walker, A brief history of infrared astronomy. Astron. Geophys. 41(5), 10 (2000). https://doi.org/ 10.1046/j.1468-4004.2000.41510.x

S.H.J. Wallström, C. Biscaro, F. Salgado, J.H. Black, I. Cherchneff, S. Muller, O. Berné, J. Rho, A.G.G.M. Tielens, CO rotational line emission from a dense knot in Cassiopeia A. Astron. Astrophys. 558, L2 (2013)

R. Wesson, M.J. Barlow, M. Matsuura, B. Ercolano, The timing and location of dust formation in the remnant of SN 1987A. Mon. Not. R. Astron. Soc. 446(2), 2089-2101 (2014)

J.C. Wheeler, S. Benetti Supernovae, Cox, A.N., p. 451 (2000)

P.A. Whitelock, R.M. Catchpole, J.W. Menzies, M.W. Feast, S.E. Woosley, D. Allen, F. van Wyk, F. Marang, C.D. Laney, H. Winkler, K. Sekiguchi, L.A. Balona, B.S. Carter, J.H. Spencer Jones, J.D. Laing, T.L. Evans, A.P. Fairall, D.A.H. Buckley, I.S. Glass, M.V. Penston, L.N. da Costa, S.A. Bell, C. Hellier, M. Shara, A.F.J. Moffat, Spectroscopic and photometric observations of SN1987A. VI-days 617 to 792. R. Astron. Soc. 240, 7 (1989)

D.C.B. Whittet (ed.), Dust in the galactic environment (2003)

B.J. Williams, K.J. Borkowski, S.P. Reynolds, W.P. Blair, P. Ghavamian, S.P. Hendrick, K.S. Long, S. Points, J.C. Raymond, R. Sankrit, R.C. Smith, P.F. Winkler, Dust destruction in fast shocks of core-collapse supernova remnants in the large magellanic cloud. Astrophys. J. 652(1), L33-L36 (2006)

A. Wongwathanarat, E. Müller, H.T. Janka, Three-dimensional simulations of core-collapse supernovae: from shock revival to shock breakout. Astron. Astrophys. 577, A48 (2015). 1409.5431. https://doi.org/ 10.1051/0004-6361/201425025 
D.H. Wooden, D.M. Rank, J.D. Bregman, F.C. Witteborn, A.G.G.M. Tielens, M. Cohen, P.A. Pinto, T.S. Axelrod, Airborne spectrophotometry of SN 1987A from 1.7 to 12.6 microns-time history of the dust continuum and line emission. Astrophys. J. Suppl. Ser. 88, 477-507 (1993). https://doi.org/10.1086/191830

S.E. Woosley, SN 1987A-after the peak. Astrophys. J. 330, 218-253 (1988). https://doi.org/10.1086/ 166468

S.E. Woosley, T.A. Weaver, The evolution and explosion of massive stars. II. Explosive hydrodynamics and nucleosynthesis. Astrophys. J. Suppl. 101, 181 (1995). https://doi.org/10.1086/192237

S.E. Woosley, D. Hartmann, P.A. Pinto, Hard emission at late times from SN 1987A. Astrophys. J. 346, 395-404 (1989). https://doi.org/10.1086/168019

M.R. Zachariah, W. Tsang, Application of ab-initio molecular orbital and reaction rate theories to nucleation kinetics. Aerosol Sci. Technol. 19, 499 (1993)

G. Zanardo, L. Staveley-Smith, R. Indebetouw, R.A. Chevalier, M. Matsuura, B.M. Gaensler, M.J. Barlow, C. Fransson, R.N. Manchester, M. Baes, J.R. Kamenetzky, M. Lakićević, P. Lundqvist, J.M. Marcaide, I. Marti-Vidal, M. Meixner, C.Y. Ng, S. Park, G. Sonneborn, J. Spyromilio, J.T. van Loon, Spectral and morphological analysis of the remnant of supernova 1987A with ALMA and ATCA. Astrophys. J. 796(2), 82 (2014)

E. Zinner, F. Gyngard, Y. Lin, Silicon carbide grains of type X and supernova nucleosynthesis, in Nuclei in the Cosmos (2010), p. 148 